 Physical Layer Security in the Face of CSI Feedback Delays
}

\author{
Lei Wang, Student Member, IEEE, Yueming Cai, Senior Member, IEEE, Yulong Zou, Senior Member, IEEE, \\ Weiwei Yang, Member, IEEE, and Lajos Hanzo, Fellow, IEEE
}

6 Abstract-We enhance the physical layer security (PLS) of 7 amplify-and-forward (AF) relaying networks with the aid of joint 8 relay and jammer selection (JRJS), despite the deleterious effect 9 of channel state information (CSI) feedback delays. Furthermore, 10 we conceive a new outage-based characterization approach for the 11 JRJS scheme. The traditional best relay selection (TBRS) is also 12 considered as a benchmark. We first derive closed-form ex13 pressions of both the connection outage probability (COP) and 14 the secrecy outage probability (SOP) for both the TBRS and 15 JRJS schemes. Then, a reliable and secure connection probability 16 (RSCP) is defined and analyzed for characterizing the effect of 17 the correlation between the COP and the SOP introduced by the 18 corporate source-relay link. The reliability-security ratio (RSR) 19 is introduced for characterizing the relationship between the re20 liability and the security through asymptotic analysis. Moreover, 21 the concept of effective secrecy throughput is defined as the 22 product of the secrecy rate and of the RSCP for the sake of 23 characterizing the overall efficiency of the system, as determined 24 by the transmit SNR, the secrecy codeword rate, and the power 25 sharing ratio between the relay and the jammer. The impact of 26 the direct source-eavesdropper link and additional performance 27 comparisons with respect to other related selection schemes are 28 also included. Our numerical results show that the JRJS scheme 29 outperforms the TBRS method both in terms of the RSCP and in 30 terms of its effective secrecy throughput, but it is more sensitive to 31 the feedback delays. Increasing the transmit signal-to-noise ratio 32 (SNR) will not always improve the overall throughput. Moreover, 33 the RSR results demonstrate that, upon reducing the CSI feedback 34 delays, the reliability improves more substantially than the secu35 rity degrades, implying an overall improvement in terms of the 36 security-reliability tradeoff. Additionally, the secrecy throughput 37 loss due to the second-hop feedback delay is more pronounced 38 than that due to the first-hop one.

Manuscript received October 2, 2014; revised February 25, 2015 and July 27, 2015; accepted September 8, 2015. This work was supported in part by the National Natural Science Foundation of China under Grant 61371122, Grant 61471393, and Grant 61501512 and in part by the Natural Science Foundation of Jiangsu Province under Grant BK20150718 and Grant BK20150040. The review of this paper was coordinated by Prof. M. C. Gursoy.

L. Wang, Y. Cai, and W. Yang are with the College of Communications Engineering, PLA University of Science and Technology, Nanjing 210007, China (e-mail: csu-wl@163.com; caiym@vip.sina.com; yww_1010@aliyun.com).

Y. Zou is with the School of Telecommunications and Information Engineering, Nanjing University of Posts and Telecommunications, Nanjing 210003, China (e-mail: yulong.zou@njupt.edu.cn).

L. Hanzo is with the Department of Electronics and Computer Science, University of Southampton, Southampton SO17 1BJ, U.K. (e-mail: 1h@ecs. soton.ac.uk).

Color versions of one or more of the figures in this paper are available online at http://ieeexplore.ieee.org.

Digital Object Identifier 10.1109/TVT.2015.2478029
Index Terms-Effective secrecy throughput, feedback delay, 39 physical layer security (PLS), relay and jammer selection, 40 reliability and security.

\section{INTRODUCTION}

$\mathbf{W}$ IRELESS communications systems are particularly 43 vulnerable to security attacks because of the inherent 45 openness of the transmission medium. Traditionally, the infor- 46 mation privacy of wireless networks has been focused on the 47 higher layers of the protocol stack employing cryptographically 48 secure schemes. However, these methods typically assume a 49 limited computing power for the eavesdroppers and exhibit 50 inherent vulnerabilities in terms of the inevitable secret key 51 distribution and management [1]. In recent years, physical 52 layer security (PLS) has emerged as a promising technique of 53 improving the confidentiality wireless communications, which 54 exploits the time-varying properties of fading channels, instead 55 of relying on conventional cryptosystems. The pivotal idea of 56 PLS solutions is to exploit the dynamically fluctuating random 57 nature of radio channels for maximizing the uncertainty con- 58 cerning the source messages at the eavesdropper [2], [3].

59

To achieve this target, several PLS-enhancement approaches 60 have been proposed in the literature, including secrecy- 61 enhancing channel coding [4], secure on-off transmission de- 62 signs [5], secrecy-improving beamforming (BF)/precoding, and 63 artificial-noise-aided techniques relying on multiple antennas 64 [6], as well as secure relay-assisted transmission techniques [7]. 65 Specifically, apart from improving the reliability and coverage 66 of wireless transmissions, user cooperation also has a great 67 potential in terms of enhancing the wireless security against 68 eavesdropping attacks. There has been a growing interest in 69 improving the security of cooperative networks at the physical 70 layer [8]-[14]. To explore the spatial diversity potential of the 71 relaying networks and to boost the secrecy capacity (the differ- 72 ence between the channel capacity of the legitimate main link 73 and that of the eavesdropping link), most of the existing work 74 has been focused on secrecy-enhancing BF [8], [9], as well as 75 on intelligent relay node/jammer node (RN/JN) selection, etc. 76 Notably, given the availability of multiple relays, appropriately 77 designed RN/JN selection is capable of achieving a signifi- 78 cant security improvement for cooperative networks, which is 79 emerging as a promising research topic. In particular, Zou et al. 80 investigated both amplify-and-forward (AF)- and decode-and- 81 forward (DF)-based optimal relay selection conceived for 82 
83 enhancing the PLS in cooperative wireless networks [10], [11], 84 where the global channel state information (CSI) of both the 85 main link and the eavesdropping link was assumed to be avail86 able. Similarly, jamming techniques, which impose artificial 87 interference on the eavesdropper, have also attracted substantial 88 attention [12]-[14]. More specifically, several sophisticated 89 joint relay and jammer selection (JRJS) schemes were proposed 90 in [12], where the beneficially selected relay increases the reli91 ability of the main link, whereas the carefully selected jammer 92 imposes interference on the eavesdropper and simultaneously 93 protects the legitimate destination from interference. In [13] 94 and [14], cooperative jamming has been studied in the context 95 of bidirectional scenarios, and efficient RN/JN selection criteria 96 have been developed for achieving improved secrecy rates with 97 the aid of multiple relays. Furthermore, more effective relaying 98 and jamming schemes, when taking the information leakage 99 of the source-eavesdropper link into consideration, have been 100 presented lately in [15] and [16].

101 Nevertheless, an idealized assumption of the previously re102 ported research on PLS is the availability of perfect channel 103 state information (CSI), which is regarded as a stumbling block 104 in the way of invoking practical secrecy-enhancing Wyner 105 coding, on-off design, BF/precoding, and $\mathrm{RN} / \mathrm{JN}$ selection. 106 However, this idealized simplifying assumption is not realistic, 107 since practical channel estimation imposes CSI imperfections, 108 which are aggravated by the feedback delay, limited-rate feed109 back, and channel estimation errors (CEEs) [17]. Generally, the 110 related research has been focused on the issues of robust secure $111 \mathrm{BF}$ design from an average secrecy-rate-based optimization 112 perspective for point-to-point multiantenna aided channels and 113 relay channels [18], [19] supporting delay-tolerant systems. 114 For systems imposing stringent delay constraints, particularly 115 in imperfect CSI scenarios, perfect secrecy cannot always be 116 achieved. Hence, the secrecy-outage-based characterization of 117 systems is more appropriate, which provides a probabilistic 118 performance measure of secure communication. The concept 119 of secrecy outage was adopted in [20] for characterizing the 120 probability of having both reliable and secure transmission, 121 which, however, is inapplicable for the imperfect CSI case and 122 fails to distinguish a connection outage from the secrecy outage. 123 In [21], an alternative secrecy outage formulation is proposed 124 for characterizing the attainable security level and provided 125 a general framework for designing transmission schemes that 126 meet specific target security requirements. To quantify both the 127 reliability and security performance at both the legitimate and 128 eavesdropper nodes separately, two types of outages, namely, 129 the connection outage probability (COP) and the secrecy outage 130 probability (SOP) are introduced. Then, considering the impact 131 of time delay caused by the antenna selection process at the 132 legitimate receiver, $\mathrm{Hu}$ et al. [22] proposed a new secure 133 transmission scheme in the multiinput multioutput multieaves134 dropper wiretap channel. Much recently, considering the out135 dated CSI from the legitimate receiver, a new secure on-off 136 transmission scheme was proposed for enhancing the secrecy 137 throughput in [23].

138 Moreover, prior studies of the outage-based secure trans139 mission design are limited to single-antenna-assisted single140 hop systems and have not been considered for cooperative relaying systems. Hence, the issues of secure transmissions 141 over cooperative relaying channels expressed in terms of the 142 SOP, COP, and secrecy throughput constitute an open problem. 143 On the other hand, apart from CEE, the CSI feedback delay 144 results in critical challenges for the PLS of cooperative relaying 145 systems, particularly when considering the specifics of RN/JN 146 selection. In [15], the effects of outdated CSI knowledge con- 147 cerning the legitimate links on the ergodic secrecy rate achieved 148 by the proposed secure transmission strategy in the context 149 of DF relaying is investigated. The impact of CSI feedback 150 delay on the secure relay and jammer selection conceived for 151 DF relaying was investigated in [24], albeit only in terms 152 of the SOP. In our previous study [25], we considered the 153 secure transmission design and the secrecy performance of an 154 opportunistic DF system relying on outdated CSI, where only a 155 single relay is invoked. Additionally, during the revision of this 156 work, we investigated the security performance for outdated AF 157 relay selection in [26]. Therefore, in this treatise, we extend 158 our investigations to the PLS of multiple AF relaying assisted 159 networks relying on RN/JN selection.

Explicitly, we focus our attention on the outage-based char- 161 acterization of secure transmissions in cooperative relay-aided 162 networks relying on realistic CSI feedback delay. To exploit the 163 multirelay induced diversity gain and the associated jamming 164 capabilities, joint AF relay node and jammer node selection 165 is employed by the relay-destination link. We assume that, in 166 line with the practical reality, the instantaneous eavesdropper's 167 CSI is unavailable at the legitimate transmitter and that the 168 RN/JN selections are performed based on the outdated CSI of 169 the main links. Two types of cooperative strategies are invoked 170 by our cooperative network operating under secrecy constraints, 171 namely, the traditional best relay selection (TBRS) strategy and 172 the JRJS strategy. Specifically, the main contributions of this 173 paper can be summarized as follows.

- We develop an outage-based characterization for quan- 176 tifying both the reliability and security performance of 177 a two-hop AF relaying system. Specifically, in contrast 178 to [21] and [22], we propose the novel definition of 179 the reliable and secure connection probability (RSCP). 180 Explicitly, closed-form expressions of the COP, the SOP, 181 and the RSCP are derived for both the TBRS and for our 182 JRJS strategies. Numerical results demonstrate that the 183 JRJS scheme outperforms the TBRS scheme in terms of 184 its RSCP.

- We also introduce the reliability-security ratio (RSR) 186 for characterizing their direct relationship by a single 187 parameter through the asymptotic analysis of the COP and 188 the SOP in the high-SNR regime. We derive the RSR for 189 both the TBRS and JRJS strategies for investigating the 190 effect of secrecy codeword rate setting, as well as that 191 of the feedback delay and that of the power sharing ratio 192 between the relay and the jammer on the RSR.

- We then modify the definition of effective secrecy 194 throughput by multiplying the secrecy rate with the RSCP, 195 which results in an optimization problem of the trans- 196 mit signal-to-noise ratio (SNR), secrecy codeword rate, 197 and power sharing between the relay and the jammer. 198 


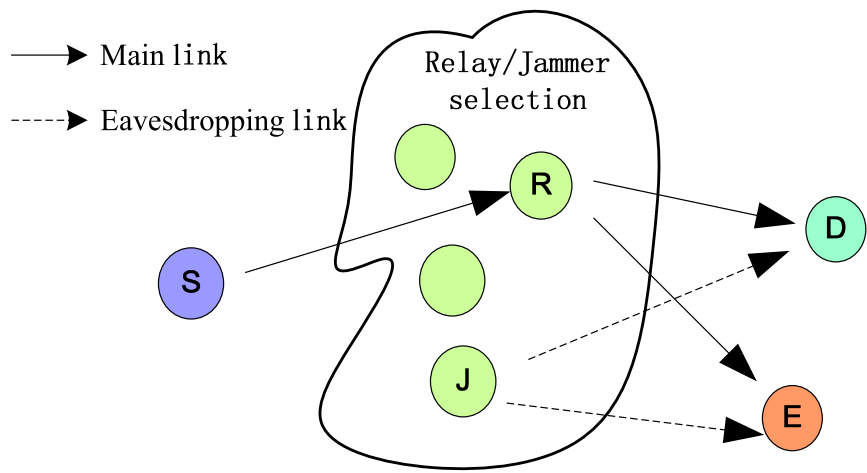

Fig. 1. Cooperative relaying network assisted by multiple relays in the presence of an eavesdropper.

206 The remainder of this paper is organized as follows. 207 Section II introduces our system model and describes both 208 the TBRS and our JRJS strategies. In Sections III and IV, 209 we present the mathematical framework of our performance 210 analysis both for the TBRS strategy and for the JRJS strategy, 211 respectively, including the COP, the SOP, the RSCP, the RSR, 212 and the effective secrecy throughput. Our numerical results 213 and discussions are provided in Section V. Finally, Section VI 214 presents our concluding remarks.

\section{SySTEM MODEL}

\section{A. System Description}

217 Consider a cooperative relaying network consisting of a 218 source $S$, a destination $D, K_{r}$ relays $R_{k}, k=1, \ldots, K_{r}$, and 219 an eavesdropper $E$, as shown in Fig. 1, where all nodes are 220 equipped with a single transmit antenna (TA), except for the 221 source, which has $N_{t}$ TAs. The cooperative relay architecture 222 in Fig. 1 is generally applicable to diverse practical wireless 223 systems in the presence of an eavesdropper, including the 224 family of wireless sensor networks (WSNs), mobile ad hoc 225 networks (MANETs), and the long-term evolution advanced 226 cellular systems [11].

227 To exploit the diversity potential of multiple relay nodes over 228 independently fading channels, AF relay/jammer selection is 229 employed. All relays operate in the half-duplex AF mode, and 230 data transmission is performed in two phases. More particu231 larly, during the broadcast phase, the source node transmits its 232 signal to a selected relay with the aid of BF, which is invoked 233 for forwarding the signal received from $S$ to $D$. An inherent 234 assumption is that the transmit BF weights are based on the 235 CSI estimates quantified and fed back by the selected relay. 236 During the cooperative phase, a pair of appropriately selected 237 relays transmit toward $D$ and $E$, respectively. A conventional relay (denoted by $R^{*}$ ) forwards the source's message to the 238 destination. Another relay (denoted by $J^{*}$ ) operates in the 239 "jammer mode" and imposes intentional interference upon $E$ in 240 order confuse it. However, $D$ is unable to mitigate the artificial 241 interference emanating from the jammer node $J^{*}$ due to its 242 critical secrecy constraints [12]. It should be noted that both 243 the process of RN/JN selection and the feedback of the transmit 244 BF weights from $R^{*}$ to $S$ may impose a time lag between the 245 data transmission and the channel estimation. These time delays 246 are denoted by $T_{d_{S R}}$ and $T_{d_{R D}}$, respectively. Furthermore, we 247 assume that the $\mathrm{BF}$ and $\mathrm{RN} / \mathrm{JN}$ selection process is based 248 on the perfectly estimated but outdated CSI. We employ the 249 first-order autoregressive outdated CSI model of [20], while 250 relying on the correlation coefficients of $\rho_{S R}=J_{0}\left(2 \pi f_{d} T_{d_{S R}}\right) 251$ and $\rho_{R D}=J_{0}\left(2 \pi f_{d} T_{d_{R D}}\right)$ for the two hops, where $J_{0}(\cdot)$ is 252 the zero-order Bessel function of the first kind, and $f_{d}$ is the 253 Doppler frequency.

A slow flat block Rayleigh fading environment is assumed, 255 where the channel remains static for the coherence interval (one 256 slot) and changes independently in different coherence inter- 257 vals, as denoted by $h_{i, j} \sim \mathcal{C N}\left(0, \sigma_{i, j}^{2}\right), i, j \in\{S, R, J, D, E\} .258$ The direct communication links are assumed to be unavailable 259 due to the presence of obstructions between $S$ and $D$, as well 260 as the eavesdropper. ${ }^{1}$ This assumption follows the rationale of 261 [12] and has been routinely exploited in previous literature (see 262 [27] and [28] and the references therein), where the source 263 and relays belong to the same cluster, whereas the destination 264 and the eavesdropper are located in another. More specifically, 265 this assumption is particularly valid in networks with broadcast 266 and unicast transmission, where each terminal is a legitimate 267 receiver for one signal and acts as an eavesdropper for some 268 other signal. Therefore, the security concerns are only related 269 to the cooperative relay-aided channel. Furthermore, additive 270 white Gaussian noise (AWGN) is assumed with zero mean 271 and unit variance $N_{0}$. Let $P_{i}$ be the transmit power of node 272 $i$, and the instantaneous SNR of the $i \rightarrow j$ link is given by 273 $\gamma_{i, j}=P_{i}\left|h_{i, j}\right|^{2} / N_{0}$.

We employ the constant-rate Wyner coding scheme for con- 275 structing wiretap codes of [2] to meet the PLS requirements 276 due to the fact that the accurate global CSI is not available. 277 Let $\mathbb{C}\left(R_{0}, R_{s}, N\right)$ denote the set of all possible Wyner codes 278 of length $N$, where $R_{0}$ is the codeword transmission rate, and 279 $R_{s}$ is the confidential information rate $\left(R_{0}>R_{s}\right)$. The positive 280 rate difference $R_{e}=R_{0}-R_{s}$ is the cost of providing secrecy 281 against the eavesdropper. A confidential message is encoded 282 into a codeword at $S$ and then transmitted to $D$.

\section{B. Secure Transmission}

In the broadcast phase, $S$ transmits its BF signal $s(t)$ to the 285 selected relay $R^{*}$, where the relay selection is performed 286 before data transmission commences, and the selection cri- 287 terion will be detailed later in the context of the cooper- 288 ative phase. The transmit BF vector $\mathbf{w}\left(t \mid T_{d}\right)$ is calculated 289 using the perfectly estimated but outdated CSI given by 290

\footnotetext{
${ }^{1}$ The case when the $S \rightarrow E$ link is introduced will be investigated separately in Section VI.
} 
$291 \mathbf{w}\left(t \mid T_{d_{S R}}\right)=\mathbf{h}_{S R^{*}}^{H}\left(t-T_{d}\right) /\left|\mathbf{h}_{S R^{*}}\left(t-T_{d_{S R}}\right)\right|$ [29], where we 292 have $\mathbf{h}_{S R^{*}}(t)=\left[h_{S R^{*}, 1}(t), \ldots, h_{S R^{*}, N_{t}}(t)\right]^{T}$, and the signal 293 received by the relay $R^{*}$ can be written as

$$
y_{R^{*}}(t)=\sqrt{P_{s}} \mathbf{w}\left(t \mid T_{d}\right) \mathbf{h}_{S R^{*}}(t) s(t)+n_{S R^{*}}(t)
$$

294 where $n_{S R^{*}}(t)$ is the AWGN at the relay. Then, we can 295 define the received SNR at the relay node as $\gamma_{S R}=$ $296 P_{S}\left|\mathbf{w}\left(t \mid T_{d_{S R}}\right) \mathbf{h}_{S R^{*}}(t)\right|^{2} / N_{0}$.

297 In the cooperative phase, we consider two RN/JN selection 298 schemes performed by $D$ : relay selection without jamming 299 and JRJS.

300 1) Traditional Best Relay Selection: The first category of so301 lutions does not involve a jamming process, and therefore, only 302 a conventional relay accesses the channel during the second 303 phase of the protocol. The relay selection process is performed 304 based on the highest instantaneous SNR of the second hop, 305 which is formulated as

$$
R^{*}=\arg \max _{R_{k} \in \mathcal{R}}\left\{\frac{\tilde{\gamma}_{R_{k} D}}{\mathbb{E}\left[\gamma_{R_{k} E}\right]}=\frac{P_{R}\left|\tilde{h}_{R_{k} D}\left(t-T_{d}\right)\right|^{2}}{N_{0} \mathbb{E}\left[\gamma_{R_{k} E}\right]}\right\}
$$

306 where $\tilde{\gamma}_{R_{k} D}$ is the instantaneous SNR in the relay selection 307 process, and $\mathbb{E}\left[\gamma_{R_{k} E}\right]$ denotes the average SNR at $E$. We can AQ1 308 model $\gamma_{R_{k} D}$ and $\tilde{\gamma}_{R_{k} D}$ as two gamma distributed random 309 variables having the correlation factor of $\rho_{R D}^{2}$.

310 During the second phase, the received signal $y_{R^{*}}(t)$ 311 is multiplied by a time-variant AF-relay gain $G$ and 312 retransmitted to $D$, where we have $G=$ $313 \sqrt{P_{R} /\left(P_{S}\left|\mathbf{w}_{\text {opt }}\left(t \mid T_{d_{S R}}\right) \mathbf{h}_{S R^{*}}(t)\right|^{2}+N_{0}\right)}$. After further math314 ematical manipulations, the mutual information (MI) between $315 S$ and $D$, as well as the eavesdropper, can be written as

$$
\begin{aligned}
& I_{D}^{\mathrm{TBRS}}=\frac{1}{2} \log \left(1+\gamma_{D}^{\mathrm{TBRS}}\right)=\frac{1}{2} \log \left(1+\frac{\gamma_{S R} \gamma_{R^{*} D}}{\gamma_{S R}+\gamma_{R^{*} D}+1}\right) \\
& I_{E}^{\mathrm{TBRS}}=\frac{1}{2} \log \left(1+\gamma_{E}^{\mathrm{TBRS}}\right)=\frac{1}{2} \log \left(1+\frac{\gamma_{S R} \gamma_{R^{*} E}}{\gamma_{S R}+\gamma_{R^{*} E}+1}\right) .
\end{aligned}
$$

316 2) Joint Relay and Jammer Selection: Similarly, consider317 ing the unavailability of the instantaneous CSI regarding the 318 eavesdropper, we adopt a suboptimal RN/JN selection metric 319 conditioned on the outdated CSI as

$$
\begin{aligned}
R^{*} & =\arg \max _{R_{k} \in \mathcal{R}}\left\{\frac{\tilde{\gamma}_{R_{k} D}}{\mathbb{E}\left[\gamma_{R_{k} E}\right]}\right\} \\
J^{*} & =\arg \min _{R_{k} \in \mathcal{R}-R^{*}}\left\{\frac{\tilde{\gamma}_{R_{k} D}}{\mathbb{E}\left[\gamma_{R_{k} E}\right]}\right\}
\end{aligned}
$$

320 where $J^{*}$ is selected for minimizing the interference imposed 321 on $D$.

322 It should be noted that, to have the same transmit power as 323 that of the TBRS case, we assume that $P_{R *}+P_{J^{*}}=P_{R}$ for 324 our JRJS strategy and introduce $\lambda=P_{R^{*}} /\left(P_{R^{*}}+P_{J^{*}}\right)$ as the ratio of the relay's transmit power to the total power required 325 by the active relay and jammer.

In the cooperative phase, $R^{*}$ will also amplify the received 327 signal $y_{R^{*}}(t)$ by $G$ and forward it to $D$. At the same time, the 328 jammer $J^{*}$ will generate intentional interference to confuse $E, 329$ which will also cause interference at $D$. Consequently, the MI 330 between the terminals is given by

$$
I_{D}^{\mathrm{JRJS}}=\frac{1}{2} \log \left(1+\gamma_{D}^{\mathrm{JRJS}}\right)=\frac{1}{2} \log \left(1+\frac{\gamma_{S R} \frac{\gamma_{R^{*} D}}{\gamma_{J^{*} D}+1}}{\gamma_{S R}+\frac{\gamma_{R^{*} D}}{\gamma_{J^{*} D}+1}+1}\right)
$$

$$
I_{E}^{\mathrm{JRJS}}=\frac{1}{2} \log \left(1+\gamma_{E}^{\mathrm{JRJS}}\right)=\frac{1}{2} \log \left(1+\frac{\gamma_{S R} \frac{\gamma_{R E}}{\gamma_{J E}+1}}{\gamma_{S R}+\frac{\gamma_{R E}}{\gamma_{J E}+1}+1}\right)
$$

Remark 1: Generally, the optimal RN/JN selection scheme 332 should take into account the global SNR knowledge set 333 $\left\{\gamma_{S R}, \gamma_{R D}, \gamma_{R E}\right\}$. However, given the potentially excessive 334 implementational complexity overhead of the optimal selection 335 schemes and the unavailability of the global CSI, we employ 336 suboptimal selection schemes as in [12]. ${ }^{2}$ Furthermore, it is 337 commonly assumed that the average SNR of the eavesdropper 338 is available at the transmitter, which seems, somehow, not 339 reasonable. However, as stated in most of the literature, such as 340 [12]-[22], [24]-[28], and [30], provided that the eavesdropper 341 belongs to the network, which is also the case in our paper, 342 the related assumption might still be deemed reasonably. Addi- 343 tionally, as in [8], [11], [12], and [24], for mathematical conve- 344 nience, we assume that the relaying channels are independent 345 and identically distributed and that we have $\mathbb{E}\left[\gamma_{S R_{k}}\right]=\bar{\gamma}_{S R}, 346$ $\mathbb{E}\left[\gamma_{R_{k} D}\right]=\bar{\gamma}_{R D}$, and $\mathbb{E}\left[\gamma_{R_{k} E}\right]=\bar{\gamma}_{R E}$. The distances between 347 the relays are assumed to be much smaller than the distances 348 between relays and source/destination/eavesdropper; hence, the 349 corresponding path losses among the different relays are ap- 350 proximately the same. This assumption is reasonable both for 351 WSNs and for MANETs associated with a symmetric clustered 352 relay configuration, and it may be also satisfied as valid by 353 classic cellular systems in a statistical sense [11].

\section{Secure Transmission Without Jamming}

Here, we endeavor to characterize both the reliability and 356 security performance comprehensively of the TBRS scheme. 357 We first derive closed-form expressions for both the COP and 358 the SOP. Then, the RSR is introduced through the asymptotic 359 analysis of the COP and the SOP. Furthermore, we propose 360 the novel definition of the RSCP and the effective secrecy 361 throughput.

\footnotetext{
${ }^{2}$ To further alleviate the cooperation-related overhead, the selection criterion is based on the $R \rightarrow D$ link, since the second hop plays a dominant role in determining the received SNR, because the first hop corresponds to a multipleinput-single-output channel with the aid of multiple antennas, and hence, it is more likely to be better than the second hop. The optimal selection based on both hops is beyond the scope of this work.
} 


\section{A. COP and SOP}

364 When the perfect instantaneous CSI of the eavesdropper's 365 channel and even the legitimate users' channel is unavailable, 366 alternative definitions of the outage probability may be adopted 367 for the statistical characterization of the attainable secrecy 368 performance, particularly for delay-limited applications. Based 369 on [31, Def. 2], perfect secrecy cannot be achieved, when we 370 have $R_{e}<I_{E}$, where $I_{E}$ denotes the MI between the source 371 and the eavesdropper. Encountering this event is termed as a 372 secrecy outage. Furthermore, the destination is unable to flaw373 lessly decode the received codewords when $R_{0}>I_{D}$, which is 374 termed as a connection outage. The grade of reliability and the 375 grade of security maintained by a transmission scheme may be 376 then quantified by the COP and the SOP, respectively.

377 We continue by presenting our preliminary results versus the 378 point-to-point SNRs. Let us denote the cumulative distribution 379 function (CDF) and the probability density function (PDF) of a 380 random variable $X$ by $F_{X}(x)$ and $f_{X}(x)$, respectively. On one 381 hand, the PDF of $\gamma_{S R}$ using [29, eq. (15)] is given by

$$
\begin{array}{r}
f_{\gamma_{S R}}(x)=\sum_{n=0}^{N_{t}-1}\left(\begin{array}{c}
N_{t}-1 \\
n
\end{array}\right) \frac{\rho_{S R}^{2\left(N_{t}-1-n\right)}\left(\bar{\gamma}_{S R}\left(1-\rho_{S R}^{2}\right)\right)^{n}}{\bar{\gamma}_{S R}^{N_{t}}\left(N_{t}-1-n\right) !} \\
\times x^{N_{t}-1-n} e^{\frac{-x}{\gamma_{S R}}}
\end{array}
$$

382 whereas its $\mathrm{CDF}$ is given by

$$
\begin{aligned}
F_{\gamma_{S R}}(x)=1-\sum_{n=0}^{N_{t}-1} & \sum_{m=0}^{N_{t}-1-n}\left(\begin{array}{c}
N_{t}-1 \\
n
\end{array}\right) \\
& \times \frac{\rho_{S R}^{2\left(N_{t}-1-n\right)}\left(1-\rho_{S R}^{2}\right)^{n}}{m ! \bar{\gamma}_{S R}^{m}} x^{m} e^{\frac{-x}{\bar{\gamma} S R}} .
\end{aligned}
$$

383 On the other hand, for the instantaneous SNR of the $R \rightarrow$ $384 D$ hop, according to the principles of concomitants or induced 385 order statistics, the CDF of $\gamma_{R^{*} D}$ can be derived as in [32]

$$
F_{\gamma_{R^{*} D}}(y)=K_{r} \sum_{k=0}^{K_{r}-1}(-1)^{k}\left(\begin{array}{c}
K_{r}-1 \\
k
\end{array}\right) \frac{1-e^{\frac{-(k+1) y}{\left(k\left(1-\rho_{R D}^{2}\right)+1\right) \bar{\gamma}_{R D}}}}{k+1} .
$$

386 Thus, the COP of the TBRS strategy is given by

$$
P_{\text {co }}^{\mathrm{TBRS}}\left(R_{0}\right)=\operatorname{Pr}\left[I_{D}^{\mathrm{TBRS}}<R_{0}\right]=F_{\gamma_{D}^{\mathrm{TBRS}}}\left(\gamma_{t h}^{D}\right)
$$

387 where we have $\gamma_{t h}^{D}=2^{2 R_{0}}-1$, and the $\mathrm{CDF}$ of $\gamma_{D}^{\mathrm{TBRS}}$ can be 388 calculated as

$$
\begin{aligned}
& F_{\gamma_{D}^{\mathrm{TBRS}}}(x)=1 \\
& \quad-\int_{0}^{\infty}\left[1-F_{\gamma_{R^{*} D}}\left(\frac{x z+x(x+1)}{z}\right)\right] f_{\gamma_{S R^{*}}}(z+x) d z .
\end{aligned}
$$

389 Consequently, by substituting (8) and (10) into (12) and using 390 [33, eq. (3.471.9)], we arrive at a closed-form expression for

$$
F_{\gamma_{D}^{\mathrm{TBRS}}}(x) \text { as }
$$

$$
\begin{aligned}
F_{\gamma_{D}^{\mathrm{TBRS}}}(x)= & -2 \sum_{n=0}^{N_{t}-1} \sum_{k=0}^{K_{r}-1} \sum_{m=0}^{N_{t}-1-n}(-1)^{k} K_{r}\left(\begin{array}{c}
N_{t}-1 \\
n
\end{array}\right) \\
& \times\left(\begin{array}{c}
K_{r}-1 \\
k
\end{array}\right)\left(\begin{array}{c}
N_{t}-1-n \\
m
\end{array}\right) \\
& \times \frac{\rho_{S R}^{2\left(N_{t}-1-n\right)}\left(1-\rho_{S R}^{2}\right)^{n} x^{N_{t}-1-n-m}}{\left(N_{t}-1-n\right) !(k+1) \bar{\gamma}_{S R}^{N_{t}-n}} \\
& \times\left[\frac{\bar{\gamma}_{S R} x(x+1)}{\omega_{k} \bar{\gamma}_{R D}}\right]^{\frac{m+1}{2}} \\
& \times e^{-\left(\frac{\bar{\gamma}_{S R}+\omega_{k} \bar{\gamma}_{R D}}{\omega_{k} \bar{\gamma}_{S R} \bar{\gamma}_{R D}}\right) x} K_{m+1}\left(2 \sqrt{\frac{x(x+1)}{\omega_{k} \bar{\gamma}_{S R} \bar{\gamma}_{R D}}}\right)
\end{aligned}
$$

where we have $\omega_{k}=\left(k\left(1-\rho_{R D}^{2}\right)+1\right) /(k+1)$. Then, by 392 substituting $x=\gamma_{t h}^{D}$ into (13), we obtain $P_{\text {co }}^{\text {TBRS }}$.

Furthermore, the SOP of the TBRS strategy may be expressed as 394

$$
P_{\mathrm{so}}^{\mathrm{TBRS}}\left(R_{0}, R_{s}\right)=\operatorname{Pr}\left[I_{E}^{\mathrm{TBRS}}>R_{0}-R_{s}\right]=1-F_{\gamma_{E}^{\mathrm{TBRS}}}\left(\gamma_{t h}^{E}\right)
$$

where we have $\gamma_{t h}^{E}=2^{2\left(R_{0}-R_{s}\right)}-1$. Similarly, we may calcu- 395 late the $\mathrm{CDF}$ of $\gamma_{E}^{\mathrm{TBRS}}$ in (14) as

$$
\begin{aligned}
F_{\gamma_{E}^{\mathrm{TBRS}}}(x)= & 1-2 \sum_{n=0}^{N_{t}-1} \sum_{m=0}^{N_{t}-1-n}\left(\begin{array}{c}
N_{t}-1 \\
n
\end{array}\right)\left(\begin{array}{c}
N_{t}-1-n \\
m
\end{array}\right) \\
& \times \frac{\rho_{S R}^{2\left(N_{t}-1-n\right)}\left(1-\rho_{S R}^{2}\right)^{n} x^{N_{t}-1-n-m}}{\left(N_{t}-1-n\right) ! \bar{\gamma}_{S R}^{N_{t}-n}} \\
& \times\left[\frac{\bar{\gamma}_{S R} x(x+1)}{\bar{\gamma}_{R E}}\right]^{\frac{m+1}{2}} \\
& \times e^{-\left(\frac{\bar{\gamma}_{S R}+\bar{\gamma}_{R E}}{\bar{\gamma}_{S R} \bar{\gamma}_{R E}}\right) x} K_{m+1}\left(2 \sqrt{\frac{x(x+1)}{\bar{\gamma}_{S R} \bar{\gamma}_{R E}}}\right)
\end{aligned}
$$

Then, by substituting $x=\gamma_{t h}^{E}$ into (15), we can derive $P_{\text {so }}^{\text {TBRS }} .397$

The COP and the SOP in (11) and (14) characterize the at- 398 tainable reliability and security performance, respectively, and 399 can be regarded as the detailed requirements of accurate system 400 design. From the definition of COP and SOP, it is clear that 401 the reliability of the main link can be improved by increasing 402 the transmit SNR (or decreasing its data rate) to reduce the 403 COP, which unfortunately increases the risk of eavesdropping. 404 Thus, a tradeoff between reliability and security may be struck, 405 despite the fact that closed-from expressions cannot be obtained 406 as in [11]. Furthermore, we denote the minimal reliability and 407 security requirements by $v$ and $\delta$, where the feasible range of 408 the reliability constraint is $0<v<1$. Bearing in mind that 409 the COP is a monotonously increasing function of $R_{0}$, the 410 corresponding threshold of the codeword transmission rate is 411 $R_{0}^{\text {th }}=\arg \left\{P_{\mathrm{co}}^{\mathrm{TBRS}}\left(R_{0}\right)=v\right\}$, which leads to a lower bound of 412 the SOP, when we have $\left(R_{0}-R_{s}\right) \rightarrow R_{0}^{t h}$. Thus, the feasible 413 range of $\delta$ is $P_{\mathrm{so}}^{\mathrm{TBRS}}\left(R_{0}^{\text {th }}, 0\right)<\delta<1$. The preceding analysis 414 indicates that, given a reliability constraint $v$, the lower bound 415 of the security constraint is determined. 


\section{B. Reliability-Security Ratio}

418 Here, we will focus our attention on the asymptotic analysis 419 of the COP and the SOP in the high-SNR regime. Then, inspired 420 by [25], we introduce the concept of the RSR for characterizing 421 the direct relationship between reliability and security.

422 Proposition 1: Based on the asymptotic probabilities of $P_{\mathrm{co}}$ 423 and $P_{\text {so }}$ at high SNRs, ${ }^{3}$ the RSR is defined as

$$
P_{\mathrm{co}}\left(R_{0}\right)=\Lambda\left[1-P_{\mathrm{so}}\left(R_{0}, R_{s}\right)\right]
$$

424 where $\Lambda=\lim _{\eta \rightarrow \infty} P_{\mathrm{co}} /\left(1-P_{\mathrm{so}}\right)$, which represents the im425 provement in COP upon decreasing the SOP. More specifically, 426 since the reduction of the SOP/COP must be followed by an 427 improvement of COP/SOP, a lower $\Lambda$ implies that, when the 428 security is reduced, the reliability is improved, and vice versa. 429 Thus, for the TBRS scheme studied earlier, the RSR is derived 430 as (17), shown at the bottom of the page.

\section{Proof: The proof is given in Appendix B.}

432 Remark 2: It can be seen from the preceding expression 433 that the factor $\Lambda$ is independent of the transmit SNR, but 434 directly depends on the channel gains, the rate pair $\left(R_{0}, R_{s}\right)$, 435 and the number of TAs and relays. For a given $R_{s}$, reducing $436 R_{0}$ to enhance the reliability may erode the security, because $437\left(R_{0}-R_{s}\right)$ is also reduced. Conversely, increasing $R_{0}$ provides 438 more redundancy for protecting the security of the information, 439 but simultaneously, the reliability is reduced. Hence, the RSR 440 analysis underlines an important point of view concerning how 441 to balance the reliability versus security tradeoff by adjusting $442\left(R_{0}, R_{s}\right)$. Furthermore, as long as a CSI feedback delay exists, 443 the RSR has an intimate relationship with $\rho_{S R}$ and $\rho_{R D}$. It is 444 clear that the value of $\Lambda^{\text {TBRS }}$ decreases as $\rho_{R D}$ increases, which 445 is due to the fact that the relay selection process only improves 446 the reliability of the legitimate user. On the other hand, since 447 we always have the conclusion that $\sum_{k=0}^{K_{r}-1}(-1)^{k}\left(\begin{array}{c}K_{r}-1 \\ k\end{array}\right)\left(K_{r} /\right.$ $\left.448\left(k\left(1-\rho_{R D}^{2}\right)+1\right)\right)<1$, when $\sigma_{R D}^{2}$ and $\sigma_{R E}^{2}$ are comparable, $449 \Lambda^{\text {TBRS }}$ will be reduced as $\rho_{S R}$ increases. This observation 450 implies that, although both $P_{\text {co }}$ and $\left(1-P_{\text {so }}\right)$ are reduced 451 when the first-hop CSI becomes better, the improvement of

\footnotetext{
${ }^{3}$ Assume equal power allocation between $S$ and the relay, yielding $P_{S}=$ $P_{R}=P$, and define $\eta=P / N_{0}$ as the transmit SNR [24].
}

the reliability is more substantial than the security loss, as $\rho_{S R} 452$ increases.

\section{Effective Secrecy Throughput}

It should be noted that the COP and SOP metrics ignore the 455 correlation between these two outage events. More specifically, 456 in contrast to the point-to-point transmission case, since the 457 $S \rightarrow R$ link's SNR included in the MI expressions of (3) and 458 (4), the secrecy outage and the connection outage are definitely 459 not independent of each other. Therefore, it might be of limited 460 benefit in evaluating the reliability or the security separately. 461 We note furthermore that, although another metric referred to 462 as the secrecy throughput was introduced as the product of the 463 successful decoding probability and of the secrecy rate [21], 464 [22], this definition ignores the fact that a reliable transmission 465 may be insecure, and the SOP is not taken into consideration. 466 Hence, this metric is unable to holistically characterize the 467 efficiency of our scheme, while capable of achieving both re- 468 liable and secure transmission. Therefore, here, we redefine the 469 effective secrecy throughput as the probability of a successful 470 transmission (reliable and secure) multiplied by the secrecy 471 rate, namely, as $\varsigma=R_{s} P_{R \& S}$, where the RSCP is defined as 472

$$
P_{R \& S}=\operatorname{Pr}\left\{I_{D}>R_{0}, I_{E}<R_{0}-R_{s}\right\}
$$

Upon substituting the expressions of $I_{D}$ and $I_{E}$ in (3) and (4) 473 into (18), we can rewrite $P_{R \& S}$ for the TBRS strategy in (19), 474 shown at the bottom of the page.

Finally, using the corresponding CDFs and PDFs of (8)-(10) 476 from our previous analysis, we can obtain $P_{R \& S}^{\mathrm{TBRS}}$ in (20), 477 shown at the bottom of the next page, as well as the secrecy 478 throughput.

479

Furthermore, considering the asymptotic result for RSCP at 480 high SNRs in (20) by applying the approximation $K_{v}(x) \approx 481$ $(v-1) ! / 2(x / 2)^{v}$ and closing the highest terms of $\eta$ after 482 invoking the McLaurin series representation for the exponential 483 function, the asymptotic effective secrecy throughput can be 484 approximated as

485

Remark 3: Given the definition of COP, SOP, and the secrecy 486 throughput result of (21), shown at the bottom of the next page, 487 it can be shown that, for a fixed $R_{s}$, if $R_{0}$ is too small, although 488

$$
\Lambda^{\mathrm{TBRS}}=\frac{\left[\left(1-\rho_{S R}^{2}\right)^{N_{t}-1}+\sum_{k=0}^{K_{r}-1}(-1)^{k}\left(\begin{array}{c}
K_{r}-1 \\
k
\end{array}\right) \frac{K_{r} \sigma_{S R}^{2}}{\left[k\left(1-\rho_{R D}^{2}\right)+1\right] \sigma_{R D}^{2}}\right]\left(2^{2 R_{0}}-1\right)}{\left[N_{t}\left(1-\rho_{S R}^{2}\right)^{N_{t}-1}+\sigma_{S R}^{2} / \sigma_{R E}^{2}\right]\left(2^{2\left(R_{0}-R_{s}\right)}-1\right)}
$$

$$
\begin{aligned}
P_{R \& S}^{\mathrm{TBRS}} & =\operatorname{Pr}\left\{\left\{\gamma_{S R}>\gamma_{t h}^{D}, \gamma_{R^{*} D}>\frac{\gamma_{t h}^{D} \gamma_{S R}+\gamma_{S R}}{\gamma_{S R}-\gamma_{t h}^{D}}\right\} \cap\left[\left\{\gamma_{S R}>\gamma_{t h}^{E}, \gamma_{R^{*} E}<\frac{\gamma_{t h}^{E} \gamma_{S R}+\gamma_{S R}}{\gamma_{S R}-\gamma_{t h}^{E}}\right\} \cup\left\{\gamma_{S R}<\gamma_{t h}^{E}\right\}\right]\right\} \\
& =\operatorname{Pr}\left\{\gamma_{S R}>\gamma_{t h}^{D}, \gamma_{R^{*} D}>\gamma_{t h}^{D}+\frac{\gamma_{t h}^{D}\left(\gamma_{t h}^{D}+1\right)}{\gamma_{S R}-\gamma_{t h}^{D}}, \gamma_{R^{*} E}<\gamma_{t h}^{E}+\frac{\gamma_{t h}^{E}\left(\gamma_{t h}^{E}+1\right)}{\gamma_{S R}-\gamma_{t h}^{E}}\right\}
\end{aligned}
$$


$489 P_{R S}$ may be high (i.e., close to 1 ), the value of $\varsigma$ remains small. 490 By contrast, if $R_{0}$ is too large, the value of $P_{\text {co }}$ is close to 1 , 491 and therefore, $\varsigma$ will also become small. This observation is 492 also suitable for $R_{s}$. Thus, as pointed out in the RSR analysis, 493 it is elusive to improve both the reliability and the security 494 simultaneously, but both of them are equally crucial in terms 495 of the effective secrecy throughput, which depends on the rate 496 pair $\left(R_{0}, R_{s}\right)$.

497 Additionally, (21) also reveals that increasing the SNR would 498 drastically reduce the effective secrecy throughput. For high 499 transmit SNRs, a high reliability can indeed be perfectly guar500 anteed, but at the same time, the grade of the security is severely 501 degraded. However, the probability of a reliable and simultane502 ously secure transmission will tend toward zero. Hence, we may 503 conclude that there exists an optimal SNR, which achieves the 504 maximal secrecy throughput.

505 In conclusion, adopting the appropriate code rate pair and 506 transmit SNR is crucial for achieving the maximum effective 507 secrecy throughput, which can be formulated as

$$
\begin{aligned}
& \max _{R_{0}, R_{s}, \eta} \varsigma\left(R_{0}, R_{s}\right)=R_{s} P_{R \& S}^{\mathrm{TBRS}} \\
& \text { s.t. } P_{\text {co }} \leq v, P_{\text {so }} \leq \delta, 0<R_{s}<R_{0}
\end{aligned}
$$

508 where $v$ and $\delta$ denote the system's reliability and security 509 requirements. Unfortunately, it is quite a challenge to find 510 the closed-form optimal solution to this problem due to the 511 complexity of the expressions. Although suboptimal solutions 512 can be found numerically (with the aid of gradient-based search 513 techniques), the secrecy throughput optimization problem and 514 the corresponding complexity analysis and performance com515 parisons are beyond the scope of this work.

\section{6}

\section{SECURE Transmission With JAMming}

517 Here, we consider the extension of the aforementioned relay 518 selection approaches to systems additionally invoking relay- aided jamming. JRJS is based on the outdated but perfectly 519 estimated CSI, and the details have been presented in Section II. 520 We would also like to investigate the security performance 521 from an outage-based perspective. The COP, SOP, RSCP, and 522 effective secrecy throughput will be included.

\section{A. COP and SOP}

It is plausible that the main differences between the JRJS and 525 TBRS schemes are determined by the instantaneous SNR of the 526 $R \rightarrow D$ hop, where, now, a jammer is included. Based on our 527 preliminary results detailed for the point-to-point SNRs in (8) 528 and (10), we now focus our attention on the statistical analysis 529 of the SNR, including $J^{*}$. As stated for the JRJS scheme in 530 Section II, $J^{*}$ corresponds to the lowest $\tilde{\gamma}_{R_{k} D}$ and is selected 531 from the set $\left\{\mathcal{R}-R^{*}\right\}$. Recalling that $R^{*}$ is the best relay 532 of the second hop, we have $\tilde{\gamma}_{J^{*} D}=\min _{R_{k} \in \mathcal{R}-R^{*}}\left\{\tilde{\gamma}_{R_{k} D}\right\} \triangleq 533$ $\min _{R_{k} \in \mathcal{R}}\left\{\tilde{\gamma}_{R_{k} D}\right\}$ for $K_{r}>1$. Using the induced order statis- 534 tics, the corresponding CDF of $\gamma_{R^{*} D}$ is presented in (10), 535 whereas the PDF of $\gamma_{J^{*} D}$ can be formulated as

$$
f_{\gamma_{J^{*} D}}(x)=\frac{K_{r} \exp \left(\frac{-K_{r} x}{\left[\left(K_{r}-1\right)\left(1-\rho_{R D}^{2}\right)+1\right] \bar{\gamma}_{J D}}\right)}{\left[\left(K_{r}-1\right)\left(1-\rho_{R D}^{2}\right)+1\right] \bar{\gamma}_{J D}} .
$$

Although the relay and jammer selection processes are not 537 entirely disjoint, we may exploit the assumption that $\gamma_{R^{*} D}$ and 538 $\gamma_{J * D}$ are independent of each other, which is valid when the 539 number of relays is sufficiently high, as justified in [24]. Let us 540 define the signal-to-interference-plus-noise ratio of the second 541 hop as $\xi_{D}=\gamma_{R^{*} D} /\left(\gamma_{J^{*} D}+1\right)$, using (10) and (23), whose 542 CDF can be formulated as

$$
F_{\xi_{D}}(x)=1-K_{r} \sum_{k=0}^{K_{r}-1}(-1)^{k}\left(\begin{array}{c}
K_{r}-1 \\
k
\end{array}\right) \frac{\varphi_{k} e^{\frac{-x}{\gamma_{R D} \omega_{k}}}}{(k+1)\left(x+\varphi_{k}\right)}
$$

where we have $\varphi_{k}=\lambda K_{r} \omega_{k} /\left(\left[\left(K_{r}-1\right)\left(1-\rho_{R D}^{2}\right)+1\right](1-\lambda)\right) . \quad 544$

$$
\begin{aligned}
P_{R \& S}^{\mathrm{TBRS}}= & \int_{\gamma_{t h}^{D}}^{\infty}\left[1-F_{\gamma_{R^{*}}}\left(\gamma_{t h}^{D}+\frac{\gamma_{t h}^{D}\left(\gamma_{t h}^{D}+1\right)}{x-\gamma_{t h}^{D}}\right)\right] F_{\gamma_{R^{*}}}\left(\gamma_{t h}^{E}+\frac{\gamma_{t h}^{E}\left(\gamma_{t h}^{E}+1\right)}{x-\gamma_{t h}^{E}}\right) f_{\gamma_{S R^{*}}}(x) d x \\
\approx & 2 \sum_{n=0}^{N_{t}-1} \sum_{k=0}^{K_{r}-1} \sum_{m=0}^{N_{t}-1-n}(-1)^{k}\left(\begin{array}{c}
K_{r}-1 \\
k
\end{array}\right)\left(\begin{array}{c}
N_{t}-1 \\
n
\end{array}\right)\left(\begin{array}{c}
N_{t}-1-n \\
m
\end{array}\right) \frac{K_{r} \rho_{S R}^{2\left(N_{t}-1-n\right)}\left(1-\rho_{S R}^{2}\right)^{n}\left(\gamma_{t h}^{D}\right)^{N_{t}-1-n-m}}{\left(N_{t}-1-n\right) !(k+1) \bar{\gamma}_{S R}^{N_{t}-n-(m+1) / 2}} \\
& \times \exp \left[-\left(\frac{\gamma_{t h}^{D}}{\bar{\gamma}_{S R}}+\frac{\gamma_{t h}^{D}}{\omega_{k} \bar{\gamma}_{R D}}\right)\right]\left[\left(\frac{\gamma_{t h}^{D}\left(\gamma_{t h}^{D}+1\right)}{\omega_{k} \bar{\gamma}_{R D}}\right)^{\frac{m+1}{2}} K_{m+1}\left(2 \sqrt{\frac{\gamma_{t h}^{D}\left(\gamma_{t h}^{D}+1\right)}{\omega_{k} \bar{\gamma}_{S R} \bar{\gamma}_{R D}}}\right)\right. \\
& \left.-\exp \left(\frac{-\gamma_{t h}^{E}}{\bar{\gamma}_{R E}}\right)\left(\frac{\gamma_{t h}^{D}\left(\gamma_{t h}^{D}+1\right)}{\omega_{k} \bar{\gamma}_{R D}}+\frac{\gamma_{t h}^{E}\left(\gamma_{t h}^{E}+1\right)}{\bar{\gamma}_{R E}+\gamma_{t h}^{D}-\gamma_{t h}^{E}}\right)^{\frac{m+1}{2}} K_{m+1}\left(2 \sqrt{\frac{\gamma_{t h}^{D}\left(\gamma_{t h}^{D}+1\right)}{\omega_{k} \bar{\gamma}_{S R} \bar{\gamma}_{R D}}+\frac{\gamma_{t h}^{E}\left(\gamma_{t h}^{E}+1\right)}{\bar{\gamma}_{S R}\left(\bar{\gamma}_{R E}+\gamma_{t h}^{D}-\gamma_{t h}^{E}\right)}}\right)\right]
\end{aligned}
$$

$$
\tilde{\varsigma}^{\operatorname{TBRS}}\left(R_{0}, R_{s}, \eta\right)=R_{s}\left\{1-\left[\frac{N_{t}\left(1-\rho_{S R}^{2}\right)^{N_{t}-1}}{\sigma_{S R}^{2}}+\sum_{k=0}^{K_{r}-1} \frac{K_{r}(-1)^{k}}{\left[k\left(1-\rho_{R D}^{2}\right)+1\right] \sigma_{R D}^{2}}\left(\begin{array}{c}
K_{r}-1 \\
k
\end{array}\right)\right] \times \frac{2^{2 R_{0}}-1}{\eta}\right\} \frac{2^{2\left(R_{0}-R_{s}\right)}-1}{\sigma_{R E}^{2} \eta}
$$


545 As far as the eavesdropper is concerned, $\gamma_{R^{*} E}$ and $\gamma_{J^{*} E}$ 546 are independent and exponentially distributed. Furthermore, for $547 \xi_{E}=\gamma_{R^{*} E} /\left(\gamma_{J^{*} E}+1\right)$, we have

$$
F_{\xi_{E}}(x)=1-\frac{\phi}{x+\phi} e^{\frac{-x}{\bar{\gamma}_{R E}}}
$$

548 where $\phi=\lambda /(1-\lambda)$. According to the definition of COP and 549 SOP in Section III-A, we can obtain the following closed-form 550 approximations of the COP and the SOP. ${ }^{4}$

551 Lemma 1: The COP and the SOP of the JRJS strategy 552 associated with feedback delays are approximated by

$$
\begin{aligned}
P_{\mathrm{co}}^{\mathrm{JRJS}}\left(R_{0}\right) \approx & 1-\sum_{n=0}^{N_{t}-1} \sum_{k=0}^{K_{r}-1} \sum_{m=0}^{N_{t}-1-n}\left(\begin{array}{c}
N_{t}-1 \\
n
\end{array}\right) \\
& \times\left(\begin{array}{c}
K_{r}-1 \\
k
\end{array}\right)\left(\begin{array}{c}
N_{t}-1-n \\
m
\end{array}\right) \\
& \times \frac{(-1)^{k}\left(K_{r}+1\right) \rho_{S R}^{2\left(N_{t}-1-n\right)}\left(1-\rho_{S R}^{2}\right)^{n}}{\left(N_{t}-1-n\right) !(k+1) \bar{\gamma}_{S R}^{N_{t}-n}} \\
& \times \frac{\Gamma(m+2) \hat{\varphi}_{k}\left(\gamma_{t h}^{D}\right)^{N_{t}-n}\left(\gamma_{t h}^{D}+1\right)^{m+1}}{\left(\gamma_{t h}^{D}+\hat{\varphi}_{k}\right)^{m+2}} \\
& \times \exp \left[-\frac{\gamma_{t h}^{D}\left(\hat{\varphi}_{k}-1\right)}{\bar{\gamma}_{S R}\left(\gamma_{t h}^{D}+\hat{\varphi}_{k}\right)}\right] \\
& \times \Gamma\left(-m-1, \frac{\gamma_{t h}^{D}\left(\gamma_{t h}^{D}+1\right)}{\bar{\gamma}_{S R}\left(\gamma_{t h}^{D}+\hat{\varphi}_{k}\right)}\right)
\end{aligned}
$$

553 where $\quad \hat{\varphi}_{k}=K_{r} \lambda \omega_{k} \eta \sigma_{R D}^{2} /\left(\left[\left(K_{r}-1\right)\left(1-\rho_{R D}^{2}\right)+1\right](1-\right.$ $\left.554 \lambda) \eta \sigma_{R D}^{2}+K_{r}\right)$, and

$$
\begin{aligned}
& P_{\mathrm{so}}^{\mathrm{JRJS}}\left(R_{0}, R_{s}\right) \approx \sum_{n=0}^{N_{t}-1} \sum_{m=0}^{N_{t}-1-n}\left(\begin{array}{c}
N_{t}-1 \\
n
\end{array}\right) \\
& \times \frac{\rho_{S R}^{2\left(N_{t}-1-n\right)}\left(1-\rho_{S R}^{2}\right)^{n}}{m ! \bar{\gamma}_{S R}^{m}} \\
& \times \frac{\left(2 \gamma_{t h}^{E}\right)^{m} \phi}{\left(2 \gamma_{t h}^{E}+\phi\right)} \exp \left[-\left(\frac{2 \gamma_{t h}^{E}}{\bar{\gamma}_{S R}}+\frac{2 \gamma_{t h}^{E}}{\bar{\gamma}_{R E}}\right)\right] .
\end{aligned}
$$

555 Proof: The proof is given in Appendix B.

556 The feasible range of the reliability constraint is similar to 557 that of the TBRS strategy, and hence, it is omitted here.

\section{B. Reliability-Security Ratio}

559 Lemma 2: Recalling the definition in Section III, the RSR 560 for the JRJS strategy may be expressed in (28), shown at the 561 bottom of the page.

${ }^{4}$ When we have $\lambda \rightarrow 1$, (24) will degenerate into the TBRS case seen in (10). The performance analysis of the JRJS will be presented separately in the following, since several approximations have to be included.
It can be seen from the previous expression that, in contrast 562 to the analysis of the TBRS strategy operating without jam- 563 ming, for a fixed SNR threshold, the CDF of the second-hop 564 SNR will converge to a nonzero limit. We also find that this 565 limit is determined by the power sharing ratio between the 566 relay and the jammer. Furthermore, according to the analy- 567 sis of the TBRS strategy, for $\eta \rightarrow \infty$, we have $F_{\gamma_{S R^{*}}}(x) \rightarrow 568$ 0 . Thus, by exploiting the tight upper bound that $\gamma_{D}^{\text {TBRS }} \leq 569$ $\min \left\{\gamma_{S R}, \gamma_{R^{*} D}\right\}$ and $\gamma_{E}^{\mathrm{TBRS}} \leq \min \left\{\gamma_{S R}, \gamma_{R^{*} E}\right\}$, we have 570 $P_{\text {co }}^{\mathrm{JRJS}, \infty} \rightarrow F_{\gamma_{\xi_{D}}}\left(\gamma_{t h}^{D}\right)$ and $1-\bar{P}_{\mathrm{so}}^{\mathrm{JRJS}, \infty} \rightarrow F_{\gamma_{\xi_{E}}}\left(\gamma_{t h}^{E}\right)$. Finally, 571 substituting the corresponding results into (16), we arrive at the 572 RSR of the JRJS strategy.

573

Remark 4: It can be seen from the RSR expression of (28) 574 again that the rate-pair setting $\left(R_{0}, R_{s}\right)$ has an inconsistent 575 influence on the RSR, and hence, we have to carefully adjust $R_{0} 576$ and $R_{s}$ to balance the reliability versus security performance. 577 Let us now focus our attention on the differences between the 578 JRJS scheme and the TBRS arrangement.

579

First, we may find that the power sharing ratio $\lambda$ between 580 the relay and the jammer plays a very important role. The 581 optimization of $\lambda$ will be investigated from an effective secrecy 582 throughput optimization point of view in the following. 583

Second, it is plausible that, in contrast to the behavior of the 584 TBRS strategy, $\Lambda^{\mathrm{JRJS}}$ of (28) is only related to the delay of the 585 second hop, but it is still a monotonically decreasing function of 586 $\rho_{R D}$. This implies that the improvement of the channel quality 587 of the JRJS will achieve a more pronounced COP improvement 588 than the associated SOP improvement. Furthermore, recalling 589 that the RSR is considered in the high-SNR region, it has no 590 dependence on the first hop quality. This is due to the fact that 591 if the first-hop channel quality is sufficiently high for ensuring 592 a successful transmission, the asymptotic CDFs of $\xi_{D}$ and $\xi_{E} 593$ in (29) and (30) associated with $\eta \rightarrow \infty$ will converge to a 594 nonzero limit at high SNRs, which ultimately dominates the 595 COP and the SOP.

\section{Effective Secrecy Throughput}

Before proceeding to the effective secrecy throughput analy- 598 sis, we also have to investigate the RSCP.

599

Lemma 3: The RSCP of our JRJS strategy may be approxi- 600 mated as in (31), shown at the bottom of the next page, where 601 we have $\theta_{1, k}=\left(\gamma_{t h}^{D}\left(\gamma_{t h}^{D}+1\right)\right) /\left(\gamma_{t h}^{D}+\hat{\varphi}_{k}\right), \theta_{2}=\gamma_{t h}^{D}-\gamma_{t h}^{E}+602$ $\left(\gamma_{t h}^{E}\left(\gamma_{t h}^{E}+1\right) /\left(\gamma_{t h}^{E}+\hat{\phi}\right)\right)$, and $\hat{\phi}=\lambda \eta \sigma_{R E}^{2} /\left((1-\lambda) \eta \sigma_{R E}^{2}+1\right) .603$

Proof: The proof is given in Appendix C.

604

Apart from the rate pair $\left(R_{0}, R_{s}\right)$, the aforementioned $P_{R \& S}^{\text {JRJS }} 605$ of (31) is also a function of the power sharing ratio $\lambda$ between 606 the selected relay and the jammer.

607

Given the complexity of the RSCP expression, it is quite 608 a challenge to find a closed-form result for maximizing the 609

$$
\Lambda^{\mathrm{JRJS}}=\frac{\left(2^{2 R_{0}}-1\right)}{\left(2^{2\left(R_{0}-R_{s}\right)}-1\right)} \sum_{k=0}^{K_{r}-1}\left(\begin{array}{c}
K_{r}-1 \\
k
\end{array}\right) \frac{(-1)^{k} K_{r}\left[\left(K_{r}-1\right)\left(1-\rho_{R D}^{2}\right)+1\right]\left[\left(\lambda^{-1}-1\right)\left(2^{2\left(R_{0}-R_{s}\right)}-1\right)+1\right]}{\left[\left(K_{r}-1\right)\left(1-\rho_{R D}^{2}\right)+1\right](k+1)\left(\lambda^{-1}-1\right)\left(2^{2 R_{0}}-1\right)+K_{r}\left[k\left(1-\rho_{R D}^{2}\right)+1\right]}
$$


610 effective secrecy throughput that $\max _{0<\lambda<1} \varsigma=R_{s} P_{R \& S}^{\mathrm{JRJS}}$. Al611 ternatively, we can focus on the asymptotic analysis in the high612 SNR region and try to find a general closed-form solution for $\lambda$. 613 Specifically, when we have $\eta \rightarrow \infty, P_{R \& S}^{J R J S}$ will be dominated 614 by the channel quality of the second hop; hence, we have

$$
\begin{aligned}
P_{R \& S}^{\mathrm{JRJS}, \infty}\left(R_{0}, R_{s}, \lambda\right) & \approx \operatorname{Pr}\left\{\xi_{D}>\gamma_{t h}^{D}, \xi_{E}<\gamma_{t h}^{E}\right\} \\
& =\left[1-F_{\xi_{D}}\left(\gamma_{t h}^{D}\right)\right] F_{\xi_{E}}\left(\gamma_{t h}^{E}\right)
\end{aligned}
$$

615 where the approximation is based on the fact that, in contrast to 616 both $F_{\xi_{D}}\left(\gamma_{t h}^{D}\right)$ and $F_{\xi_{E}}\left(\gamma_{t h}^{E}\right)$, which converge to a nonzero limit 617 regardless of $\eta$, the first hop's $F_{\gamma_{S R}}(x)$ will tend to zero, and 618 hence, it can be neglected. Substituting the asymptotic results 619 of (29) and (30) into (33), we can obtain $P_{R \& S}^{\mathrm{JRJS}, \infty}$. In contrast to 620 the TBRS case operating without jamming, as the SNR tends to $621 \infty$, the RSCP will tend to a nonzero value and, upon increasing 622 the transmit SNR beyond a certain limit, will no longer increase 623 the effective secrecy throughput.

624 Then, based on (32), we arrive at the approximated optimal 625 value $\lambda_{\text {opt }}$, which is the solution of the following equation:

$$
\frac{\partial P_{R \& S}^{\mathrm{JRJS}, \infty}\left(R_{0}, R_{s}, \lambda\right)}{\partial \lambda}=0 .
$$

626 Then, by exploiting the approximation of $\left[k\left(1-\rho_{R D}^{2}\right)+1\right] /$ $627(k+1) \approx 1-\rho_{R D}^{2}$ in (29) for a large $\rho_{R D}$ (practically, the CSI 628 delay is small, and $\rho_{R D} \rightarrow 1$ ), we have

$$
\lambda_{\text {subopt }}=\frac{\sqrt{\left[\left(K_{r}-1\right)\left(1-\rho_{R D}^{2}\right)+1\right] \gamma_{t h}}}{\sqrt{\left[\left(K_{r}-1\right)\left(1-\rho_{R D}^{2}\right)+1\right] \gamma_{t h}}+\sqrt{K_{r}\left(1-\rho_{R D}^{2}\right)}}
$$

629 where $\gamma_{t h}=\left(2^{2 R_{0}}-1\right)\left(2^{2\left(R_{0}-R_{s}\right)}-1\right)$. It is clear that this 630 value is determined by the number of relays and $\left(R_{0}, R_{s}\right)$.

\section{Numerical Results}

632 Both our numerical and Monte Carlo simulation results are 633 presented here for verifying the theoretical PLS performance 634 analysis of the multiple-relay-aided network under CSI feed-

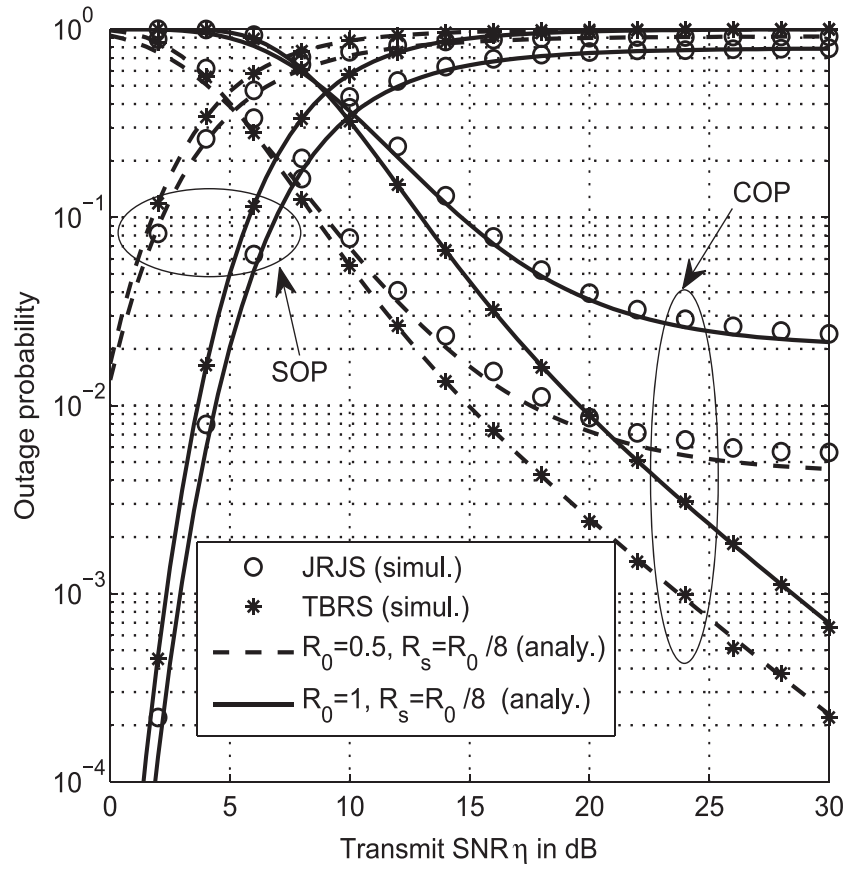

Fig. 2. COP and SOP versus transmit SNR for the TBRS and JRJS strategies in conjunction with different rate pairs, for $N_{t}=K_{r}=3, f_{d} T_{d}=0.1$, and $\lambda=1 / 10$.

back delays. Explicitly, the COP, SOP, RSCP, and RSR are 635 validated for both the TBRS and JRJS strategies. Furthermore, 636 the effects of feedback delays and system parameters (including 637 the transmission rate pair $\left(R_{0}, R_{s}\right)$ and the power sharing ratio 638 $\lambda$ between the relay and the jammer) on the achievable effective 639 secrecy throughput are evaluated. The Rayleigh fading model 640 is employed for characterizing all communication links in our 641 system. Additionally, we set the total power to $P=1$ and 642 $\sigma_{S R}^{2}=\sigma_{R D}^{2}=\sigma_{R E}^{2}=1$, and used $T_{d_{S R}}=T_{d_{R D}}=T_{d}$. 643

Fig. 2 plots the COP and the SOP versus the transmit SNR for 644 both the TBRS and JRJS strategies in conjunction with different 645 rate pairs. The analytical lines are plotted by using (11) and (14) 646 for the TBRS strategy and by using (26) and (27) for the JRJS 647

$$
\begin{aligned}
& P_{R \& S}^{\mathrm{JRJS}}\left(R_{0}, R_{s}, \lambda\right) \approx \sum_{n=0}^{N_{t}-1} \sum_{k=0}^{K_{r}-1} \sum_{m=0}^{N_{t}-1-n}(-1)^{k}\left(\begin{array}{c}
N_{t}-1 \\
n
\end{array}\right)\left(\begin{array}{c}
K_{r}-1 \\
k
\end{array}\right)\left(\begin{array}{c}
N_{t}-1-n \\
m
\end{array}\right) \\
& \times \frac{K_{r} \rho_{S R}^{2\left(N_{t}-1-n\right)}\left(1-\rho_{S R}^{2}\right)^{n} \hat{\varphi}_{k}\left(\gamma_{t h}^{D}\right)^{N_{t}-1-n-m}}{\left(N_{t}-1-n\right) !(k+1) \bar{\gamma}_{S R}^{N_{t}-n}\left(\gamma_{t h}^{D}+\hat{\varphi}_{k}\right) e^{\frac{\gamma_{t h}^{D}}{\bar{\gamma}_{S R}}+\frac{\gamma_{t h}^{D}}{\bar{\gamma}_{R D} \omega_{k}}}} \\
& \times\left\{\theta_{1, k}^{m+1} e^{\frac{\theta_{1}}{\bar{\gamma}_{S R}}} \Gamma(m+2) \Gamma\left(-m-1, \frac{\theta_{1, k}}{\bar{\gamma}_{S R}}\right)-\frac{\hat{\phi} e^{-\gamma_{t h}^{E} / \bar{\gamma}_{R E}}}{\left(\gamma_{t h}^{E}+\phi\right)\left(\theta_{1, k}-\theta_{2}\right)} \Gamma(m+3)\right. \\
& \times\left[\theta_{2}^{m+2} e^{\frac{\theta_{2}}{\bar{\gamma}_{S R}}} \Gamma\left(-m-2, \frac{\theta_{2}}{\bar{\gamma}_{S R}}\right)-\theta_{1, k}^{m+2} e^{\frac{\theta_{1}}{\bar{\gamma}_{S R}}} \Gamma\left(-m-2, \frac{\theta_{1, k}}{\bar{\gamma}_{S R}}\right)\right]+\Gamma(m+2)\left(\gamma_{t h}^{D}-\gamma_{t h}^{E}\right) \\
& \left.\times\left[\theta_{2}^{m+1} e^{\frac{\theta_{2}}{\bar{\gamma}_{S R}}} \Gamma\left(-m-1, \frac{\theta_{2}}{\bar{\gamma}_{S R}}\right)-\theta_{1, k}^{m+1} e^{\frac{\theta_{1}}{\bar{\gamma}_{S R}}} \Gamma\left(-m-1, \frac{\theta_{1, k}}{\bar{\gamma}_{S R}}\right)\right]\right\}
\end{aligned}
$$




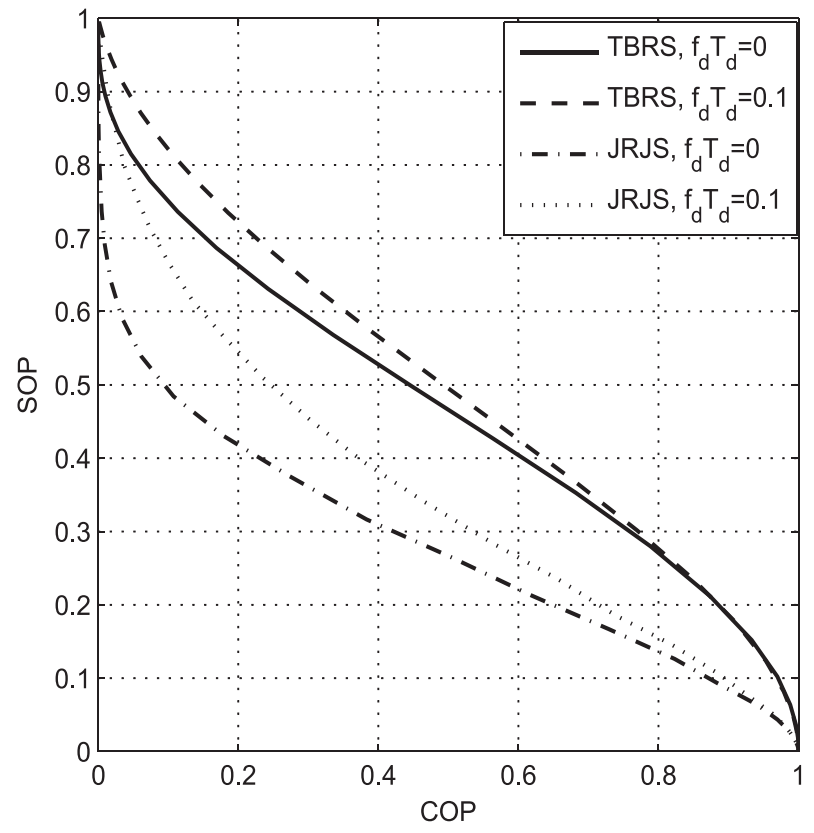

Fig. 3. SOP versus COP for the TBRS and JRJS strategies with different feedback delays for $N_{t}=K_{r}=3, R_{s}=R_{0} / 8$, and $\lambda=1 / 10$.

648 case, respectively. It can be clearly seen from the figure that the 649 analytical and simulated outage probability curves match well, 650 which confirms the accuracy of the mathematical analysis. As 651 expected, compared with the TBRS strategy, the SOP of the 652 JRJS strategy is much better, whereas the COP is worse. We 653 can also find that both the COP and the SOP will converge to an 654 outage floor at high SNRs for the JRJS strategy. The reason for 655 this is that the jammer also imposes interference on the destina656 tion and the interference inflicted increases with the SNR. Thus, 657 the designers have to take into account the tradeoff between 658 the reliability and the security and the interference imposed on $659 D$, particularly when considering the JRJS strategy. Moreover, 660 we can observe in Fig. 2 that increasing the transmission rate 661 decreases the COP and increases the SOP.

662 Fig. 3 further characterizes the SOP versus COP for both the 663 TBRS and JRJS strategies based on the numerical results in 664 Fig. 2, which shows the tradeoff between the reliability and the 665 security. It can be seen from the figure that the SOP decreases as 666 the COP increases, and for a specific COP, the SOP of the JRJS 667 scheme is strictly lower than that of TBRS. This confirms that 668 the JRJS scheme performs better than the conventional TBRS 669 scheme. Furthermore, the CSI feedback delay will also degrade 670 the system tradeoff performance.

671 Fig. 4 illustrates the RSCP versus transmit SNR for the 672 TBRS strategy in the context of different network configura673 tions, including different rate pairs, different number of relays, 674 and both perfect and outdated CSI feedback scenarios. The 675 analytical lines are plotted by using the approximation in (20). $676 \mathrm{We}$ may conclude from the figure that the rate-pair setting $677\left(R_{0}, R_{s}\right)$ determines both the reliability and security transmis678 sion performance. These curves also show that the RSCP is a 679 concave function of the transmit SNR, whereas the continued 680 boosting of the SNR would only decrease the probability of 681 a successful transmission. We can observe from Fig. 4 that,

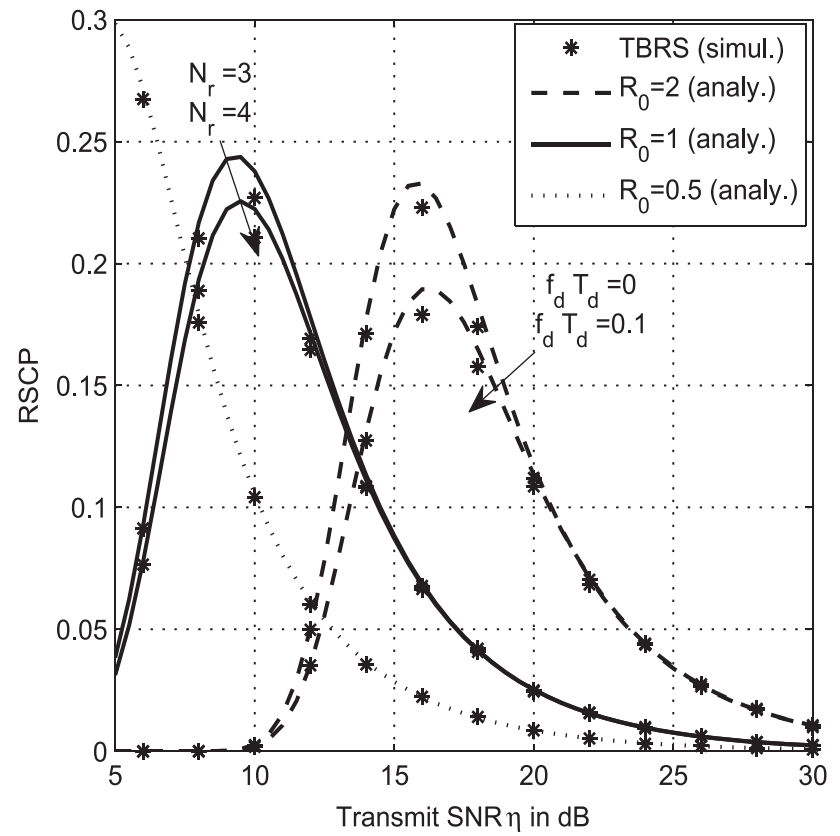

Fig. 4. RSCP versus transmit SNR for the TBRS strategy with different rate pairs for $N_{t}=K_{r}=3, f_{d} T_{d}=0.1$.

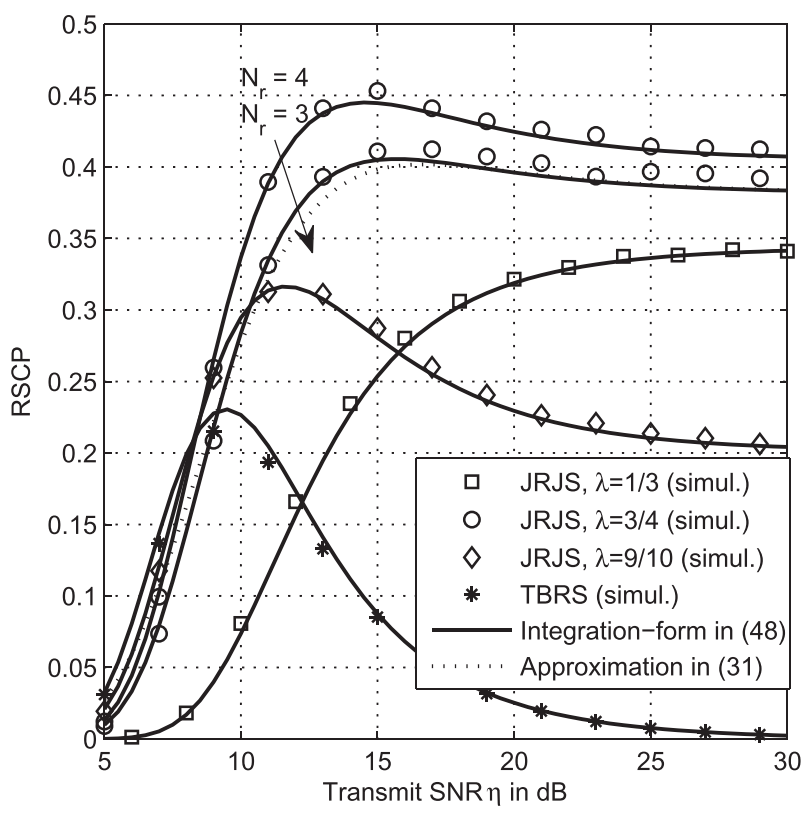

Fig. 5. RSCP versus transmit SNR for the JRJS strategy for different power sharing ratios $\lambda$ and for $N_{t}=K_{r}=3, f_{d} T_{d}=0.1$, and $R_{0}=1$, $R_{s}=R_{0} / 8$.

for a high transmit SNR, total reliability can be guaranteed, 682 whereas the associated grade of security is severely eroded. 683 Furthermore, increasing the number of relays and decreasing 684 the feedback delay will improve both the reliability and security 685 performance.

686

The RSCP of the JRJS strategy is presented in Fig. 5 for 687 different power sharing ratios between relaying and jamming. 688 Both the integration form (45) and the approximated closed 689 form in (31) match well with the Monte Carlo simulations. 690 The performance of the TBRS strategy is also included for 691 comparison. The JRJS scheme outperforms the TBRS operating 692 

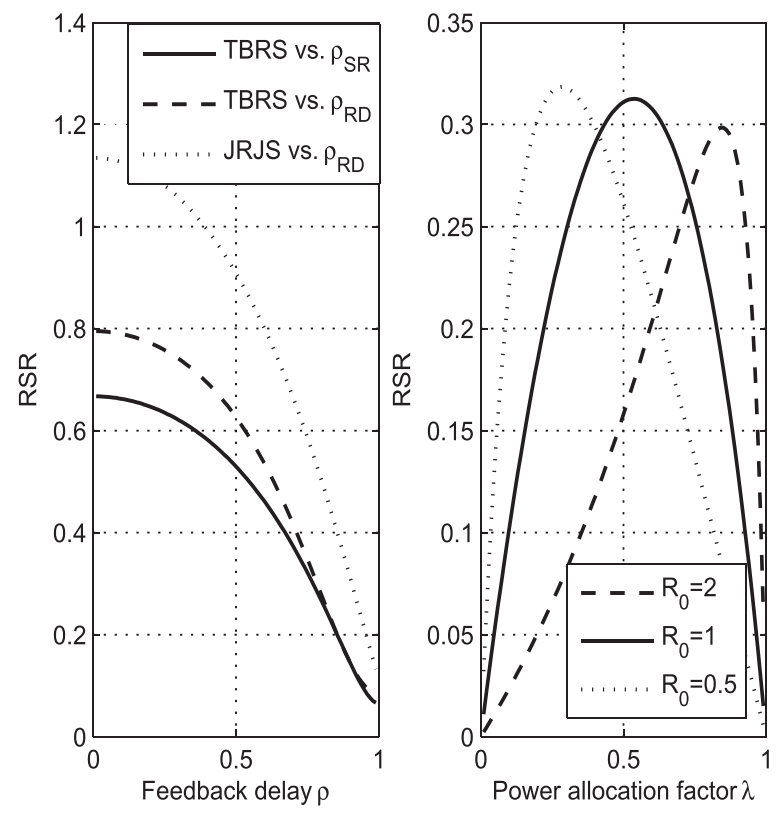

Fig. 6. RSR versus feedback delay coefficient $\left(R_{0}=1, R_{s}=R_{0} / 8, \lambda=\right.$ $3 / 4)$ and power sharing ratio $\lambda\left(R_{s}=R_{0} / 8, \rho_{S R}=\rho_{R D}=0.9\right)$ for the TBRS and JRJS strategies, with $N_{t}=K_{r}=3$.

693 without jamming under the scenario considered when encoun694 tering comparable relay-destination and relay-eavesdropper 695 channels. For some extreme configurations (when the relay696 eavesdropper links are comparatively weak), this statement 697 may not hold, but this scenario is beyond the scope of this 698 paper. The maximum RSCP appears at about $\eta=15 \mathrm{~dB}$ 699 for the JRJS strategy using $\lambda=3 / 4$, whereas it is $\eta=10 \mathrm{~dB}$ 700 for the TBRS strategy. Furthermore, as expected, increasing the 701 number of available relays and jamming nodes will always be 702 able to improve the reliability and security performance. How703 ever, the continued boosting of the jammer's power (decreasing $704 \lambda$ ) will not always improve the overall performance, because 705 the interference improves initially the security, but then, it starts 706 to reduce the reliability as $\lambda$ decreases. This further motivates 707 the designer to carefully take into account the power sharing 708 between relaying and jamming. The effect of the rate-pair 709 setting on the security and reliability of the JRJS strategy is 710 neglected here, which follows a similar trend to that of the 711 TBRS strategy.

712 Fig. 6 characterizes the RSR versus feedback delay and 713 power sharing ratio for both TBRS and JRJS, in which the 714 RSR curves are plotted by using (17) and (28), respectively. 715 The first illustration shows that the RSR decreases as the delay 716 coefficients $\left(\rho_{S R}\right.$ and $\left.\rho_{R D}\right)$, which confirms that the im717 provement of reliability becomes more pronounced than the 718 reduction of the security as the feedback delay decreases. 719 This observation implies an improvement in terms of the 720 security-reliability tradeoff. In addition, the RSR versus $\rho_{R D}$ 721 is larger than that of $\rho_{S R}$, which indicates that the impact of the 722 second-hop CSI feedback delay is more prominent. The other 723 illustration in the right demonstrates that the RSR is a concave 724 function of the power sharing ratio, which reflects the tradeoff 725 between the reliability and the security struck by adjusting $\lambda$.

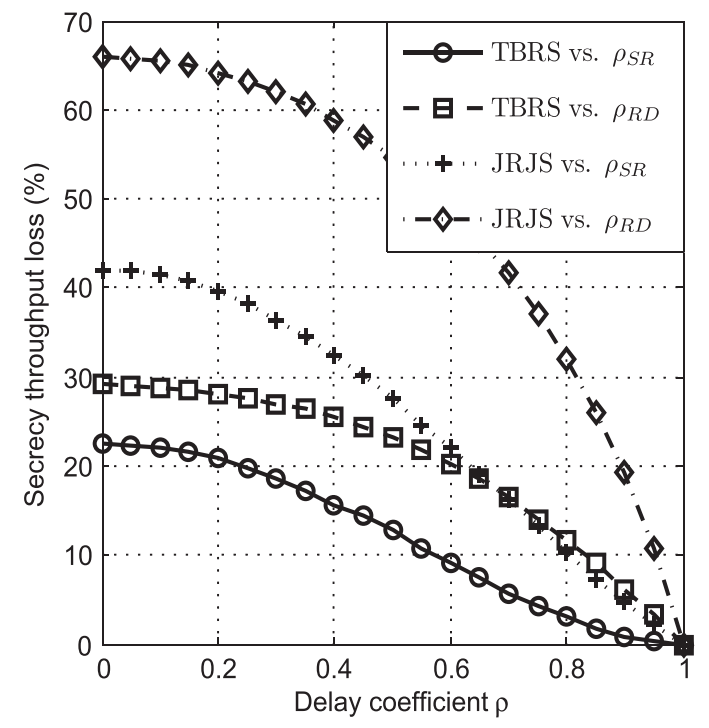

Fig. 7. Percentage secrecy throughput loss versus delay coefficients with $N_{t}=K_{r}=3, R_{0}=1, R_{s}=R_{0} / 8, \lambda=3 / 4$, and $\eta=10 \mathrm{~dB}$.

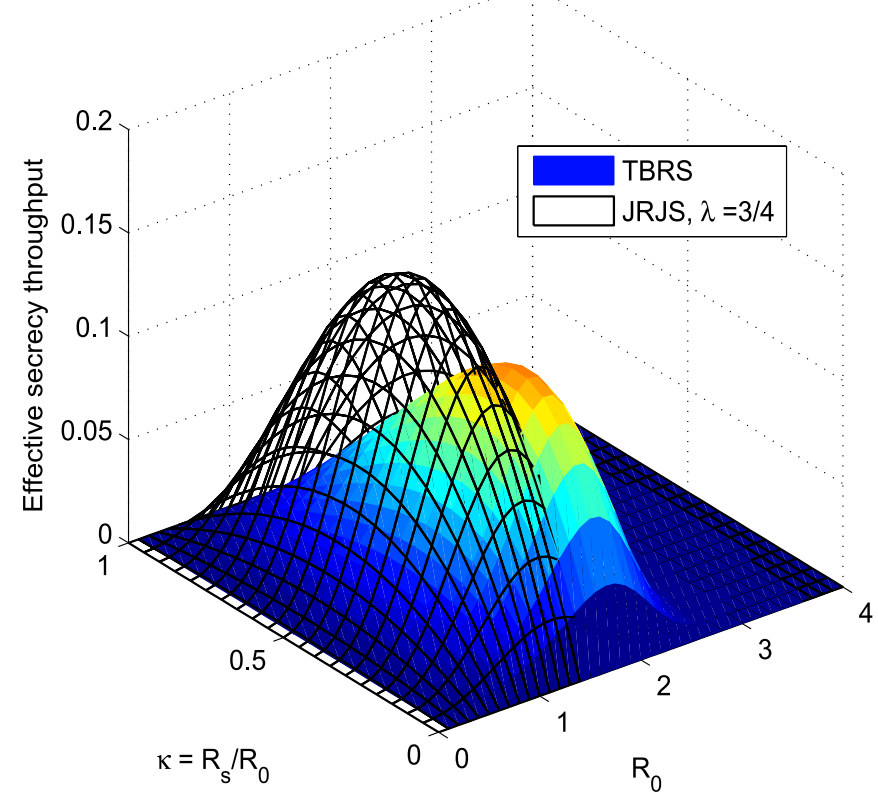

Fig. 8. Secrecy throughput versus $R_{0}$ and $\kappa=R_{s} / R_{0}$ for both the TBRS and JRJS strategies with $N_{t}=K_{r}=3, f_{d} T_{d}=0.1$, and $\eta=15 \mathrm{~dB}$.

To further evaluate the effect of feedback delays on the 726 secrecy performance, Fig. 7 plots the resultant percentage of 727 secrecy throughput loss versus the delay, which is defined as 728

$$
\varsigma_{\text {loss }}=\frac{\varsigma_{\text {no-delay }}-\varsigma_{\text {delay }}}{\varsigma_{\text {no-delay }}} \text {. }
$$

It can be seen from the figure that, compared with the TBRS 729 scheme, JRJS is more sensitive to the feedback delays. Further- 730 more, recalling that increasing the delay coefficient $\rho_{S R}$ of the 731 first hop improves the reliability, but at the same time also helps 732 the eavesdropper, it is not surprising that the secrecy throughput 733 loss due to the second-hop feedback delay is more pronounced. 734

Fig. 8 illustrates the achievable effective secrecy throughput 735 for both the TBRS and JRJS strategies versus the codeword 736 


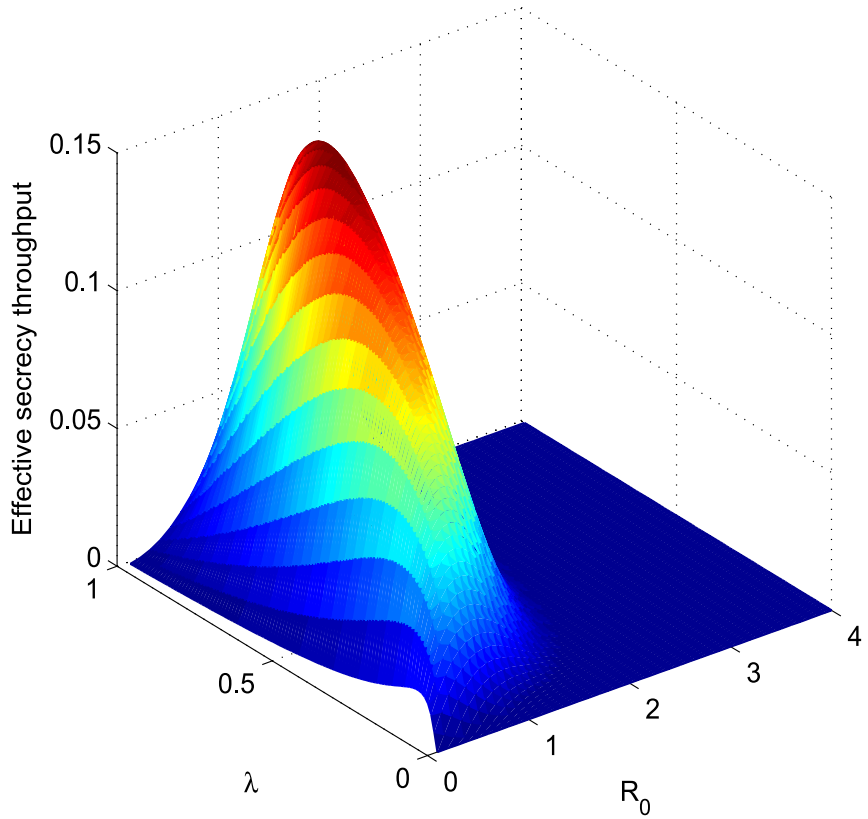

Fig. 9. Secrecy throughput versus $R_{0}$ and $\lambda$ for the JRJS strategy with $N_{t}=$ $K_{r}=3, f_{d} T_{d}=0.1, \eta=15 \mathrm{~dB}$, and $R_{s} / R_{0}=1 / 8$.

737 transmission rate $R_{0}$ and the secrecy code ratio $\kappa=R_{s} / R_{0}$ 738 with no outage constraints $(v=\delta=1)$. The values of the 739 effective secrecy throughput are plotted by using $\varsigma=R_{s} P_{R \& S}$. 740 We can observe in Fig. 8 that, subject to a fixed code rate 741 ratio $\kappa$, the effective secrecy throughput increases to a peak 742 value as $R_{0}$ reaches its optimal value and then decreases. This 743 phenomenon can be explained as follows. At a low transmission 744 rate, although the COP increases with $R_{0}$, which has a negative 745 effect on the effective secrecy throughput, both the secrecy 746 rate and the SOP performance will benefit. However, after 747 reaching the optimal $R_{0}$, the effective secrecy throughput drops 748 since the main link cannot afford a reliable transmission, and 749 the resultant COP increase becomes dominant. On the other 750 hand, subject to a fixed $R_{0}$ (which results in a constant COP), 751 the effective secrecy throughput is also a concave function 752 of $\kappa$, and increasing the code rate ratio ultimately results 753 in an increased secrecy information rate at the cost of an 754 increased SOP.

755 The achievable effective secrecy throughput for the JRJS 756 strategy is also presented in Fig. 8, and similar conclusions and 757 trends can be observed to that of the TBRS case. Additionally, 758 the comparison of the two strategies indicates that the JRJS 759 scheme attains a higher effective secrecy throughput than the 760 TBRS scheme operating without jamming, even if no power 761 sharing optimization has been employed.

762 Fig. 9 further illustrates the impact of power sharing between 763 the relay and the jammer on the achievable effective secrecy 764 throughput of the JRJS strategy versus $R_{0}$ in the absence of 765 outage constraints. Given a fixed code rate pair $\left(R_{0}, R_{s}\right)$, the 766 effective secrecy throughput follows the trend of the RSCP, 767 which is a concave function of $\lambda$, as shown in Fig. 6. The 768 interference introduced by the jammer initially improves both 769 the reliability and the security as $\lambda$ increases, but this trend is 770 reversed beyond a certain point.

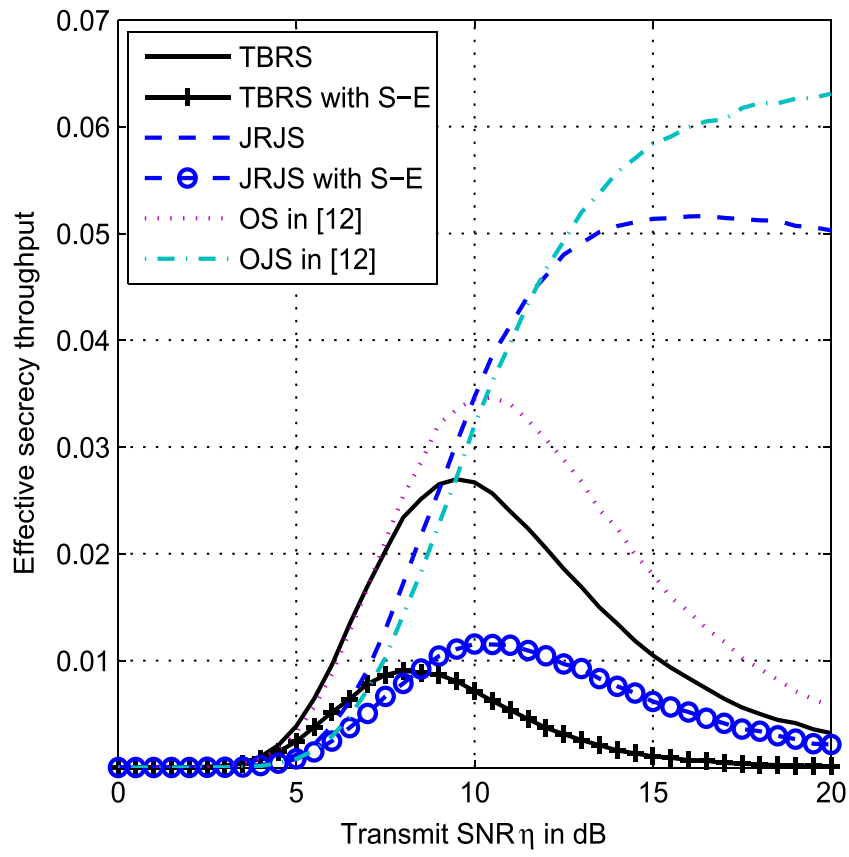

Fig. 10. Comparisons for different strategies with and without the S-E link, for $N_{t}=K_{r}=3, R_{0}=1, R_{s}=R_{0} / 8, f_{d} T_{d}=0.1$, and $\lambda=3 / 4$.

\section{DISCUSSION}

\section{A. Impact of the $S-E$ Link}

We note that the introduction of the S-E link, i.e., the 773 information leakage in the first phase, is very critical to the 774 security. There are also some research studies focusing on 775 the corresponding secure transmission design and performance 776 evaluation for cooperative networks with the S-E link, such 777 as [15] and [16]. Here, we assume that the eavesdropper can 778 receive information directly from the source in the first phase. 779 Thus, following the steps in the prior sections, for the TBRS 780 and JRJS schemes, it is clear that the SNR experienced at the 781 eavesdropper should be rewritten as

$$
\tilde{\gamma}_{E}^{\tau}=\gamma_{S E}+\gamma_{E}^{\tau}
$$

where $\gamma_{S E}=P_{s}\left|\mathbf{w}_{\text {opt }}\left(t \mid T_{d_{S R}}\right) \mathbf{h}_{S E}(t)\right|^{2} / N_{0}$ follows the ex- 783 ponential distribution with the average value $\bar{\gamma}_{S E}, \tau=784$ \{TBRS, JRJS \}, and $\gamma_{E}^{\tau}$ has been defined in (4) and (7). $\quad 785$

Then, the corresponding SOP, RSCP, and effective secrecy 786 throughput have to be reconsidered. Unfortunately, to the best 787 of our knowledge, it is a mathematically intractable problem 788 to obtain closed-form results for the related performance eval- 789 uations. Therefore, we resorted to numerical simulations for 790 further investigating the impact of the S-E link. Fig. $10 \mathrm{com}-791$ pares the effective secrecy throughput of the TBRS and JRJS 792 schemes both with and without considering the direct S-E 793 link. It becomes clear that the information leakage in the first 794 phase will lead to a severe security performance degradation, 795 particularly for the JRJS scheme, which will no longer be 796 capable of maintaining a steady throughput at high SNRs. The 797 reason for this trend is that increasing the transmit SNR will 798 help the eavesdropper in the presence of the direct S-E link. 


\section{B. Comparisons}

801 Here, based on the outdated CSI assumption, we provide per802 formance comparisons with a range of other schemes advocated 803 in [12] with the aid of the proposed outage-based characteriza804 tion. Fig. 10 also incorporates our effective secrecy throughput 805 performance comparison, where the optimal selection (OS) 806 regime and the optimal selection combined with jamming (OSJ) 807 were proposed in [12]. They are formulated as

$$
\begin{aligned}
& \text { OS }: R^{*}=\arg \max _{R_{k} \in \mathcal{R}}\left\{\frac{\tilde{\gamma}_{R_{k} D}}{\tilde{\gamma}_{R_{k} E}}\right\} \\
& \text { OSJ : }\left\{\begin{array}{l}
R^{*}=\arg \max _{R_{k} \in \mathcal{R}}\left\{\frac{\tilde{\gamma}_{R_{k} D}}{\tilde{\gamma}_{R_{k}} E}\right\} \\
J^{*}=\arg \min _{R_{k} \in \mathcal{R}-R^{*}}\left\{\frac{\tilde{\gamma}_{R_{k} D}}{\tilde{\gamma}_{R_{k} E}}\right\}
\end{array}\right.
\end{aligned}
$$

808 where $\tilde{\gamma}_{R_{k} E}$ is the delayed version of the instantaneous CSI of 809 the R-E link. It should be noted that this constitutes an entirely 810 new performance characterization of these schemes from the 811 perspective of the effective secrecy throughput. It is shown in 812 Fig. 1 that the selection combined with jamming outperforms 813 the corresponding nonjamming techniques at high SNRs, albeit 814 this trend may no longer prevail at low SNRs. In comparison, 815 compared with those selections relying on the average SNRs of 816 the R-E link, the optimal selections relying on the idealized 817 simplifying assumptions of having global CSI (OS and OSJ 818 schemes) knowledge can only achieve throughput gains at high 819 SNRs due to the inevitable feedback delay.

\section{CONCLUSION}

821 An outage-based characterization of cooperative relay net822 works has been provided in the face of CSI feedback delays. 823 Two types of relaying strategies were considered, namely, the 824 TBRS strategy and the JRJS strategy. Closed-form expressions 825 of the COP, the SOP, and the RSCP, as well as of the RSR, 826 were derived. The RSR results demonstrated that the reliability 827 is improved more substantially than the security performance 828 when the CSI feedback delays are reduced. Furthermore, we 829 presented a modified effective secrecy throughput definition 830 and demonstrated that the JRJS strategy achieves a significant 831 effective secrecy throughput gain over the TBRS strategy. The 832 transmit SNR, the secrecy codeword rate setting, and the power 833 sharing ratio between the relay and jammer nodes play impor834 tant roles in striking a balance between the reliability and the 835 security in terms of the secrecy throughput. The impact of the 836 direct S-E link and the performance comparisons with other 837 selection schemes were also included. Additionally, our results 838 demonstrate that JRJS is more sensitive to the feedback delays 839 and that the secrecy throughput loss due to the second-hop 840 feedback delay is more pronounced than that due to the first841 hop one.
842

843
APPENDIX A

Proof of PROPOSITION 1
844 To simplify the asymptotic performance analysis, (3) can be 845 expressed in a more mathematically tractable form by the com846 monly used tight upper bound of $\gamma_{D}^{\mathrm{TBRS}} \leq \min \left\{\gamma_{S R}, \gamma_{R^{*} D}\right\}$ and $\gamma_{E}^{\mathrm{TBRS}} \leq \min \left\{\gamma_{S R}, \gamma_{R^{*} E}\right\}$. When we have $\eta \rightarrow \infty$, based 847 on the CDFs in (9) and (10) and closing the smallest order terms 848 of $x / \eta$, we have

$$
\begin{aligned}
F_{\gamma_{S R}}(x) \rightarrow 1- & {\left[\sum_{n=0}^{N_{t}-1}\left(\begin{array}{c}
N_{t}-1 \\
n
\end{array}\right) \rho_{S R}^{2\left(N_{t}-1-n\right)}\left(1-\rho_{S R}^{2}\right)^{n}\right.} \\
& +\sum_{n=0}^{N_{t}-2}\left(\begin{array}{c}
N_{t}-1 \\
n
\end{array}\right) \times \rho_{S R}^{2\left(N_{t}-1-n\right)} \\
& \left.\times\left(1-\rho_{S R}^{2}\right)^{n} \frac{x}{\bar{\gamma}_{S R}}+\mathcal{O}\left(\frac{x}{\bar{\gamma}_{S R}}\right)\right] \\
\times & {\left[1-\frac{x}{\bar{\gamma}_{S R}}+\mathcal{O}\left(\frac{x}{\bar{\gamma}_{S R}}\right)\right] } \\
=1- & {\left[1+\left(1-\left(1-\rho_{S R}^{2}\right)^{N_{t}-1}\right) \frac{x}{\bar{\gamma}_{S R}}+\mathcal{O}\left(\frac{x}{\bar{\gamma}_{S R}}\right)\right] } \\
\times & {\left[1-\frac{x}{\bar{\gamma}_{S R}}+\mathcal{O}\left(\frac{x}{\bar{\gamma}_{S R}}\right)\right] } \\
= & \left(1-\rho_{S R}^{2}\right)^{N_{t}-1} \frac{x}{\bar{\gamma}_{S R}+\mathcal{O}\left(\frac{x}{\bar{\gamma}_{S R}}\right)}
\end{aligned}
$$

where $\mathcal{O}(x)$ denotes the high-order infinitely small contribu- 850 tions as a function of $x$, and

$$
\begin{aligned}
F_{\gamma_{R^{*}}}(x) \rightarrow 1 & -\sum_{k=0}^{K_{r}-1}(-1)^{k} \frac{K_{r}}{k+1}\left(\begin{array}{c}
K_{r}-1 \\
k
\end{array}\right) \\
& \times\left[1-\frac{k+1}{k\left(1-\rho_{R D}^{2}\right)+1} \frac{x}{\bar{\gamma}_{R D}}+\mathcal{O}\left(\frac{x}{\bar{\gamma}_{R D}}\right)\right] \\
= & \sum_{k=0}^{K_{r}-1}(-1)^{k}\left(\begin{array}{c}
K_{r}-1 \\
k
\end{array}\right) \frac{K_{r}}{k\left(1-\rho_{R D}^{2}\right)+1} \\
& \times \frac{x}{\bar{\gamma}_{R D}}+\mathcal{O}\left(\frac{x}{\bar{\gamma}_{R D}}\right) .
\end{aligned}
$$

Then, applying the upper bound of the receiver SNR, we may 852 rewrite the COP and the SOP of the TBRS strategy at high 853 SNRs as

$$
\begin{aligned}
P_{\mathrm{co}}^{\mathrm{TBRS}, \infty}= & 1-\left(1-F_{\gamma_{S R^{*}}}\left(\gamma_{t h}^{D}\right)\right)\left(1-F_{\gamma_{R^{*} D}}\left(\gamma_{t h}^{D}\right)\right) \\
= & {\left[\frac{\left(1-\rho_{S R}^{2}\right)^{N_{t}-1}}{\sigma_{S R}^{2}}+\sum_{k=0}^{K_{r}-1}(-1)^{k}\right.} \\
& \left.\times\left(\begin{array}{c}
K_{r}-1 \\
k
\end{array}\right) \frac{K_{r}}{\left[k\left(1-\rho_{R D}^{2}\right)+1\right] \sigma_{R D}^{2}}\right] \frac{2^{2 R_{0}}-1}{\eta}
\end{aligned}
$$

and according to the fact that $\gamma_{R^{*} E}$ is exponentially distributed, 855 we have

$$
\begin{aligned}
1-P_{\mathrm{so}}^{\mathrm{TBRS}, \infty} & =1-\left(1-F_{\gamma_{S R^{*}}}\left(\gamma_{t h}^{E}\right)\right)\left(1-F_{\gamma_{R^{*}}}\left(\gamma_{t h}^{E}\right)\right) \\
& =\left[\frac{\left(1-\rho_{S R}^{2}\right)^{N_{t}-1}}{\sigma_{S R}^{2}}+\frac{1}{\sigma_{R E}^{2}}\right] \frac{2^{2\left(R_{0}-R_{s}\right)}-1}{\eta} .
\end{aligned}
$$

Finally, substituting (41) and (42) into the definition of RSR 857 in (16), we can obtain (17). 


\section{APPENDIX B}

861 According to the description of COP and SOP, replacing $862 F_{\gamma_{R^{*} D}}(x)$ and $F_{\gamma_{R^{*} E}}(x)$ by $F_{\xi_{D}}(x)$ and $F_{\xi_{E}}(x)$ in (12) and (14) 863 will involve a mathematically intractable integration of the form

$$
\Upsilon(a, b, \mu, \nu)=\int_{0}^{\infty} \frac{z^{a}}{z+b} \exp \left(-\mu z-\frac{\nu}{z}\right) d z
$$

864 which, to the best of our knowledge, does not have a closed865 form solution. Alternatively, bearing in mind that the preceding 866 integration has a great matter with $\xi_{D}$, we now focus our 867 attention on the approximation of $\xi_{D}$. Based on the PDF 868 results in (23), it may be seen that $\gamma_{J^{*} D}$ obeys an exponential 869 distribution. Then, we can approximate $\hat{\gamma}_{J^{*} D}=\gamma_{J^{*} D}+1$ by 870 the exponential distribution as well, with an average value 871 of $\mathbb{E}\left\{\hat{\gamma}_{J^{*} D}\right\}=\left(\left[\left(K_{r}-1\right)\left(1-\rho_{R D}^{2}\right)+1\right] \bar{\gamma}_{R D}+K_{r}\right) / K_{r}$ by 872 assuming that the AWGN term " 1 " is part of the stochastic 873 mean terms. The approximation based on this method provides 874 a very accurate analysis, and the accuracy of this method is 875 verified by the numerical results of [34]. Thus, the CDF of $876 \hat{\xi}_{D}=\gamma_{R^{*} D} / \hat{\gamma}_{J^{*} D}$ can be derived as

$$
F_{\hat{\xi}_{D}}(x)=\sum_{k=0}^{K_{r}-1}(-1)^{k}\left(\begin{array}{c}
K_{r}-1 \\
k
\end{array}\right) \frac{K_{r}}{k+1} \frac{x}{x+\hat{\varphi}_{k}}
$$

877 where $\hat{\varphi}_{k}=\mathbb{E}\left\{\gamma_{R^{*} D}\right\} \mathbb{E} /\left\{\hat{\gamma}_{J^{*} D}\right\}$.

878 Then, substituting (44) into (11), we have

$$
\begin{aligned}
& F_{\gamma_{D}^{\mathrm{JRJS}}}(x) \\
& \approx \sum_{n=0}^{N_{t}-1} \sum_{k=0}^{K_{r}-1} \sum_{m=0}^{N_{t}-1-n}\left(\begin{array}{c}
N_{t}-1 \\
n
\end{array}\right)\left(\begin{array}{c}
K_{r}-1 \\
k
\end{array}\right)\left(\begin{array}{c}
N_{t}-1-n \\
m
\end{array}\right) \\
& \quad \times \frac{(-1)^{k} K_{r} \rho_{S R}^{2\left(N_{t}-1-n\right)}\left(1-\rho_{S R}^{2}\right)^{n} \varphi_{k} x^{N_{t}-1-n-m} e^{-\frac{x}{\gamma_{S R}}}}{\left(N_{t}-1-n\right) !(k+1) \bar{\gamma}_{S R}^{N_{t}-n}\left(x+\varphi_{k}\right)} \\
& \quad \times \int_{0}^{\infty} \frac{z^{m+1}}{z+\frac{x(x+1)}{x+\varphi_{k}}} \exp \left(-\frac{z}{\bar{\gamma}_{S R}}\right) d z .
\end{aligned}
$$

879 Using [33, eq. (3.383.10)], we can obtain the CDF of $\gamma_{D}^{\text {JRJS }}$ as

$$
\begin{aligned}
F_{\gamma_{D}^{\mathrm{JRJS}}}(x) \approx & 1-\sum_{n=0}^{N_{t}-1} \sum_{k=0}^{K_{r}-1} \sum_{m=0}^{N_{t}-1-n}\left(\begin{array}{c}
N_{t}-1 \\
n
\end{array}\right) \\
& \times\left(\begin{array}{c}
K_{r}-1 \\
k
\end{array}\right)\left(\begin{array}{c}
N_{t}-1-n \\
m
\end{array}\right) \\
& \times \frac{(-1)^{k}\left(K_{r}+1\right) \rho_{S R}^{2\left(N_{t}-1-n\right)}\left(1-\rho_{S R}^{2}\right)^{n}}{\left(N_{t}-1-n\right) !(k+1) \bar{\gamma}_{S R}^{N_{t}-n}} \\
& \times \frac{\Gamma(m+2) \hat{\varphi}_{k} x^{N_{t}-n}(x+1)^{m+1}}{\left(x+\hat{\varphi}_{k}\right)^{m+2}} \\
& \times \exp \left[-\frac{x\left(\hat{\varphi}_{k}-1\right)}{\bar{\gamma}_{S R}\left(x+\hat{\varphi}_{k}\right)}\right] \\
& \times \Gamma\left(-m-1, \frac{x(x+1)}{\bar{\gamma}_{S R}\left(x+\hat{\varphi}_{k}\right)}\right) .
\end{aligned}
$$

As far as the SOP is considered, we exploit the commonly 881 used tight upper bound of $\gamma_{E}^{\mathrm{JRJS}} \geq(1 / 2) \min \left\{\gamma_{S R}, \xi_{E}\right\}$ to 882 calculate it, which may be rewritten as

$$
\begin{aligned}
P_{\mathrm{SO}}^{\mathrm{JRJS}} & \approx \operatorname{Pr}\left\{\frac{1}{2} \min \left\{\gamma_{S R}, \xi_{E}\right\}>\gamma_{t h}^{E}\right\} \\
& =\left[1-F_{\gamma_{S R}}\left(2 \gamma_{t h}^{E}\right)\right]\left[1-F_{\xi_{E}}\left(2 \gamma_{t h}^{E}\right)\right]
\end{aligned}
$$

Substituting (9) and (25) into (47), we obtain $P_{\text {so }}^{\text {JRJS }}$.

\section{APPENDIX C}

\section{PROOF OF LEMMA 3}

According to the definition of the RSCP in (18), we can 887 calculate it by

888

$$
\begin{aligned}
& P_{R S}^{\mathrm{JRJS}}=\int_{0}^{\infty}\left[1-F_{\xi_{D}}\left(\gamma_{t h}^{D}+\frac{\gamma_{t h}^{D}\left(\gamma_{t h}^{D}+1\right)}{z}\right)\right] \\
& \quad \times F_{\xi_{E}}\left(\gamma_{t h}^{E}+\frac{\gamma_{t h}^{E}\left(\gamma_{t h}^{E}+1\right)}{z+\gamma_{t h}^{D}-\gamma_{t h}^{E}}\right) f_{\gamma_{S R^{*}}}\left(z+\gamma_{t h}^{D}\right) d z
\end{aligned}
$$

To make the integration mathematically tractable, we invoke 889 a simple approximation for $F_{\xi_{E}}(x)$ by treating the AWGN term 890 "1" in $\xi_{E}=\gamma_{R^{*} E} /\left(\gamma_{J^{*} E}+1\right)$ as part of the stochastic mean 891 terms. Hence, we have

$$
F_{\xi_{E}}(x)=\frac{x}{x+\hat{\phi}}
$$

where $\hat{\phi}=\lambda \eta \sigma_{R E}^{2} /\left((1-\lambda) \eta \sigma_{R E}^{2}+1\right)$.

Then, replacing the corresponding CDFs of the second hop 894 with $F_{\hat{\xi}_{D}}(x)$ and $F_{\hat{\xi}_{E}}(x)$ in (26), the integration can be derived as 895

$$
\begin{aligned}
& P_{R S}^{\mathrm{JRJS}} \approx 1-\sum_{n=0}^{N_{t}-1} \sum_{k=0}^{K_{r}-1} \sum_{m=0}^{N_{t}-1-n}(-1)^{k}\left(\begin{array}{c}
N_{t}-1 \\
n
\end{array}\right) \\
& \times\left(\begin{array}{c}
K_{r}-1 \\
k
\end{array}\right)\left(\begin{array}{c}
N_{t}-1-n \\
m
\end{array}\right) \\
& \times \frac{\left(K_{r}+1\right) \rho_{S R}^{2\left(N_{t}-1-n\right)}\left(1-\rho_{S R}^{2}\right)^{n}}{\left(N_{t}-1-n\right) !(k+1) \bar{\gamma}_{S R}^{N_{t}-n}} \\
& \times \frac{\hat{\varphi}_{k}\left(\gamma_{t h}^{D}\right)^{N_{t}-1-n-m}}{\gamma_{t h}^{D}+\hat{\varphi}_{k}} \exp \left(-\frac{\gamma_{t h}^{D}}{\bar{\gamma}_{S R}}-\frac{\gamma_{t h}^{D}}{\omega_{k} \bar{\gamma}_{R D}}\right) \\
& \times \int_{0}^{\infty} e^{\frac{-z}{\bar{\gamma}_{S R}} z^{m+1}}\left[\frac{1}{z+\theta_{1, k}}-\frac{\hat{\phi}\left(z+\gamma_{t h}^{D}-\gamma_{t h}^{E}\right) e^{\frac{-\gamma_{t h}^{E}}{\bar{\gamma}_{R E}}}}{\left(\gamma_{t h}^{E}+\hat{\phi}\right)\left(\theta_{1, k}-\theta_{2}\right)}\right. \\
&\left.\times\left(\frac{1}{z+\theta_{2}}-\frac{1}{z+\theta_{1, k}}\right)\right] d z
\end{aligned}
$$

where $\hat{\varphi}_{k}$ and $\hat{\phi}$ are introduced by relying on the similar approx- 896 imation as in Appendix B. Then, using [33, eq. (3.383.10)], we 897 obtain $P_{R \& S}^{J R J S}$. 


\section{REFERENCES}

1] B. Schneier, "Cryptographic design vulnerabilities," Computer, vol. 31, no. 9, pp. 29-33, Sep. 1998.

2] A. D. Wyner, "The wire-tap channel," Bell Syst. Techn. J., vol. 54, no. 8, pp. 1355-1387, Oct. 1975.

3] I. Csiszar and J. Korner, "Broadcast channels with confidential messages," IEEE Trans. Inf. Theory, vol. IT-24, no. 3, pp. 339-348, May 1978.

[4] W. K. Harrison, J. Almeida, M. R. Bloch, S. W. McLaughlin, and J. Barros, "Coding for secrecy: An overview of error-control coding techniques for physical-layer security," IEEE Signal Process. Mag., vol. 30, no. 5, pp. 41-50, Sep. 2013.

5] P. K. Gopala, L. Lai, and H. E. Gamal, "On the secrecy capacity of fading channels," IEEE Trans. Inf. Theory, vol. 54, no. 10, pp. 4687-4698, Oct. 2008.

[6] Y. W. P. Hong, P. C. Lan, and C. C. J. Kuo, "Enhancing physical-layer secrecy in multi-antenna wireless systems: An overview of signal processing approaches," IEEE Signal Process. Mag., vol. 30, no. 5, pp. 29-40, Sep. 2013.

[7] R. Bassily et al., "Cooperative security at the physical layer: A summary of recent advances," IEEE Signal Process. Mag., vol. 30, no. 5, pp. 16-28, Sep. 2013.

[8] L. Dong, Z. Han, A. P. Petropulu, and H. V. Poor, "Improving wireless physical layer security via cooperating relays," IEEE Trans. Signal Process., vol. 58, no. 3, pp. 1875-1888, Mar. 2010.

[9] J. Huang and A. L. Swindlehurst, "Cooperative jamming for secure communications in MIMO relay networks," IEEE Trans. Signal Process., vol. 59, no. 10, pp. 4871-4884, Oct. 2011.

0] Y.Zou, X. Wang, and W. Shen, "Optimal relay selection for physical-layer security in cooperative wireless networks," IEEE J. Sel. Areas Commun., vol. 31, no. 10, pp. 2099-2111, Oct. 2013.

11] Y. Zou, X. Wang, W. Shen, and L. Hanzo, "Security versus reliability analysis of opportunistic relaying," IEEE Trans. Veh. Technol., vol. 63, no. 6, pp. 2653-2661, Jul. 2014

2] I. Krikidis, J. S. Thompson, and S. McLaughlin, "Relay selection for secure cooperative networks with jamming," IEEE Trans. Wireless Commun., vol. 8, no. 10, pp. 5003-5011, Oct. 2009.

3] J. Chen, R. Zhang, L. Song, Z. Han, and B. Jiao, "Joint relay and jammer selection for secure two-way relay networks," IEEE Trans. Inf. Forensic Security, vol. 7, no. 1, pp. 310-320, Feb. 2012.

14] Z. Ding, M. Xu, J. Lu, and F. Liu, "Improving wireless security for bidirectional communication scenarios," IEEE Trans. Veh. Technol., vol. 61, no. 6, pp. 2842-2848, Jul. 2012.

5] C. Wang, H. M. Wang, and X. G. Xia, "Hybrid opportunistic relaying and jamming with power allocation for secure cooperative networks," IEEE Trans. Wireless Commun., vol. 14, no. 2, pp. 589-605, Feb. 2015.

16] H. Deng, H. M. Wang, W. Guo, and W. Wang, "Secrecy transmission with a helper: To relay or to jam," IEEE Trans. Inf. Forensic Security, vol. 10, no. 2, pp. 293-307, Feb. 2015.

7] B. He, X. Zhou, and T. D. Abhayapala, "Wireless physical layer security with imperfect channel state information: A survey," ZTE Commun., vol. 11, no. 3, pp. 11-19, Sep. 2013.

18] A. Mukherjee and A. L. Swindlehurst, "Robust beamforming for security in MIMO wiretap channels with imperfect CSI," IEEE Trans. Signal Process., vol. 59, no. 1, pp. 351-361, Jan. 2011.

19] J. Zhang and M. C. Gursoy, "Relay beamforming strategies for physicallayer security," in Proc. CISS, Princeton, NJ, USA, Mar. 2010, pp. 1-6.

20] M. Bloch, J. Barros, M. R. D. Rodrigues, and S. W. McLaughlin, "Wireless information-theoretic security," IEEE Trans. Inf. Theory, vol. 54, no. 6, pp. 2515-2534, Jun. 2008.

21] X. Zhou, M. R. McKay, B. Maham, and A. Hjorungnes, "Rethinking the secrecy outage formulation: A secure transmission design perspective," IEEE Commun. Lett., vol. 15, no. 3, pp. 302-304, Mar. 2011.

22] J. Hu, Y. Cai, N. Yang, and W. Yang, "A new secure transmission scheme with outdated antenna selection," IEEE Trans. Inf. Forensics Security, to be published.

23] J. Hu, W. Yang, N. Yang, X. Zhou, and Y. Cai, "On-off-based secure transmission design with outdated channel state information," IEEE Trans. Veh. Technol., to be published.

24] N. E. Wu and H. J. Li, "Effect of feedback delay on secure cooperative networks with joint relay and jammer selection," IEEE Wireless Commun. Lett., vol. 2, no. 4, pp. 415-418, Aug. 2013.

25] X. Guan Y. Cai and Y. Yang, "Secure transmission design and performance analysis for cooperation exploring outdated CSI," IEEE Commun. Lett., vol. 18, no. 9, pp. 1637-1640, Sep. 2014.
[26] L. Wang, S. Xu, W. Yang, W. Yang, and Y. Cai, "Security performance 975 of multiple antennas multiple relaying networks with outdated relay 976 selection," in Proc. WCSP, Hefei, China, Oct. 2014, pp. 1-6.

[27] J. Huang and A. L. Swindlehurst, "Buffer-aided relaying for two-hop 978 secure communication," IEEE Trans. Wireless Commun., vol. 14, no. 1, 979 pp. 152-164, Jan. 2015.

[28] S. I. Kim, I. M. Kim, and J. Heo, "Secure transmission for multiuser relay 981 networks," IEEE Trans. Wireless Commun., vol. 14, no. 7, pp. 3724-3737, 982 Jul. 2015.

[29] Y. Ma, D. Zhang, A. Leith, and Z. Wang, "Error performance of transmit 984 beamforming with delayed and limited feedback," IEEE Trans. Wireless 985 Commun., vol. 8, no. 3, pp. 1164-1170, Mar. 2009.

[30] Z. Rezki, A. Khisti, and M. S. Alouini, "Ergodic secret message capac- 987 ity of the wirechannel with finite-rate feedback," IEEE Trans. Wireless 988 Commun., vol. 13, no. 6, pp. 3364-3379, Jun. 2014.

[31] X. Tang, R. Liu, P. Spasojevic, and H. V. Poor, "On the throughput of 990 secure hybrid-ARQ protocols for Gaussian block-fading channels," IEEE 991 Trans. Inf. Theory, vol. 55, no. 4, pp. 1575-1591, Apr. 2009.

[32] H. A. Suraweera, M. Soysa, C. Tellambura, and H. K. Garg, "Performance 993 analysis of partial relay selection with feedback delay," IEEE Signal 994 Process. Lett., vol. 17, no. 6, pp. 531-534, Jun. 2010.

[33] I. S. Gradshteyn and I. M. Ryzhik, Table of Integrals, Series and Products, 996 6th ed. San Diego, CA, USA: Academic, 2000.

[34] S. Kim and J. Heo. "Outage probability of interference-limited amplify- 998 and-forward relaying with partial relay selection," in Proc. IEEE VTC, 999 Yokohama, Japan, May 2011, pp. 1-5.

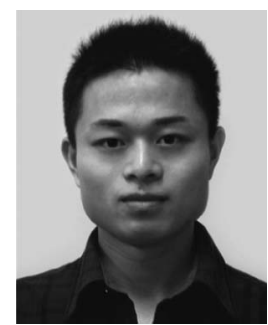
tions, and physical layer security
Lei Wang (S'11) received the B.S. degree in elec- 1001 tronics and information engineering from Central 1002 South University, Changsha, China, in 2004 and 1003 the M.S. degree in communications and informa- 1004 tion systems from PLA University of Science and 1005 Technology, Nanjing, China, in 2011. He is currently 1006 working toward the Ph.D. degree in communications 1007 and information systems with PLA University of 1008 Science and Technology.

1009

His current research interests include cooperative 1010 communications, signal processing in communica- 1011 1012

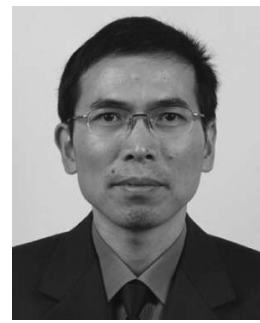

Yueming Cai (M'05-SM'12) received the B.S. 1013 degree in physics from Xiamen University, 1014 Xiamen, China, in 1982 and the M.S. degree in 1015 microelectronics engineering and the Ph.D. degree in 1016 communications and information systems from 1017 Southeast University, Nanjing, China, in 1988 and 1018 1996, respectively.

1019

He is currently with the College of Communica- 1020 AQ5 tions Engineering, PLA University of Science and 1021 Technology, Nanjing, China. His current research 1022 interests include multiple-input-multiple-output sys- 1023 tems, orthogonal frequency-division multiplexing systems, signal processing in 1024 communications, cooperative communications, and wireless sensor networks. 1025 


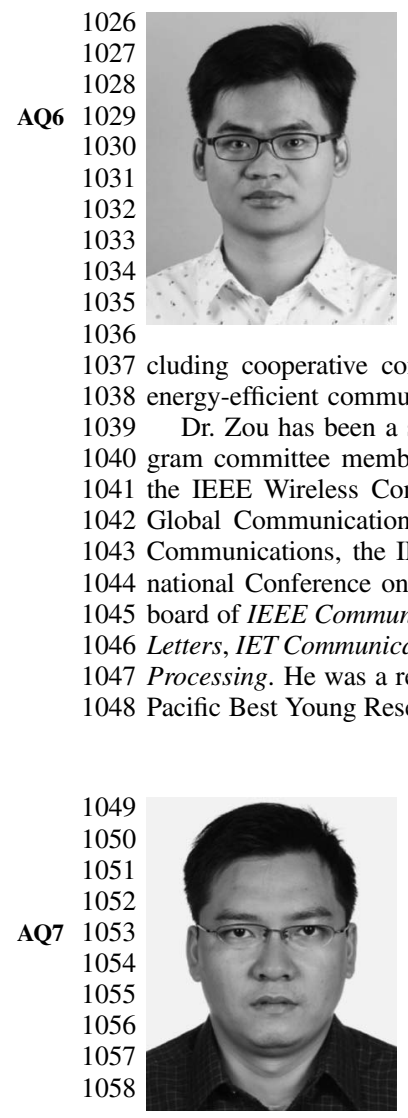

Yulong Zou (SM'13) received the B.Eng. degree in information engineering from Nanjing University of Posts and Telecommunications (NUPT), Nanjing, China, in July 2006; the Ph.D. degree in electrical engineering from Stevens Institute of Technology, Hoboken, NJ, USA, in May 2012; and the Ph.D. degree in signal and information processing from NUPT in July 2012.

$\mathrm{He}$ is currently a Professor with NUPT. His research interests span a wide range of topics in wireless communications and signal processing, in-
munications, cognitive radio, wireless security, and 1038 energy-efficient communications.

1039 Dr. Zou has been a symposium chair, a session chair, and a technical program committee member for several IEEE-sponsored conferences, including 041 the IEEE Wireless Communications and Networking Conference, the IEEE Global Communications Conference, the IEEE International Conference on nications, the IEEE Vehicular Technology Conference, and the InterCommunications Surveys and Tutorials, IEEE Communication 047 Processing. He was a received the 2014 IEEE Communications Society Asia048 Pacific Best Young Researcher award.

Weiwei Yang (S'08-M'12) received the B.S., M.S., and Ph.D. degrees from PLA University of Science and Technology, Nanjing, China, in 2003, 2006, and 2011, respectively.

$\mathrm{He}$ is currently with the College of Communications Engineering, PLA University of Science and Technology. His research interests are orthogonal frequency-domain multiplexing systems, signal processing in communications, cooperative communications, cognitive networks, and network security.

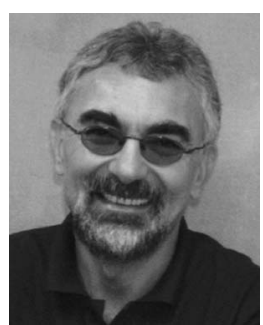

Lajos Hanzo (M'91-SM'92-F'04) received the 1059 M.S. degree in electronics and the Ph.D. de- 1060 gree from the Technical University of Budapest, 1061 Budapest, Hungary, in 1976 and 1983, respectively; 1062 the D.Sc. degree from the University of Southampton, 1063 Southampton, U.K., in 2004; and the "Doctor Honoris 1064 Causa" degree from the Technical University of 1065 Budapest in 2009. 1066

During his 38-year career in telecommunications, 1067 he has held various research and academic posts in 1068 Hungary, Germany, and the U.K. Since 1986, he has 1069 been with the School of Electronics and Computer Science, University of 1070 Southampton, where he holds the Chair in Telecommunications. He is currently 1071 directing an academic research team, working on a range of research projects 1072 in the field of wireless multimedia communications sponsored by industry, the 1073 Engineering and Physical Sciences Research Council (EPSRC), the European 1074 Research Council's Advanced Fellow Grant, and the Royal Society's Wolfson 1075 Research Merit Award. During 2008-2012, he was a Chaired Professor with 1076 Tsinghua University, Beijing, China. He is an enthusiastic supporter of in- 1077 dustrial and academic liaison and offers a range of industrial courses. He 1078 has successfully supervised about 100 Ph.D. students, coauthored 20 John 1079 Wiley/IEEE Press books on mobile radio communications totaling in excess of 1080 10000 pages, and published more than 1400 research entries on IEEE Xplore. 1081

Dr. Hanzo is a Fellow of the Royal Academy of Engineering, the Institution 1082 of Engineering and Technology, and the European Association for Signal 1083 Processing. He is also a Governor of the IEEE Vehicular Technology Society. 1084 During 2008-2012, he was the Editor-in-Chief of IEEE Press. He has served 1085 as the Technical Program Committee Chair and the General Chair of IEEE 1086 conferences, has presented keynote lectures, and has received a number of 1087 distinctions. His published work has more than 20000 citations. Further in- 1088 formation on research in progress and associated publications is available at 1089 http://www-mobile.ecs.soton.ac.uk. 


\section{AUTHOR QUERIES}

\section{AUTHOR PLEASE ANSWER ALL QUERIES}

AQ1 = RV was expanded as "random variable." Please check if appropriate. Otherwise, please make the necessary changes.

AQ2 = Equations (29) and (30) are missing in the document. Please check.

AQ3 = Please provide publication update in Ref [22].

AQ4 = Please provide publication update in Ref [23].

AQ5 = Current affiliation of author Yueming Cai was provided as captured from the first footnote. Please check if appropriate. Otherwise, please make the necessary changes.

AQ6 $=$ Please confirm that Dr. Zou has received two Ph.D. degrees.

AQ7 = Current affiliation of author Weiwei Yang was provided as captured from the first footnote. Please check if appropriate. Otherwise, please make the necessary changes.

END OF ALL QUERIES 


\title{
Joint Relay and Jammer Selection Improves the Physical Layer Security in the Face of CSI Feedback Delays
}

\author{
Lei Wang, Student Member, IEEE, Yueming Cai, Senior Member, IEEE, Yulong Zou, Senior Member, IEEE, \\ Weiwei Yang, Member, IEEE, and Lajos Hanzo, Fellow, IEEE
}

6 Abstract-We enhance the physical layer security (PLS) of 7 amplify-and-forward (AF) relaying networks with the aid of joint 8 relay and jammer selection (JRJS), despite the deleterious effect 9 of channel state information (CSI) feedback delays. Furthermore, 10 we conceive a new outage-based characterization approach for the 11 JRJS scheme. The traditional best relay selection (TBRS) is also 12 considered as a benchmark. We first derive closed-form ex13 pressions of both the connection outage probability (COP) and 14 the secrecy outage probability (SOP) for both the TBRS and 15 JRJS schemes. Then, a reliable and secure connection probability 16 (RSCP) is defined and analyzed for characterizing the effect of 17 the correlation between the COP and the SOP introduced by the 18 corporate source-relay link. The reliability-security ratio (RSR) 19 is introduced for characterizing the relationship between the re20 liability and the security through asymptotic analysis. Moreover, 21 the concept of effective secrecy throughput is defined as the 22 product of the secrecy rate and of the RSCP for the sake of 23 characterizing the overall efficiency of the system, as determined 24 by the transmit SNR, the secrecy codeword rate, and the power 25 sharing ratio between the relay and the jammer. The impact of 26 the direct source-eavesdropper link and additional performance 27 comparisons with respect to other related selection schemes are 28 also included. Our numerical results show that the JRJS scheme 29 outperforms the TBRS method both in terms of the RSCP and in 30 terms of its effective secrecy throughput, but it is more sensitive to 31 the feedback delays. Increasing the transmit signal-to-noise ratio 32 (SNR) will not always improve the overall throughput. Moreover, 33 the RSR results demonstrate that, upon reducing the CSI feedback 34 delays, the reliability improves more substantially than the secu35 rity degrades, implying an overall improvement in terms of the 36 security-reliability tradeoff. Additionally, the secrecy throughput 37 loss due to the second-hop feedback delay is more pronounced 38 than that due to the first-hop one.

Manuscript received October 2, 2014; revised February 25, 2015 and July 27, 2015; accepted September 8, 2015. This work was supported in part by the National Natural Science Foundation of China under Grant 61371122, Grant 61471393, and Grant 61501512 and in part by the Natural Science Foundation of Jiangsu Province under Grant BK20150718 and Grant BK20150040. The review of this paper was coordinated by Prof. M. C. Gursoy.

L. Wang, Y. Cai, and W. Yang are with the College of Communications Engineering, PLA University of Science and Technology, Nanjing 210007, China (e-mail: csu-wl@163.com; caiym@vip.sina.com; yww_1010@aliyun.com).

Y. Zou is with the School of Telecommunications and Information Engineering, Nanjing University of Posts and Telecommunications, Nanjing 210003, China (e-mail: yulong.zou@njupt.edu.cn).

L. Hanzo is with the Department of Electronics and Computer Science, University of Southampton, Southampton SO17 1BJ, U.K. (e-mail: 1h@ecs. soton.ac.uk).

Color versions of one or more of the figures in this paper are available online at http://ieeexplore.ieee.org.

Digital Object Identifier 10.1109/TVT.2015.2478029
Index Terms-Effective secrecy throughput, feedback delay, 39 physical layer security (PLS), relay and jammer selection, 40 reliability and security.

\section{INTRODUCTION}

$\mathbf{W}$ IRELESS communications systems are particularly 43 vulnerable to security attacks because of the inherent 45 openness of the transmission medium. Traditionally, the infor- 46 mation privacy of wireless networks has been focused on the 47 higher layers of the protocol stack employing cryptographically 48 secure schemes. However, these methods typically assume a 49 limited computing power for the eavesdroppers and exhibit 50 inherent vulnerabilities in terms of the inevitable secret key 51 distribution and management [1]. In recent years, physical 52 layer security (PLS) has emerged as a promising technique of 53 improving the confidentiality wireless communications, which 54 exploits the time-varying properties of fading channels, instead 55 of relying on conventional cryptosystems. The pivotal idea of 56 PLS solutions is to exploit the dynamically fluctuating random 57 nature of radio channels for maximizing the uncertainty con- 58 cerning the source messages at the eavesdropper [2], [3].

59

To achieve this target, several PLS-enhancement approaches 60 have been proposed in the literature, including secrecy- 61 enhancing channel coding [4], secure on-off transmission de- 62 signs [5], secrecy-improving beamforming (BF)/precoding, and 63 artificial-noise-aided techniques relying on multiple antennas 64 [6], as well as secure relay-assisted transmission techniques [7]. 65 Specifically, apart from improving the reliability and coverage 66 of wireless transmissions, user cooperation also has a great 67 potential in terms of enhancing the wireless security against 68 eavesdropping attacks. There has been a growing interest in 69 improving the security of cooperative networks at the physical 70 layer [8]-[14]. To explore the spatial diversity potential of the 71 relaying networks and to boost the secrecy capacity (the differ- 72 ence between the channel capacity of the legitimate main link 73 and that of the eavesdropping link), most of the existing work 74 has been focused on secrecy-enhancing BF [8], [9], as well as 75 on intelligent relay node/jammer node (RN/JN) selection, etc. 76 Notably, given the availability of multiple relays, appropriately 77 designed $\mathrm{RN} / \mathrm{JN}$ selection is capable of achieving a signifi- 78 cant security improvement for cooperative networks, which is 79 emerging as a promising research topic. In particular, Zou et al. 80 investigated both amplify-and-forward (AF)- and decode-and- 81 forward (DF)-based optimal relay selection conceived for 82 
83 enhancing the PLS in cooperative wireless networks [10], [11], 84 where the global channel state information (CSI) of both the 85 main link and the eavesdropping link was assumed to be avail86 able. Similarly, jamming techniques, which impose artificial 87 interference on the eavesdropper, have also attracted substantial 88 attention [12]-[14]. More specifically, several sophisticated 89 joint relay and jammer selection (JRJS) schemes were proposed 90 in [12], where the beneficially selected relay increases the reli91 ability of the main link, whereas the carefully selected jammer 92 imposes interference on the eavesdropper and simultaneously 93 protects the legitimate destination from interference. In [13] 94 and [14], cooperative jamming has been studied in the context 95 of bidirectional scenarios, and efficient RN/JN selection criteria 96 have been developed for achieving improved secrecy rates with 97 the aid of multiple relays. Furthermore, more effective relaying 98 and jamming schemes, when taking the information leakage 99 of the source-eavesdropper link into consideration, have been 100 presented lately in [15] and [16].

101 Nevertheless, an idealized assumption of the previously re102 ported research on PLS is the availability of perfect channel 103 state information (CSI), which is regarded as a stumbling block 104 in the way of invoking practical secrecy-enhancing Wyner 105 coding, on-off design, BF/precoding, and RN/JN selection. 106 However, this idealized simplifying assumption is not realistic, 107 since practical channel estimation imposes CSI imperfections, 108 which are aggravated by the feedback delay, limited-rate feed109 back, and channel estimation errors (CEEs) [17]. Generally, the 110 related research has been focused on the issues of robust secure $111 \mathrm{BF}$ design from an average secrecy-rate-based optimization 112 perspective for point-to-point multiantenna aided channels and 113 relay channels [18], [19] supporting delay-tolerant systems. 114 For systems imposing stringent delay constraints, particularly 115 in imperfect CSI scenarios, perfect secrecy cannot always be 116 achieved. Hence, the secrecy-outage-based characterization of 117 systems is more appropriate, which provides a probabilistic 118 performance measure of secure communication. The concept 119 of secrecy outage was adopted in [20] for characterizing the 120 probability of having both reliable and secure transmission, 121 which, however, is inapplicable for the imperfect CSI case and 122 fails to distinguish a connection outage from the secrecy outage. 123 In [21], an alternative secrecy outage formulation is proposed 124 for characterizing the attainable security level and provided 125 a general framework for designing transmission schemes that 126 meet specific target security requirements. To quantify both the 127 reliability and security performance at both the legitimate and 128 eavesdropper nodes separately, two types of outages, namely, 129 the connection outage probability (COP) and the secrecy outage 130 probability (SOP) are introduced. Then, considering the impact 131 of time delay caused by the antenna selection process at the 132 legitimate receiver, $\mathrm{Hu}$ et al. [22] proposed a new secure 133 transmission scheme in the multiinput multioutput multieaves134 dropper wiretap channel. Much recently, considering the out135 dated CSI from the legitimate receiver, a new secure on-off 136 transmission scheme was proposed for enhancing the secrecy 137 throughput in [23].

138 Moreover, prior studies of the outage-based secure trans139 mission design are limited to single-antenna-assisted single140 hop systems and have not been considered for cooperative relaying systems. Hence, the issues of secure transmissions 141 over cooperative relaying channels expressed in terms of the 142 SOP, COP, and secrecy throughput constitute an open problem. 143 On the other hand, apart from CEE, the CSI feedback delay 144 results in critical challenges for the PLS of cooperative relaying 145 systems, particularly when considering the specifics of RN/JN 146 selection. In [15], the effects of outdated CSI knowledge con- 147 cerning the legitimate links on the ergodic secrecy rate achieved 148 by the proposed secure transmission strategy in the context 149 of DF relaying is investigated. The impact of CSI feedback 150 delay on the secure relay and jammer selection conceived for 151 DF relaying was investigated in [24], albeit only in terms 152 of the SOP. In our previous study [25], we considered the 153 secure transmission design and the secrecy performance of an 154 opportunistic DF system relying on outdated CSI, where only a 155 single relay is invoked. Additionally, during the revision of this 156 work, we investigated the security performance for outdated AF 157 relay selection in [26]. Therefore, in this treatise, we extend 158 our investigations to the PLS of multiple AF relaying assisted 159 networks relying on RN/JN selection.

Explicitly, we focus our attention on the outage-based char- 161 acterization of secure transmissions in cooperative relay-aided 162 networks relying on realistic CSI feedback delay. To exploit the 163 multirelay induced diversity gain and the associated jamming 164 capabilities, joint AF relay node and jammer node selection 165 is employed by the relay-destination link. We assume that, in 166 line with the practical reality, the instantaneous eavesdropper's 167 CSI is unavailable at the legitimate transmitter and that the 168 RN/JN selections are performed based on the outdated CSI of 169 the main links. Two types of cooperative strategies are invoked 170 by our cooperative network operating under secrecy constraints, 171 namely, the traditional best relay selection (TBRS) strategy and 172 the JRJS strategy. Specifically, the main contributions of this 173 paper can be summarized as follows.

- We develop an outage-based characterization for quan- 176 tifying both the reliability and security performance of 177 a two-hop AF relaying system. Specifically, in contrast 178 to [21] and [22], we propose the novel definition of 179 the reliable and secure connection probability (RSCP). 180 Explicitly, closed-form expressions of the COP, the SOP, 181 and the RSCP are derived for both the TBRS and for our 182 JRJS strategies. Numerical results demonstrate that the 183 JRJS scheme outperforms the TBRS scheme in terms of 184 its RSCP.

- We also introduce the reliability-security ratio (RSR) 186 for characterizing their direct relationship by a single 187 parameter through the asymptotic analysis of the COP and 188 the SOP in the high-SNR regime. We derive the RSR for 189 both the TBRS and JRJS strategies for investigating the 190 effect of secrecy codeword rate setting, as well as that 191 of the feedback delay and that of the power sharing ratio 192 between the relay and the jammer on the RSR.

- We then modify the definition of effective secrecy 194 throughput by multiplying the secrecy rate with the RSCP, 195 which results in an optimization problem of the trans- 196 mit signal-to-noise ratio (SNR), secrecy codeword rate, 197 and power sharing between the relay and the jammer. 198 


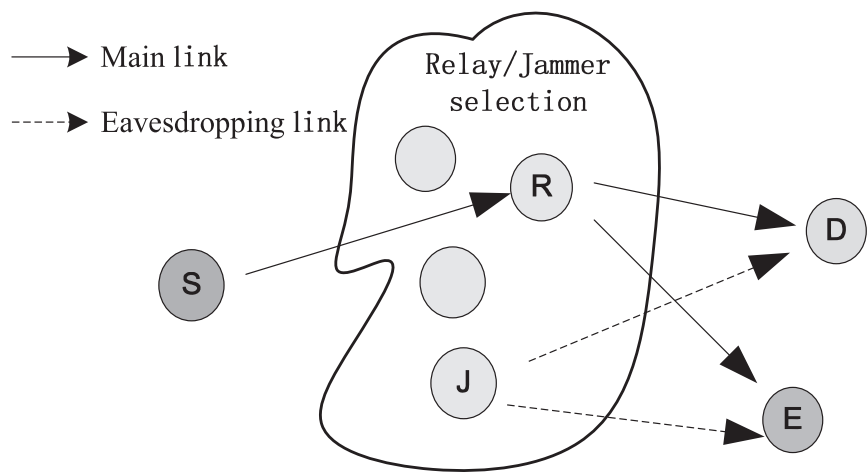

Fig. 1. Cooperative relaying network assisted by multiple relays in the presence of an eavesdropper.

206 The remainder of this paper is organized as follows. 207 Section II introduces our system model and describes both 208 the TBRS and our JRJS strategies. In Sections III and IV, 209 we present the mathematical framework of our performance 210 analysis both for the TBRS strategy and for the JRJS strategy, 211 respectively, including the COP, the SOP, the RSCP, the RSR, 212 and the effective secrecy throughput. Our numerical results 213 and discussions are provided in Section V. Finally, Section VI 214 presents our concluding remarks.

\section{SYSTEM MODEL}

\section{A. System Description}

217 Consider a cooperative relaying network consisting of a 218 source $S$, a destination $D, K_{r}$ relays $R_{k}, k=1, \ldots, K_{r}$, and 219 an eavesdropper $E$, as shown in Fig. 1, where all nodes are 220 equipped with a single transmit antenna (TA), except for the 221 source, which has $N_{t}$ TAs. The cooperative relay architecture 222 in Fig. 1 is generally applicable to diverse practical wireless 223 systems in the presence of an eavesdropper, including the 224 family of wireless sensor networks (WSNs), mobile ad hoc 225 networks (MANETs), and the long-term evolution advanced 226 cellular systems [11].

227 To exploit the diversity potential of multiple relay nodes over 228 independently fading channels, AF relay/jammer selection is 229 employed. All relays operate in the half-duplex AF mode, and 230 data transmission is performed in two phases. More particu231 larly, during the broadcast phase, the source node transmits its 232 signal to a selected relay with the aid of $\mathrm{BF}$, which is invoked 233 for forwarding the signal received from $S$ to $D$. An inherent 234 assumption is that the transmit BF weights are based on the 235 CSI estimates quantified and fed back by the selected relay. 236 During the cooperative phase, a pair of appropriately selected 237 relays transmit toward $D$ and $E$, respectively. A conventional relay (denoted by $R^{*}$ ) forwards the source's message to the 238 destination. Another relay (denoted by $J^{*}$ ) operates in the 239 "jammer mode" and imposes intentional interference upon $E$ in 240 order confuse it. However, $D$ is unable to mitigate the artificial 241 interference emanating from the jammer node $J^{*}$ due to its 242 critical secrecy constraints [12]. It should be noted that both 243 the process of RN/JN selection and the feedback of the transmit 244 BF weights from $R^{*}$ to $S$ may impose a time lag between the 245 data transmission and the channel estimation. These time delays 246 are denoted by $T_{d_{S R}}$ and $T_{d_{R D}}$, respectively. Furthermore, we 247 assume that the $\mathrm{BF}$ and $\mathrm{RN} / \mathrm{JN}$ selection process is based 248 on the perfectly estimated but outdated CSI. We employ the 249 first-order autoregressive outdated CSI model of [20], while 250 relying on the correlation coefficients of $\rho_{S R}=J_{0}\left(2 \pi f_{d} T_{d_{S R}}\right) 251$ and $\rho_{R D}=J_{0}\left(2 \pi f_{d} T_{d_{R D}}\right)$ for the two hops, where $J_{0}(\cdot)$ is 252 the zero-order Bessel function of the first kind, and $f_{d}$ is the 253 Doppler frequency.

A slow flat block Rayleigh fading environment is assumed, 255 where the channel remains static for the coherence interval (one 256 slot) and changes independently in different coherence inter- 257 vals, as denoted by $h_{i, j} \sim \mathcal{C N}\left(0, \sigma_{i, j}^{2}\right), i, j \in\{S, R, J, D, E\} .258$ The direct communication links are assumed to be unavailable 259 due to the presence of obstructions between $S$ and $D$, as well 260 as the eavesdropper. ${ }^{1}$ This assumption follows the rationale of 261 [12] and has been routinely exploited in previous literature (see 262 [27] and [28] and the references therein), where the source 263 and relays belong to the same cluster, whereas the destination 264 and the eavesdropper are located in another. More specifically, 265 this assumption is particularly valid in networks with broadcast 266 and unicast transmission, where each terminal is a legitimate 267 receiver for one signal and acts as an eavesdropper for some 268 other signal. Therefore, the security concerns are only related 269 to the cooperative relay-aided channel. Furthermore, additive 270 white Gaussian noise (AWGN) is assumed with zero mean 271 and unit variance $N_{0}$. Let $P_{i}$ be the transmit power of node 272 $i$, and the instantaneous SNR of the $i \rightarrow j$ link is given by 273 $\gamma_{i, j}=P_{i}\left|h_{i, j}\right|^{2} / N_{0}$.

We employ the constant-rate Wyner coding scheme for con- 275 structing wiretap codes of [2] to meet the PLS requirements 276 due to the fact that the accurate global CSI is not available. 277 Let $\mathbb{C}\left(R_{0}, R_{s}, N\right)$ denote the set of all possible Wyner codes 278 of length $N$, where $R_{0}$ is the codeword transmission rate, and 279 $R_{s}$ is the confidential information rate $\left(R_{0}>R_{s}\right)$. The positive 280 rate difference $R_{e}=R_{0}-R_{s}$ is the cost of providing secrecy 281 against the eavesdropper. A confidential message is encoded 282 into a codeword at $S$ and then transmitted to $D$.

\section{B. Secure Transmission}

In the broadcast phase, $S$ transmits its BF signal $s(t)$ to the 285 selected relay $R^{*}$, where the relay selection is performed 286 before data transmission commences, and the selection cri- 287 terion will be detailed later in the context of the cooper- 288 ative phase. The transmit BF vector $\mathbf{w}\left(t \mid T_{d}\right)$ is calculated 289 using the perfectly estimated but outdated CSI given by 290

\footnotetext{
${ }^{1}$ The case when the $S \rightarrow E$ link is introduced will be investigated separately in Section VI.
} 
$291 \mathbf{w}\left(t \mid T_{d_{S R}}\right)=\mathbf{h}_{S R^{*}}^{H}\left(t-T_{d}\right) /\left|\mathbf{h}_{S R^{*}}\left(t-T_{d_{S R}}\right)\right|$ [29], where we 292 have $\mathbf{h}_{S R^{*}}(t)=\left[h_{S R^{*}, 1}(t), \ldots, h_{S R^{*}, N_{t}}(t)\right]^{T}$, and the signal 293 received by the relay $R^{*}$ can be written as

$$
y_{R^{*}}(t)=\sqrt{P_{s}} \mathbf{w}\left(t \mid T_{d}\right) \mathbf{h}_{S R^{*}}(t) s(t)+n_{S R^{*}}(t)
$$

294 where $n_{S R^{*}}(t)$ is the AWGN at the relay. Then, we can 295 define the received $\mathrm{SNR}$ at the relay node as $\gamma_{S R}=$ $296 P_{S}\left|\mathbf{w}\left(t \mid T_{d_{S R}}\right) \mathbf{h}_{S R^{*}}(t)\right|^{2} / N_{0}$.

297 In the cooperative phase, we consider two RN/JN selection 298 schemes performed by $D$ : relay selection without jamming 299 and JRJS.

300 1) Traditional Best Relay Selection: The first category of so301 lutions does not involve a jamming process, and therefore, only 302 a conventional relay accesses the channel during the second 303 phase of the protocol. The relay selection process is performed 304 based on the highest instantaneous SNR of the second hop, 305 which is formulated as

$$
R^{*}=\arg \max _{R_{k} \in \mathcal{R}}\left\{\frac{\tilde{\gamma}_{R_{k} D}}{\mathbb{E}\left[\gamma_{R_{k} E}\right]}=\frac{P_{R}\left|\tilde{h}_{R_{k} D}\left(t-T_{d}\right)\right|^{2}}{N_{0} \mathbb{E}\left[\gamma_{R_{k} E}\right]}\right\}
$$

306 where $\tilde{\gamma}_{R_{k} D}$ is the instantaneous SNR in the relay selection 307 process, and $\mathbb{E}\left[\gamma_{R_{k} E}\right]$ denotes the average SNR at $E$. We can AQ1 308 model $\gamma_{R_{k} D}$ and $\tilde{\gamma}_{R_{k} D}$ as two gamma distributed random 309 variables having the correlation factor of $\rho_{R D}^{2}$.

310 During the second phase, the received signal $y_{R^{*}}(t)$ 311 is multiplied by a time-variant AF-relay gain $G$ and 312 retransmitted to $D$, where we have $G=$ $313 \sqrt{P_{R} /\left(P_{S}\left|\mathbf{w}_{\text {opt }}\left(t \mid T_{d_{S R}}\right) \mathbf{h}_{S R^{*}}(t)\right|^{2}+N_{0}\right)}$. After further math314 ematical manipulations, the mutual information (MI) between $315 S$ and $D$, as well as the eavesdropper, can be written as

$$
\begin{aligned}
& I_{D}^{\mathrm{TBRS}}=\frac{1}{2} \log \left(1+\gamma_{D}^{\mathrm{TBRS}}\right)=\frac{1}{2} \log \left(1+\frac{\gamma_{S R} \gamma_{R^{*} D}}{\gamma_{S R}+\gamma_{R^{*} D}+1}\right) \\
& I_{E}^{\mathrm{TBRS}}=\frac{1}{2} \log \left(1+\gamma_{E}^{\mathrm{TBRS}}\right)=\frac{1}{2} \log \left(1+\frac{\gamma_{S R} \gamma_{R^{*} E}}{\gamma_{S R}+\gamma_{R^{*} E}+1}\right) .
\end{aligned}
$$

316 2) Joint Relay and Jammer Selection: Similarly, consider317 ing the unavailability of the instantaneous CSI regarding the 318 eavesdropper, we adopt a suboptimal RN/JN selection metric 319 conditioned on the outdated CSI as

$$
\begin{aligned}
& R^{*}=\arg \max _{R_{k} \in \mathcal{R}}\left\{\frac{\tilde{\gamma}_{R_{k} D}}{\mathbb{E}\left[\gamma_{R_{k} E}\right]}\right\} \\
& J^{*}=\arg \min _{R_{k} \in \mathcal{R}-R^{*}}\left\{\frac{\tilde{\gamma}_{R_{k} D}}{\mathbb{E}\left[\gamma_{R_{k} E}\right]}\right\}
\end{aligned}
$$

320 where $J^{*}$ is selected for minimizing the interference imposed 321 on $D$.

322 It should be noted that, to have the same transmit power as 323 that of the TBRS case, we assume that $P_{R *}+P_{J^{*}}=P_{R}$ for 324 our JRJS strategy and introduce $\lambda=P_{R^{*}} /\left(P_{R^{*}}+P_{J^{*}}\right)$ as the ratio of the relay's transmit power to the total power required 325 by the active relay and jammer.

In the cooperative phase, $R^{*}$ will also amplify the received 327 signal $y_{R^{*}}(t)$ by $G$ and forward it to $D$. At the same time, the 328 jammer $J^{*}$ will generate intentional interference to confuse $E, 329$ which will also cause interference at $D$. Consequently, the MI 330 between the terminals is given by

$$
I_{D}^{\mathrm{JRJS}}=\frac{1}{2} \log \left(1+\gamma_{D}^{\mathrm{JRJS}}\right)=\frac{1}{2} \log \left(1+\frac{\gamma_{S R} \frac{\gamma_{R^{*} D}}{\gamma_{J^{*} D}+1}}{\gamma_{S R}+\frac{\gamma_{R^{*} D}}{\gamma_{J^{*} D}+1}+1}\right)
$$

$$
I_{E}^{\mathrm{JRJS}}=\frac{1}{2} \log \left(1+\gamma_{E}^{\mathrm{JRJS}}\right)=\frac{1}{2} \log \left(1+\frac{\gamma_{S R} \frac{\gamma_{R E}}{\gamma_{J E}+1}}{\gamma_{S R}+\frac{\gamma_{R E}}{\gamma_{J E}+1}+1}\right)
$$

Remark 1: Generally, the optimal RN/JN selection scheme 332 should take into account the global SNR knowledge set 333 $\left\{\gamma_{S R}, \gamma_{R D}, \gamma_{R E}\right\}$. However, given the potentially excessive 334 implementational complexity overhead of the optimal selection 335 schemes and the unavailability of the global CSI, we employ 336 suboptimal selection schemes as in [12]. ${ }^{2}$ Furthermore, it is 337 commonly assumed that the average SNR of the eavesdropper 338 is available at the transmitter, which seems, somehow, not 339 reasonable. However, as stated in most of the literature, such as 340 [12]-[22], [24]-[28], and [30], provided that the eavesdropper 341 belongs to the network, which is also the case in our paper, 342 the related assumption might still be deemed reasonably. Addi- 343 tionally, as in [8], [11], [12], and [24], for mathematical conve- 344 nience, we assume that the relaying channels are independent 345 and identically distributed and that we have $\mathbb{E}\left[\gamma_{S R_{k}}\right]=\bar{\gamma}_{S R}, 346$ $\mathbb{E}\left[\gamma_{R_{k} D}\right]=\bar{\gamma}_{R D}$, and $\mathbb{E}\left[\gamma_{R_{k} E}\right]=\bar{\gamma}_{R E}$. The distances between 347 the relays are assumed to be much smaller than the distances 348 between relays and source/destination/eavesdropper; hence, the 349 corresponding path losses among the different relays are ap- 350 proximately the same. This assumption is reasonable both for 351 WSNs and for MANETs associated with a symmetric clustered 352 relay configuration, and it may be also satisfied as valid by 353 classic cellular systems in a statistical sense [11].

\section{SECURE Transmission Without JAMMing}

355

Here, we endeavor to characterize both the reliability and 356 security performance comprehensively of the TBRS scheme. 357 We first derive closed-form expressions for both the COP and 358 the SOP. Then, the RSR is introduced through the asymptotic 359 analysis of the COP and the SOP. Furthermore, we propose 360 the novel definition of the RSCP and the effective secrecy 361 throughput.

\footnotetext{
${ }^{2}$ To further alleviate the cooperation-related overhead, the selection criterion is based on the $R \rightarrow D$ link, since the second hop plays a dominant role in determining the received SNR, because the first hop corresponds to a multipleinput-single-output channel with the aid of multiple antennas, and hence, it is more likely to be better than the second hop. The optimal selection based on both hops is beyond the scope of this work.
} 


\section{A. COP and SOP}

364 When the perfect instantaneous CSI of the eavesdropper's 365 channel and even the legitimate users' channel is unavailable, 366 alternative definitions of the outage probability may be adopted 367 for the statistical characterization of the attainable secrecy 368 performance, particularly for delay-limited applications. Based 369 on [31, Def. 2], perfect secrecy cannot be achieved, when we 370 have $R_{e}<I_{E}$, where $I_{E}$ denotes the MI between the source 371 and the eavesdropper. Encountering this event is termed as a 372 secrecy outage. Furthermore, the destination is unable to flaw373 lessly decode the received codewords when $R_{0}>I_{D}$, which is 374 termed as a connection outage. The grade of reliability and the 375 grade of security maintained by a transmission scheme may be 376 then quantified by the COP and the SOP, respectively.

377 We continue by presenting our preliminary results versus the 378 point-to-point SNRs. Let us denote the cumulative distribution 379 function (CDF) and the probability density function (PDF) of a 380 random variable $X$ by $F_{X}(x)$ and $f_{X}(x)$, respectively. On one 381 hand, the PDF of $\gamma_{S R}$ using [29, eq. (15)] is given by

$$
\begin{array}{r}
f_{\gamma_{S R}}(x)=\sum_{n=0}^{N_{t}-1}\left(\begin{array}{c}
N_{t}-1 \\
n
\end{array}\right) \frac{\rho_{S R}^{2\left(N_{t}-1-n\right)}\left(\bar{\gamma}_{S R}\left(1-\rho_{S R}^{2}\right)\right)^{n}}{\bar{\gamma}_{S R}^{N_{t}}\left(N_{t}-1-n\right) !} \\
\times x^{N_{t}-1-n} e^{\frac{-x}{\gamma_{S R}}}
\end{array}
$$

382 whereas its CDF is given by

$$
\begin{aligned}
F_{\gamma_{S R}}(x)=1-\sum_{n=0}^{N_{t}-1} & \sum_{m=0}^{N_{t}-1-n}\left(\begin{array}{c}
N_{t}-1 \\
n
\end{array}\right) \\
& \times \frac{\rho_{S R}^{2\left(N_{t}-1-n\right)}\left(1-\rho_{S R}^{2}\right)^{n}}{m ! \bar{\gamma}_{S R}^{m}} x^{m} e^{\frac{-x}{\gamma_{S R}}} .
\end{aligned}
$$

383 On the other hand, for the instantaneous SNR of the $R \rightarrow$ $384 D$ hop, according to the principles of concomitants or induced 385 order statistics, the CDF of $\gamma_{R^{*} D}$ can be derived as in [32]

$$
F_{\gamma_{R^{*} D}}(y)=K_{r} \sum_{k=0}^{K_{r}-1}(-1)^{k}\left(\begin{array}{c}
K_{r}-1 \\
k
\end{array}\right) \frac{1-e^{\frac{-(k+1) y}{\left(k\left(1-\rho_{R D}^{2}\right)+1\right) \bar{\gamma}_{R D}}}}{k+1}
$$

386

Thus, the COP of the TBRS strategy is given by

$$
P_{\mathrm{co}}^{\mathrm{TBRS}}\left(R_{0}\right)=\operatorname{Pr}\left[I_{D}^{\mathrm{TBRS}}<R_{0}\right]=F_{\gamma_{D}^{\mathrm{TBRS}}}\left(\gamma_{t h}^{D}\right)
$$

387 where we have $\gamma_{t h}^{D}=2^{2 R_{0}}-1$, and the CDF of $\gamma_{D}^{\text {TBRS }}$ can be 388 calculated as

$$
\begin{aligned}
& F_{\gamma_{D}^{\mathrm{TBRS}}}(x)=1 \\
& \quad-\int_{0}^{\infty}\left[1-F_{\gamma_{R^{*} D}}\left(\frac{x z+x(x+1)}{z}\right)\right] f_{\gamma_{S R^{*}}}(z+x) d z .
\end{aligned}
$$

389 Consequently, by substituting (8) and (10) into (12) and using 390 [33, eq. (3.471.9)], we arrive at a closed-form expression for

$$
F_{\gamma_{D}^{\mathrm{TBRS}}}(x) \text { as }
$$

$$
\begin{aligned}
F_{\gamma_{D}^{\mathrm{TBRS}}}(x)= & 1-2 \sum_{n=0}^{N_{t}-1} \sum_{k=0}^{K_{r}-1} \sum_{m=0}^{N_{t}-1-n}(-1)^{k} K_{r}\left(\begin{array}{c}
N_{t}-1 \\
n
\end{array}\right) \\
& \times\left(\begin{array}{c}
K_{r}-1 \\
k
\end{array}\right)\left(\begin{array}{c}
N_{t}-1-n \\
m
\end{array}\right) \\
& \times \frac{\rho_{S R}^{2\left(N_{t}-1-n\right)}\left(1-\rho_{S R}^{2}\right)^{n} x^{N_{t}-1-n-m}}{\left(N_{t}-1-n\right) !(k+1) \bar{\gamma}_{S R}^{N_{t}-n}} \\
& \times\left[\frac{\bar{\gamma}_{S R} x(x+1)}{\omega_{k} \bar{\gamma}_{R D}}\right]^{\frac{m+1}{2}} \\
& \times e^{-\left(\frac{\bar{\gamma}_{S R}+\omega_{k} \bar{\gamma}_{R D}}{\omega_{k} \bar{\gamma}_{S R} \bar{\gamma}_{R D}}\right) x} K_{m+1}\left(2 \sqrt{\frac{x(x+1)}{\omega_{k} \bar{\gamma}_{S R} \bar{\gamma}_{R D}}}\right)
\end{aligned}
$$

where we have $\omega_{k}=\left(k\left(1-\rho_{R D}^{2}\right)+1\right) /(k+1)$. Then, by 392 substituting $x=\gamma_{t h}^{D}$ into (13), we obtain $P_{\text {co }}^{\text {TBRS }}$. 393

Furthermore, the SOP of the TBRS strategy may be expressed as 394

$$
P_{\mathrm{so}}^{\mathrm{TBRS}}\left(R_{0}, R_{s}\right)=\operatorname{Pr}\left[I_{E}^{\mathrm{TBRS}}>R_{0}-R_{s}\right]=1-F_{\gamma_{E}^{\mathrm{TBRS}}}\left(\gamma_{t h}^{E}\right)
$$

where we have $\gamma_{t h}^{E}=2^{2\left(R_{0}-R_{s}\right)}-1$. Similarly, we may calcu- 395 late the CDF of $\gamma_{E}^{\mathrm{TBRS}}$ in (14) as

$$
\begin{aligned}
F_{\gamma_{E}^{\mathrm{TBRS}}}(x)= & 1-2 \sum_{n=0}^{N_{t}-1} \sum_{m=0}^{N_{t}-1-n}\left(\begin{array}{c}
N_{t}-1 \\
n
\end{array}\right)\left(\begin{array}{c}
N_{t}-1-n \\
m
\end{array}\right) \\
& \times \frac{\rho_{S R}^{2\left(N_{t}-1-n\right)}\left(1-\rho_{S R}^{2}\right)^{n} x^{N_{t}-1-n-m}}{\left(N_{t}-1-n\right) ! \bar{\gamma}_{S R}^{N_{t}-n}} \\
& \times\left[\frac{\bar{\gamma}_{S R} x(x+1)}{\bar{\gamma}_{R E}}\right]^{\frac{m+1}{2}} \\
& \times e^{-\left(\frac{\bar{\gamma}_{S R}+\bar{\gamma}_{R E}}{\bar{\gamma}_{S R} \bar{\gamma}_{R E}}\right) x} K_{m+1}\left(2 \sqrt{\frac{x(x+1)}{\bar{\gamma}_{S R} \bar{\gamma}_{R E}}}\right) .
\end{aligned}
$$

Then, by substituting $x=\gamma_{t h}^{E}$ into (15), we can derive $P_{\text {so }}^{\text {TBRS }} .397$

The COP and the SOP in (11) and (14) characterize the at- 398 tainable reliability and security performance, respectively, and 399 can be regarded as the detailed requirements of accurate system 400 design. From the definition of COP and SOP, it is clear that 401 the reliability of the main link can be improved by increasing 402 the transmit SNR (or decreasing its data rate) to reduce the 403 COP, which unfortunately increases the risk of eavesdropping. 404 Thus, a tradeoff between reliability and security may be struck, 405 despite the fact that closed-from expressions cannot be obtained 406 as in [11]. Furthermore, we denote the minimal reliability and 407 security requirements by $v$ and $\delta$, where the feasible range of 408 the reliability constraint is $0<v<1$. Bearing in mind that 409 the COP is a monotonously increasing function of $R_{0}$, the 410 corresponding threshold of the codeword transmission rate is 411 $R_{0}^{t h}=\arg \left\{P_{\mathrm{co}}^{\mathrm{TBRS}}\left(R_{0}\right)=v\right\}$, which leads to a lower bound of 412 the SOP, when we have $\left(R_{0}-R_{s}\right) \rightarrow R_{0}^{t h}$. Thus, the feasible 413 range of $\delta$ is $P_{\text {so }}^{\text {TBRS }}\left(R_{0}^{t h}, 0\right)<\delta<1$. The preceding analysis 414 indicates that, given a reliability constraint $v$, the lower bound 415 of the security constraint is determined. 


\section{B. Reliability-Security Ratio}

418 Here, we will focus our attention on the asymptotic analysis 419 of the COP and the SOP in the high-SNR regime. Then, inspired 420 by [25], we introduce the concept of the RSR for characterizing 421 the direct relationship between reliability and security.

422 Proposition 1: Based on the asymptotic probabilities of $P_{\mathrm{co}}$ 423 and $P_{\text {so }}$ at high SNRs, ${ }^{3}$ the RSR is defined as

$$
P_{\mathrm{co}}\left(R_{0}\right)=\Lambda\left[1-P_{\mathrm{so}}\left(R_{0}, R_{s}\right)\right]
$$

424 where $\Lambda=\lim _{\eta \rightarrow \infty} P_{\mathrm{co}} /\left(1-P_{\mathrm{so}}\right)$, which represents the im425 provement in COP upon decreasing the SOP. More specifically, 426 since the reduction of the SOP/COP must be followed by an 427 improvement of COP/SOP, a lower $\Lambda$ implies that, when the 428 security is reduced, the reliability is improved, and vice versa. 429 Thus, for the TBRS scheme studied earlier, the RSR is derived 430 as (17), shown at the bottom of the page.

431 Proof: The proof is given in Appendix B.

432 Remark 2: It can be seen from the preceding expression 433 that the factor $\Lambda$ is independent of the transmit SNR, but 434 directly depends on the channel gains, the rate pair $\left(R_{0}, R_{s}\right)$, 435 and the number of TAs and relays. For a given $R_{s}$, reducing $436 R_{0}$ to enhance the reliability may erode the security, because $437\left(R_{0}-R_{s}\right)$ is also reduced. Conversely, increasing $R_{0}$ provides 438 more redundancy for protecting the security of the information, 439 but simultaneously, the reliability is reduced. Hence, the RSR 440 analysis underlines an important point of view concerning how 441 to balance the reliability versus security tradeoff by adjusting $442\left(R_{0}, R_{s}\right)$. Furthermore, as long as a CSI feedback delay exists, 443 the RSR has an intimate relationship with $\rho_{S R}$ and $\rho_{R D}$. It is 444 clear that the value of $\Lambda^{\text {TBRS }}$ decreases as $\rho_{R D}$ increases, which 445 is due to the fact that the relay selection process only improves 446 the reliability of the legitimate user. On the other hand, since 447 we always have the conclusion that $\sum_{k=0}^{K_{r}-1}(-1)^{k}\left(\begin{array}{c}K_{r}-1 \\ k\end{array}\right)\left(K_{r} /\right.$ $\left.448\left(k\left(1-\rho_{R D}^{2}\right)+1\right)\right)<1$, when $\sigma_{R D}^{2}$ and $\sigma_{R E}^{2}$ are comparable, $449 \Lambda^{\text {TBRS }}$ will be reduced as $\rho_{S R}$ increases. This observation 450 implies that, although both $P_{\mathrm{co}}$ and $\left(1-P_{\mathrm{so}}\right)$ are reduced 451 when the first-hop CSI becomes better, the improvement of

\footnotetext{
${ }^{3}$ Assume equal power allocation between $S$ and the relay, yielding $P_{S}=$ $P_{R}=P$, and define $\eta=P / N_{0}$ as the transmit SNR [24].
}

the reliability is more substantial than the security loss, as $\rho_{S R} 452$ increases.

\section{Effective Secrecy Throughput}

It should be noted that the COP and SOP metrics ignore the 455 correlation between these two outage events. More specifically, 456 in contrast to the point-to-point transmission case, since the 457 $S \rightarrow R$ link's SNR included in the MI expressions of (3) and 458 (4), the secrecy outage and the connection outage are definitely 459 not independent of each other. Therefore, it might be of limited 460 benefit in evaluating the reliability or the security separately. 461 We note furthermore that, although another metric referred to 462 as the secrecy throughput was introduced as the product of the 463 successful decoding probability and of the secrecy rate [21], 464 [22], this definition ignores the fact that a reliable transmission 465 may be insecure, and the SOP is not taken into consideration. 466 Hence, this metric is unable to holistically characterize the 467 efficiency of our scheme, while capable of achieving both re- 468 liable and secure transmission. Therefore, here, we redefine the 469 effective secrecy throughput as the probability of a successful 470 transmission (reliable and secure) multiplied by the secrecy 471 rate, namely, as $\varsigma=R_{s} P_{R \& S}$, where the RSCP is defined as 472

$$
P_{R \& S}=\operatorname{Pr}\left\{I_{D}>R_{0}, I_{E}<R_{0}-R_{s}\right\}
$$

Upon substituting the expressions of $I_{D}$ and $I_{E}$ in (3) and (4) 473 into (18), we can rewrite $P_{R \& S}$ for the TBRS strategy in (19), 474 shown at the bottom of the page.

Finally, using the corresponding CDFs and PDFs of (8)-(10) 476 from our previous analysis, we can obtain $P_{R \& S}^{\mathrm{TBRS}}$ in (20), 477 shown at the bottom of the next page, as well as the secrecy 478 throughput.

479

Furthermore, considering the asymptotic result for RSCP at 480 high SNRs in (20) by applying the approximation $K_{v}(x) \approx 481$ $(v-1) ! / 2(x / 2)^{v}$ and closing the highest terms of $\eta$ after 482 invoking the McLaurin series representation for the exponential 483 function, the asymptotic effective secrecy throughput can be 484 approximated as

485

Remark 3: Given the definition of COP, SOP, and the secrecy 486 throughput result of (21), shown at the bottom of the next page, 487 it can be shown that, for a fixed $R_{s}$, if $R_{0}$ is too small, although 488

$$
\Lambda^{\mathrm{TBRS}}=\frac{\left[\left(1-\rho_{S R}^{2}\right)^{N_{t}-1}+\sum_{k=0}^{K_{r}-1}(-1)^{k}\left(\begin{array}{c}
K_{r}-1 \\
k
\end{array}\right) \frac{K_{r} \sigma_{S R}^{2}}{\left[k\left(1-\rho_{R D}^{2}\right)+1\right] \sigma_{R D}^{2}}\right]\left(2^{2 R_{0}}-1\right)}{\left[N_{t}\left(1-\rho_{S R}^{2}\right)^{N_{t}-1}+\sigma_{S R}^{2} / \sigma_{R E}^{2}\right]\left(2^{2\left(R_{0}-R_{s}\right)}-1\right)}
$$

$$
\begin{aligned}
P_{R \& S}^{\mathrm{TBRS}} & =\operatorname{Pr}\left\{\left\{\gamma_{S R}>\gamma_{t h}^{D}, \gamma_{R^{*} D}>\frac{\gamma_{t h}^{D} \gamma_{S R}+\gamma_{S R}}{\gamma_{S R}-\gamma_{t h}^{D}}\right\} \cap\left[\left\{\gamma_{S R}>\gamma_{t h}^{E}, \gamma_{R^{*} E}<\frac{\gamma_{t h}^{E} \gamma_{S R}+\gamma_{S R}}{\gamma_{S R}-\gamma_{t h}^{E}}\right\} \cup\left\{\gamma_{S R}<\gamma_{t h}^{E}\right\}\right]\right\} \\
& =\operatorname{Pr}\left\{\gamma_{S R}>\gamma_{t h}^{D}, \gamma_{R^{*} D}>\gamma_{t h}^{D}+\frac{\gamma_{t h}^{D}\left(\gamma_{t h}^{D}+1\right)}{\gamma_{S R}-\gamma_{t h}^{D}}, \gamma_{R^{*} E}<\gamma_{t h}^{E}+\frac{\gamma_{t h}^{E}\left(\gamma_{t h}^{E}+1\right)}{\gamma_{S R}-\gamma_{t h}^{E}}\right\}
\end{aligned}
$$


$489 P_{R S}$ may be high (i.e., close to 1 ), the value of $\varsigma$ remains small. 490 By contrast, if $R_{0}$ is too large, the value of $P_{\text {co }}$ is close to 1 , 491 and therefore, $\varsigma$ will also become small. This observation is 492 also suitable for $R_{s}$. Thus, as pointed out in the RSR analysis, 493 it is elusive to improve both the reliability and the security 494 simultaneously, but both of them are equally crucial in terms 495 of the effective secrecy throughput, which depends on the rate 496 pair $\left(R_{0}, R_{s}\right)$.

497 Additionally, (21) also reveals that increasing the SNR would 498 drastically reduce the effective secrecy throughput. For high 499 transmit SNRs, a high reliability can indeed be perfectly guar500 anteed, but at the same time, the grade of the security is severely 501 degraded. However, the probability of a reliable and simultane502 ously secure transmission will tend toward zero. Hence, we may 503 conclude that there exists an optimal SNR, which achieves the 504 maximal secrecy throughput.

505 In conclusion, adopting the appropriate code rate pair and 506 transmit SNR is crucial for achieving the maximum effective 507 secrecy throughput, which can be formulated as

$$
\begin{aligned}
& \max _{R_{0}, R_{s}, \eta} \varsigma\left(R_{0}, R_{s}\right)=R_{s} P_{R \& S}^{\mathrm{TBRS}} \\
& \text { s.t. } P_{\text {co }} \leq v, P_{\text {so }} \leq \delta, 0<R_{s}<R_{0}
\end{aligned}
$$

508 where $v$ and $\delta$ denote the system's reliability and security 509 requirements. Unfortunately, it is quite a challenge to find 510 the closed-form optimal solution to this problem due to the 511 complexity of the expressions. Although suboptimal solutions 512 can be found numerically (with the aid of gradient-based search 513 techniques), the secrecy throughput optimization problem and 514 the corresponding complexity analysis and performance com515 parisons are beyond the scope of this work.

\section{6 \\ IV. Secure Transmission With Jamming}

517 Here, we consider the extension of the aforementioned relay 518 selection approaches to systems additionally invoking relay- aided jamming. JRJS is based on the outdated but perfectly 519 estimated CSI, and the details have been presented in Section II. 520 We would also like to investigate the security performance 521 from an outage-based perspective. The COP, SOP, RSCP, and 522 effective secrecy throughput will be included.

\section{A. COP and SOP}

It is plausible that the main differences between the JRJS and 525 TBRS schemes are determined by the instantaneous SNR of the 526 $R \rightarrow D$ hop, where, now, a jammer is included. Based on our 527 preliminary results detailed for the point-to-point SNRs in (8) 528 and (10), we now focus our attention on the statistical analysis 529 of the SNR, including $J^{*}$. As stated for the JRJS scheme in 530 Section II, $J^{*}$ corresponds to the lowest $\tilde{\gamma}_{R_{k} D}$ and is selected 531 from the set $\left\{\mathcal{R}-R^{*}\right\}$. Recalling that $R^{*}$ is the best relay 532 of the second hop, we have $\tilde{\gamma}_{J^{*} D}=\min _{R_{k} \in \mathcal{R}-R^{*}}\left\{\tilde{\gamma}_{R_{k} D}\right\} \triangleq 533$ $\min _{R_{k} \in \mathcal{R}}\left\{\tilde{\gamma}_{R_{k} D}\right\}$ for $K_{r}>1$. Using the induced order statis- 534 tics, the corresponding CDF of $\gamma_{R^{*} D}$ is presented in (10), 535 whereas the PDF of $\gamma_{J^{*} D}$ can be formulated as

$$
f_{\gamma_{J^{*} D}}(x)=\frac{K_{r} \exp \left(\frac{-K_{r} x}{\left[\left(K_{r}-1\right)\left(1-\rho_{R D}^{2}\right)+1\right] \bar{\gamma}_{J D}}\right)}{\left[\left(K_{r}-1\right)\left(1-\rho_{R D}^{2}\right)+1\right] \bar{\gamma}_{J D}}
$$

Although the relay and jammer selection processes are not 537 entirely disjoint, we may exploit the assumption that $\gamma_{R^{*} D}$ and 538 $\gamma_{J^{*} D}$ are independent of each other, which is valid when the 539 number of relays is sufficiently high, as justified in [24]. Let us 540 define the signal-to-interference-plus-noise ratio of the second 541 hop as $\xi_{D}=\gamma_{R^{*} D} /\left(\gamma_{J^{*} D}+1\right)$, using (10) and (23), whose 542 CDF can be formulated as

$$
F_{\xi_{D}}(x)=1-K_{r} \sum_{k=0}^{K_{r}-1}(-1)^{k}\left(\begin{array}{c}
K_{r}-1 \\
k
\end{array}\right) \frac{\varphi_{k} e^{\frac{-x}{\bar{\gamma}_{R D} \omega_{k}}}}{(k+1)\left(x+\varphi_{k}\right)}
$$

where we have $\varphi_{k}=\lambda K_{r} \omega_{k} /\left(\left[\left(K_{r}-1\right)\left(1-\rho_{R D}^{2}\right)+1\right](1-\lambda)\right) .544$

$$
\begin{aligned}
P_{R \& S}^{\mathrm{TBRS}}= & \int_{\gamma_{t h}^{D}}^{\infty}\left[1-F_{\gamma_{R^{*}}}\left(\gamma_{t h}^{D}+\frac{\gamma_{t h}^{D}\left(\gamma_{t h}^{D}+1\right)}{x-\gamma_{t h}^{D}}\right)\right] F_{\gamma_{R^{*}}}\left(\gamma_{t h}^{E}+\frac{\gamma_{t h}^{E}\left(\gamma_{t h}^{E}+1\right)}{x-\gamma_{t h}^{E}}\right) f_{\gamma_{S R^{*}}}(x) d x \\
\approx & 2 \sum_{n=0}^{N_{t}-1} \sum_{k=0}^{K_{r}-1} \sum_{m=0}^{N_{t}-1-n}(-1)^{k}\left(\begin{array}{c}
K_{r}-1 \\
k
\end{array}\right)\left(\begin{array}{c}
N_{t}-1 \\
n
\end{array}\right)\left(\begin{array}{c}
N_{t}-1-n \\
m
\end{array}\right) \frac{K_{r} \rho_{S R}^{2\left(N_{t}-1-n\right)}\left(1-\rho_{S R}^{2}\right)^{n}\left(\gamma_{t h}^{D}\right)^{N_{t}-1-n-m}}{\left(N_{t}-1-n\right) !(k+1) \bar{\gamma}_{S R}^{N_{t}-n-(m+1) / 2}} \\
& \times \exp \left[-\left(\frac{\gamma_{t h}^{D}}{\bar{\gamma}_{S R}}+\frac{\gamma_{t h}^{D}}{\omega_{k} \bar{\gamma}_{R D}}\right)\right]\left[\left(\frac{\gamma_{t h}^{D}\left(\gamma_{t h}^{D}+1\right)}{\omega_{k} \bar{\gamma}_{R D}}\right)^{\frac{m+1}{2}} K_{m+1}\left(2 \sqrt{\frac{\gamma_{t h}^{D}\left(\gamma_{t h}^{D}+1\right)}{\omega_{k} \bar{\gamma}_{S R} \bar{\gamma}_{R D}}}\right)\right. \\
& \left.-\exp \left(\frac{-\gamma_{t h}^{E}}{\bar{\gamma}_{R E}}\right)\left(\frac{\gamma_{t h}^{D}\left(\gamma_{t h}^{D}+1\right)}{\omega_{k} \bar{\gamma}_{R D}}+\frac{\gamma_{t h}^{E}\left(\gamma_{t h}^{E}+1\right)}{\bar{\gamma}_{R E}+\gamma_{t h}^{D}-\gamma_{t h}^{E}}\right)^{\frac{m+1}{2}} K_{m+1}\left(2 \sqrt{\frac{\gamma_{t h}^{D}\left(\gamma_{t h}^{D}+1\right)}{\omega_{k} \bar{\gamma}_{S R} \bar{\gamma}_{R D}}+\frac{\gamma_{t h}^{E}\left(\gamma_{t h}^{E}+1\right)}{\bar{\gamma}_{S R}\left(\bar{\gamma}_{R E}+\gamma_{t h}^{D}-\gamma_{t h}^{E}\right)}}\right)\right]
\end{aligned}
$$

$$
\tilde{\varsigma}^{\mathrm{TBRS}}\left(R_{0}, R_{s}, \eta\right)=R_{s}\left\{1-\left[\frac{N_{t}\left(1-\rho_{S R}^{2}\right)^{N_{t}-1}}{\sigma_{S R}^{2}}+\sum_{k=0}^{K_{r}-1} \frac{K_{r}(-1)^{k}}{\left[k\left(1-\rho_{R D}^{2}\right)+1\right] \sigma_{R D}^{2}}\left(\begin{array}{c}
K_{r}-1 \\
k
\end{array}\right)\right] \times \frac{2^{2 R_{0}}-1}{\eta}\right\} \frac{2^{2\left(R_{0}-R_{s}\right)}-1}{\sigma_{R E}^{2} \eta}
$$


545 As far as the eavesdropper is concerned, $\gamma_{R^{*} E}$ and $\gamma_{J^{*} E}$ 546 are independent and exponentially distributed. Furthermore, for $547 \xi_{E}=\gamma_{R^{*} E} /\left(\gamma_{J^{*} E}+1\right)$, we have

$$
F_{\xi_{E}}(x)=1-\frac{\phi}{x+\phi} e^{\frac{-x}{\bar{\gamma}_{R E}}}
$$

548 where $\phi=\lambda /(1-\lambda)$. According to the definition of COP and 549 SOP in Section III-A, we can obtain the following closed-form 550 approximations of the COP and the SOP. ${ }^{4}$

551 Lemma 1: The COP and the SOP of the JRJS strategy 552 associated with feedback delays are approximated by

$$
\begin{aligned}
P_{\mathrm{co}}^{\mathrm{JRJS}}\left(R_{0}\right) \approx & 1-\sum_{n=0}^{N_{t}-1} \sum_{k=0}^{K_{r}-1} \sum_{m=0}^{N_{t}-1-n}\left(\begin{array}{c}
N_{t}-1 \\
n
\end{array}\right) \\
& \times\left(\begin{array}{c}
K_{r}-1 \\
k
\end{array}\right)\left(\begin{array}{c}
N_{t}-1-n \\
m
\end{array}\right) \\
& \times \frac{(-1)^{k}\left(K_{r}+1\right) \rho_{S R}^{2\left(N_{t}-1-n\right)}\left(1-\rho_{S R}^{2}\right)^{n}}{\left(N_{t}-1-n\right) !(k+1) \bar{\gamma}_{S R}^{N_{t}-n}} \\
& \times \frac{\Gamma(m+2) \hat{\varphi}_{k}\left(\gamma_{t h}^{D}\right)^{N_{t}-n}\left(\gamma_{t h}^{D}+1\right)^{m+1}}{\left(\gamma_{t h}^{D}+\hat{\varphi}_{k}\right)^{m+2}} \\
& \times \exp \left[\begin{array}{c}
\gamma_{t h}^{D}\left(\hat{\varphi}_{k}-1\right) \\
\bar{\gamma}_{S R}\left(\gamma_{t h}^{D}+\hat{\varphi}_{k}\right)
\end{array}\right] \\
& \times \Gamma\left(-m-1, \frac{\gamma_{t h}^{D}\left(\gamma_{t h}^{D}+1\right)}{\bar{\gamma}_{S R}\left(\gamma_{t h}^{D}+\hat{\varphi}_{k}\right)}\right)
\end{aligned}
$$

553 where $\quad \hat{\varphi}_{k}=K_{r} \lambda \omega_{k} \eta \sigma_{R D}^{2} /\left(\left[\left(K_{r}-1\right)\left(1-\rho_{R D}^{2}\right)+1\right](1-\right.$ $\left.554 \lambda) \eta \sigma_{R D}^{2}+K_{r}\right)$, and

$$
\begin{aligned}
P_{\mathrm{so}}^{\mathrm{JRJS}}\left(R_{0}, R_{s}\right) \approx & \sum_{n=0}^{N_{t}-1} \sum_{m=0}^{N_{t}-1-n}\left(\begin{array}{c}
N_{t}-1 \\
n
\end{array}\right) \\
& \times \frac{\rho_{S R}^{2\left(N_{t}-1-n\right)}\left(1-\rho_{S R}^{2}\right)^{n}}{m ! \bar{\gamma}_{S R}^{m}} \\
& \times \frac{\left(2 \gamma_{t h}^{E}\right)^{m} \phi}{\left(2 \gamma_{t h}^{E}+\phi\right)} \exp \left[-\left(\frac{2 \gamma_{t h}^{E}}{\bar{\gamma}_{S R}}+\frac{2 \gamma_{t h}^{E}}{\bar{\gamma}_{R E}}\right)\right] .
\end{aligned}
$$

555 Proof: The proof is given in Appendix B.

556 The feasible range of the reliability constraint is similar to 557 that of the TBRS strategy, and hence, it is omitted here.

\section{B. Reliability-Security Ratio}

559 Lemma 2: Recalling the definition in Section III, the RSR 560 for the JRJS strategy may be expressed in (28), shown at the 561 bottom of the page.

${ }^{4}$ When we have $\lambda \rightarrow 1$, (24) will degenerate into the TBRS case seen in (10). The performance analysis of the JRJS will be presented separately in the following, since several approximations have to be included.
It can be seen from the previous expression that, in contrast 562 to the analysis of the TBRS strategy operating without jam- 563 ming, for a fixed SNR threshold, the CDF of the second-hop 564 SNR will converge to a nonzero limit. We also find that this 565 limit is determined by the power sharing ratio between the 566 relay and the jammer. Furthermore, according to the analy- 567 sis of the TBRS strategy, for $\eta \rightarrow \infty$, we have $F_{\gamma_{S R^{*}}}(x) \rightarrow 568$ 0 . Thus, by exploiting the tight upper bound that $\gamma_{D}^{\mathrm{TBRS}} \leq 569$ $\min \left\{\gamma_{S R}, \gamma_{R^{*} D}\right\}$ and $\gamma_{E}^{\mathrm{TBRS}} \leq \min \left\{\gamma_{S R}, \gamma_{R^{*} E}\right\}$, we have 570 $P_{\text {co }}^{\text {JRS }, \infty} \rightarrow F_{\gamma_{\xi_{D}}}\left(\gamma_{t h}^{D}\right)$ and $1-P_{\text {so }}^{\text {JRJS }, \infty} \rightarrow F_{\gamma_{\xi_{E}}}\left(\gamma_{t h}^{E}\right)$. Finally, 571 substituting the corresponding results into (16), we arrive at the 572 RSR of the JRJS strategy.

573

Remark 4: It can be seen from the RSR expression of (28) 574 again that the rate-pair setting $\left(R_{0}, R_{s}\right)$ has an inconsistent 575 influence on the RSR, and hence, we have to carefully adjust $R_{0} 576$ and $R_{s}$ to balance the reliability versus security performance. 577 Let us now focus our attention on the differences between the 578 JRJS scheme and the TBRS arrangement.

579

First, we may find that the power sharing ratio $\lambda$ between 580 the relay and the jammer plays a very important role. The 581 optimization of $\lambda$ will be investigated from an effective secrecy 582 throughput optimization point of view in the following. 583

Second, it is plausible that, in contrast to the behavior of the 584 TBRS strategy, $\Lambda^{\text {JRJS }}$ of (28) is only related to the delay of the 585 second hop, but it is still a monotonically decreasing function of 586 $\rho_{R D}$. This implies that the improvement of the channel quality 587 of the JRJS will achieve a more pronounced COP improvement 588 than the associated SOP improvement. Furthermore, recalling 589 that the RSR is considered in the high-SNR region, it has no 590 dependence on the first hop quality. This is due to the fact that 591 if the first-hop channel quality is sufficiently high for ensuring 592 a successful transmission, the asymptotic CDFs of $\xi_{D}$ and $\xi_{E} 593$ in (29) and (30) associated with $\eta \rightarrow \infty$ will converge to a 594 nonzero limit at high SNRs, which ultimately dominates the 595 COP and the SOP.

\section{Effective Secrecy Throughput}

Before proceeding to the effective secrecy throughput analy- 598 sis, we also have to investigate the RSCP.

599

Lemma 3: The RSCP of our JRJS strategy may be approxi- 600 mated as in (31), shown at the bottom of the next page, where 601 we have $\theta_{1, k}=\left(\gamma_{t h}^{D}\left(\gamma_{t h}^{D}+1\right)\right) /\left(\gamma_{t h}^{D}+\hat{\varphi}_{k}\right), \theta_{2}=\gamma_{t h}^{D}-\gamma_{t h}^{E}+602$ $\left(\gamma_{t h}^{E}\left(\gamma_{t h}^{E}+1\right) /\left(\gamma_{t h}^{E}+\hat{\phi}\right)\right)$, and $\hat{\phi}=\lambda \eta \sigma_{R E}^{2} /\left((1-\lambda) \eta \sigma_{R E}^{2}+1\right) .603$

Proof: The proof is given in Appendix C. 604

Apart from the rate pair $\left(R_{0}, R_{s}\right)$, the aforementioned $P_{R \& S}^{\mathrm{JRJS}} 605$ of (31) is also a function of the power sharing ratio $\lambda$ between 606 the selected relay and the jammer.

607

Given the complexity of the RSCP expression, it is quite 608 a challenge to find a closed-form result for maximizing the 609

$$
\Lambda^{\mathrm{JRJS}}=\frac{\left(2^{2 R_{0}}-1\right)}{\left(2^{2\left(R_{0}-R_{s}\right)}-1\right)} \sum_{k=0}^{K_{r}-1}\left(\begin{array}{c}
K_{r}-1 \\
k
\end{array}\right) \frac{(-1)^{k} K_{r}\left[\left(K_{r}-1\right)\left(1-\rho_{R D}^{2}\right)+1\right]\left[\left(\lambda^{-1}-1\right)\left(2^{2\left(R_{0}-R_{s}\right)}-1\right)+1\right]}{\left[\left(K_{r}-1\right)\left(1-\rho_{R D}^{2}\right)+1\right](k+1)\left(\lambda^{-1}-1\right)\left(2^{2 R_{0}}-1\right)+K_{r}\left[k\left(1-\rho_{R D}^{2}\right)+1\right]}
$$


610 effective secrecy throughput that $\max _{0<\lambda<1} \varsigma=R_{s} P_{R \& S}^{\mathrm{JRJS}}$. Al611 ternatively, we can focus on the asymptotic analysis in the high612 SNR region and try to find a general closed-form solution for $\lambda$. 613 Specifically, when we have $\eta \rightarrow \infty, P_{R \& S}^{\text {JRJS }}$ will be dominated 614 by the channel quality of the second hop; hence, we have

$$
\begin{aligned}
P_{R \& S}^{\mathrm{JRJS}, \infty}\left(R_{0}, R_{s}, \lambda\right) & \approx \operatorname{Pr}\left\{\xi_{D}>\gamma_{t h}^{D}, \xi_{E}<\gamma_{t h}^{E}\right\} \\
& =\left[1-F_{\xi_{D}}\left(\gamma_{t h}^{D}\right)\right] F_{\xi_{E}}\left(\gamma_{t h}^{E}\right)
\end{aligned}
$$

615 where the approximation is based on the fact that, in contrast to 616 both $F_{\xi_{D}}\left(\gamma_{t h}^{D}\right)$ and $F_{\xi_{E}}\left(\gamma_{t h}^{E}\right)$, which converge to a nonzero limit 617 regardless of $\eta$, the first hop's $F_{\gamma_{S R}}(x)$ will tend to zero, and 618 hence, it can be neglected. Substituting the asymptotic results 619 of (29) and (30) into (33), we can obtain $P_{R \& S}^{J R J S}, \infty$. In contrast to 620 the TBRS case operating without jamming, as the SNR tends to $621 \infty$, the RSCP will tend to a nonzero value and, upon increasing 622 the transmit SNR beyond a certain limit, will no longer increase 623 the effective secrecy throughput.

624 Then, based on (32), we arrive at the approximated optimal 625 value $\lambda_{\text {opt }}$, which is the solution of the following equation:

$$
\frac{\partial P_{R \& S}^{\mathrm{JRJS}, \infty}\left(R_{0}, R_{s}, \lambda\right)}{\partial \lambda}=0 .
$$

626 Then, by exploiting the approximation of $\left[k\left(1-\rho_{R D}^{2}\right)+1\right] /$ $627(k+1) \approx 1-\rho_{R D}^{2}$ in (29) for a large $\rho_{R D}$ (practically, the CSI 628 delay is small, and $\rho_{R D} \rightarrow 1$ ), we have

$$
\lambda_{\text {subopt }}=\frac{\sqrt{\left[\left(K_{r}-1\right)\left(1-\rho_{R D}^{2}\right)+1\right] \gamma_{t h}}}{\sqrt{\left[\left(K_{r}-1\right)\left(1-\rho_{R D}^{2}\right)+1\right] \gamma_{t h}}+\sqrt{K_{r}\left(1-\rho_{R D}^{2}\right)}}
$$

629 where $\gamma_{t h}=\left(2^{2 R_{0}}-1\right)\left(2^{2\left(R_{0}-R_{s}\right)}-1\right)$. It is clear that this 630 value is determined by the number of relays and $\left(R_{0}, R_{s}\right)$.

\section{1}

\section{NUMERICAL RESUltS}

632 Both our numerical and Monte Carlo simulation results are 633 presented here for verifying the theoretical PLS performance 634 analysis of the multiple-relay-aided network under CSI feed-

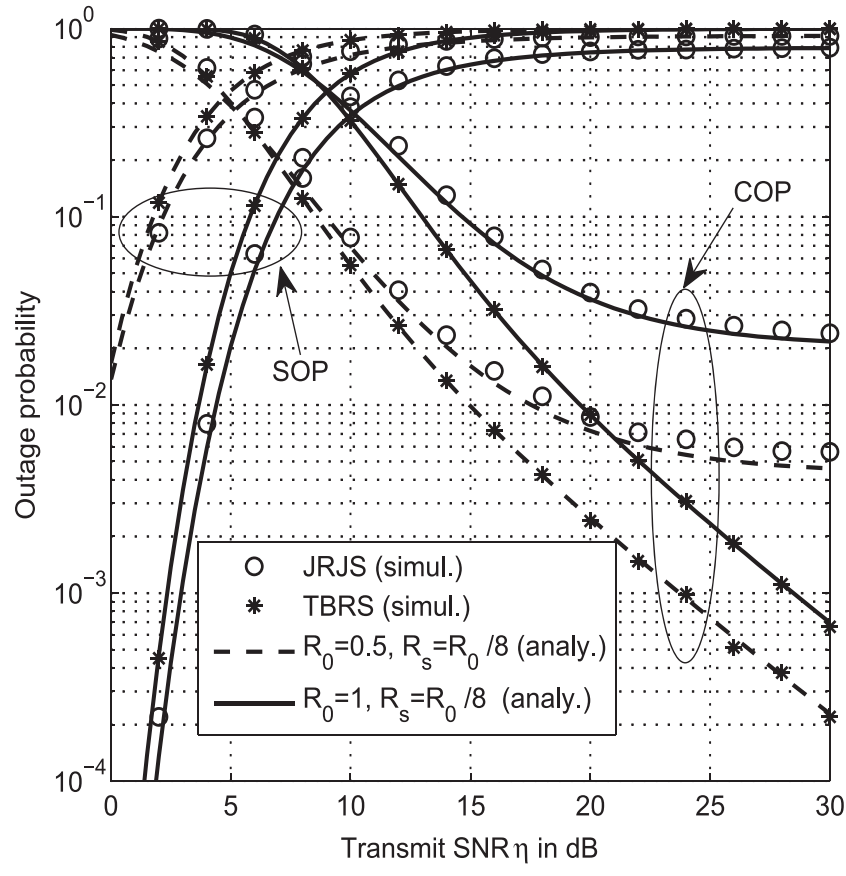

Fig. 2. COP and SOP versus transmit SNR for the TBRS and JRJS strategies in conjunction with different rate pairs, for $N_{t}=K_{r}=3, f_{d} T_{d}=0.1$, and $\lambda=1 / 10$.

back delays. Explicitly, the COP, SOP, RSCP, and RSR are 635 validated for both the TBRS and JRJS strategies. Furthermore, 636 the effects of feedback delays and system parameters (including 637 the transmission rate pair $\left(R_{0}, R_{s}\right)$ and the power sharing ratio 638 $\lambda$ between the relay and the jammer) on the achievable effective 639 secrecy throughput are evaluated. The Rayleigh fading model 640 is employed for characterizing all communication links in our 641 system. Additionally, we set the total power to $P=1$ and 642 $\sigma_{S R}^{2}=\sigma_{R D}^{2}=\sigma_{R E}^{2}=1$, and used $T_{d_{S R}}=T_{d_{R D}}=T_{d}$. 643

Fig. 2 plots the COP and the SOP versus the transmit SNR for 644 both the TBRS and JRJS strategies in conjunction with different 645 rate pairs. The analytical lines are plotted by using (11) and (14) 646 for the TBRS strategy and by using (26) and (27) for the JRJS 647

$$
\begin{aligned}
& P_{R \& S}^{\mathrm{JRJS}}\left(R_{0}, R_{s}, \lambda\right) \approx \sum_{n=0}^{N_{t}-1} \sum_{k=0}^{K_{r}-1} \sum_{m=0}^{N_{t}-1-n}(-1)^{k}\left(\begin{array}{c}
N_{t}-1 \\
n
\end{array}\right)\left(\begin{array}{c}
K_{r}-1 \\
k
\end{array}\right)\left(\begin{array}{c}
N_{t}-1-n \\
m
\end{array}\right) \\
& \times \frac{K_{r} \rho_{S R}^{2\left(N_{t}-1-n\right)}\left(1-\rho_{S R}^{2}\right)^{n} \hat{\varphi}_{k}\left(\gamma_{t h}^{D}\right)^{N_{t}-1-n-m}}{\left(N_{t}-1-n\right) !(k+1) \bar{\gamma}_{S R}^{N_{t}-n}\left(\gamma_{t h}^{D}+\hat{\varphi}_{k}\right) e^{\frac{\gamma_{t h}^{D}}{\bar{\gamma}_{S R}}+\frac{\gamma_{t h}^{D}}{\bar{\gamma}_{R D} \omega_{k}}}} \\
& \times\left\{\theta_{1, k}^{m+1} e^{\frac{\theta_{1}}{\bar{\gamma}_{S R}}} \Gamma(m+2) \Gamma\left(-m-1, \frac{\theta_{1, k}}{\bar{\gamma}_{S R}}\right)-\frac{\hat{\phi} e^{-\gamma_{t h}^{E} / \bar{\gamma}_{R E}}}{\left(\gamma_{t h}^{E}+\phi\right)\left(\theta_{1, k}-\theta_{2}\right)} \Gamma(m+3)\right. \\
& \times\left[\theta_{2}^{m+2} e^{\frac{\theta_{2}}{\bar{\gamma}_{S R}}} \Gamma\left(-m-2, \frac{\theta_{2}}{\bar{\gamma}_{S R}}\right)-\theta_{1, k}^{m+2} e^{\frac{\theta_{1}}{\bar{\gamma}_{S R}}} \Gamma\left(-m-2, \frac{\theta_{1, k}}{\bar{\gamma}_{S R}}\right)\right]+\Gamma(m+2)\left(\gamma_{t h}^{D}-\gamma_{t h}^{E}\right) \\
& \left.\times\left[\theta_{2}^{m+1} e^{\frac{\theta_{2}}{\bar{\gamma}_{S R}}} \Gamma\left(-m-1, \frac{\theta_{2}}{\bar{\gamma}_{S R}}\right)-\theta_{1, k}^{m+1} e^{\frac{\theta_{1}}{\bar{\gamma}_{S R}}} \Gamma\left(-m-1, \frac{\theta_{1, k}}{\bar{\gamma}_{S R}}\right)\right]\right\}
\end{aligned}
$$




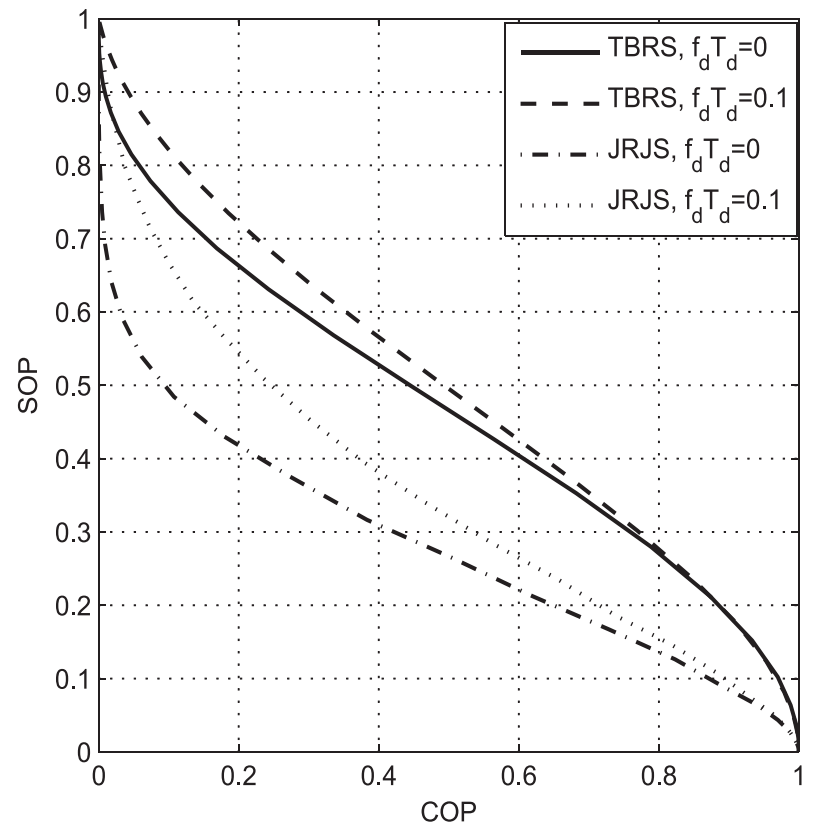

Fig. 3. SOP versus COP for the TBRS and JRJS strategies with different feedback delays for $N_{t}=K_{r}=3, R_{s}=R_{0} / 8$, and $\lambda=1 / 10$.

648 case, respectively. It can be clearly seen from the figure that the 649 analytical and simulated outage probability curves match well, 650 which confirms the accuracy of the mathematical analysis. As 651 expected, compared with the TBRS strategy, the SOP of the 652 JRJS strategy is much better, whereas the COP is worse. We 653 can also find that both the COP and the SOP will converge to an 654 outage floor at high SNRs for the JRJS strategy. The reason for 655 this is that the jammer also imposes interference on the destina656 tion and the interference inflicted increases with the SNR. Thus, 657 the designers have to take into account the tradeoff between 658 the reliability and the security and the interference imposed on $659 D$, particularly when considering the JRJS strategy. Moreover, 660 we can observe in Fig. 2 that increasing the transmission rate 661 decreases the COP and increases the SOP.

662 Fig. 3 further characterizes the SOP versus COP for both the 663 TBRS and JRJS strategies based on the numerical results in 664 Fig. 2, which shows the tradeoff between the reliability and the 665 security. It can be seen from the figure that the SOP decreases as 666 the COP increases, and for a specific COP, the SOP of the JRJS 667 scheme is strictly lower than that of TBRS. This confirms that 668 the JRJS scheme performs better than the conventional TBRS 669 scheme. Furthermore, the CSI feedback delay will also degrade 670 the system tradeoff performance.

671 Fig. 4 illustrates the RSCP versus transmit SNR for the 672 TBRS strategy in the context of different network configura673 tions, including different rate pairs, different number of relays, 674 and both perfect and outdated CSI feedback scenarios. The 675 analytical lines are plotted by using the approximation in (20). 676 We may conclude from the figure that the rate-pair setting $677\left(R_{0}, R_{s}\right)$ determines both the reliability and security transmis678 sion performance. These curves also show that the RSCP is a 679 concave function of the transmit SNR, whereas the continued 680 boosting of the SNR would only decrease the probability of 681 a successful transmission. We can observe from Fig. 4 that,

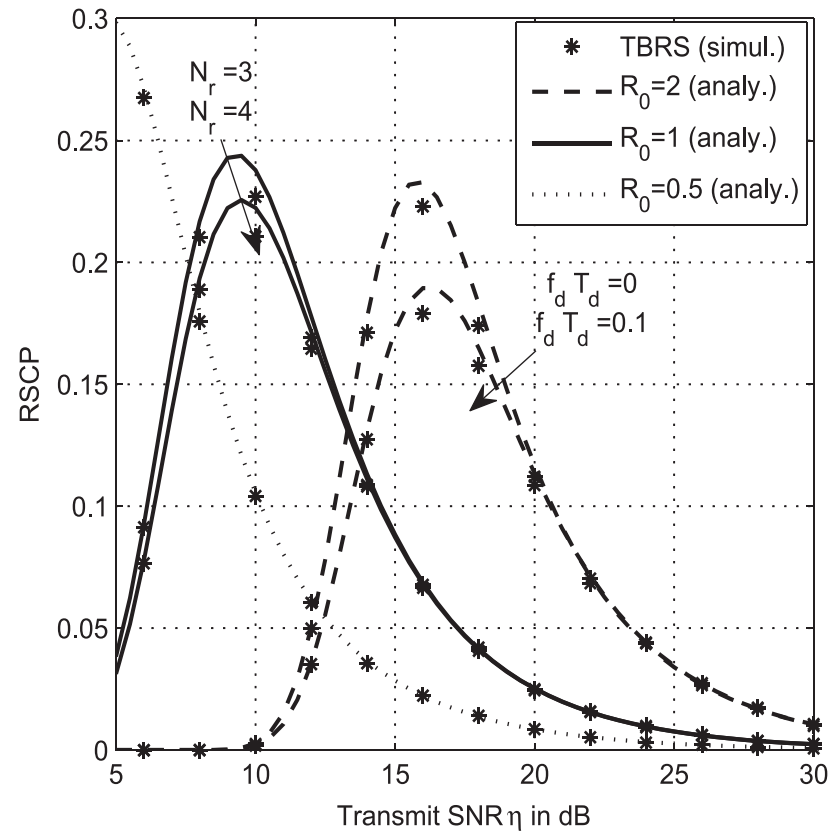

Fig. 4. RSCP versus transmit SNR for the TBRS strategy with different rate pairs for $N_{t}=K_{r}=3, f_{d} T_{d}=0.1$.

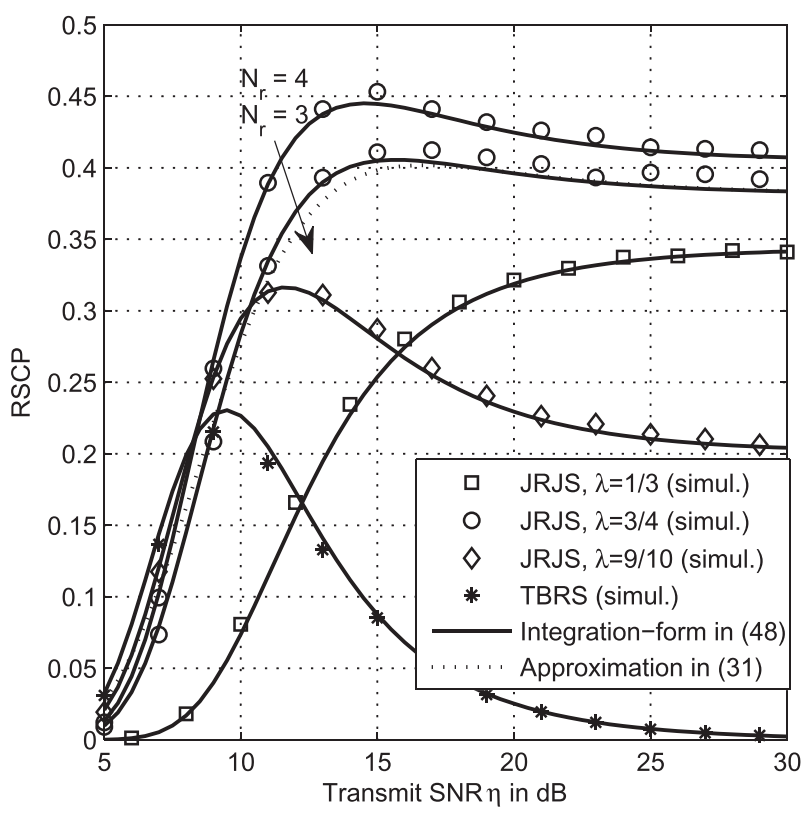

Fig. 5. RSCP versus transmit SNR for the JRJS strategy for different power sharing ratios $\lambda$ and for $N_{t}=K_{r}=3, f_{d} T_{d}=0.1$, and $R_{0}=1$, $R_{s}=R_{0} / 8$.

for a high transmit SNR, total reliability can be guaranteed, 682 whereas the associated grade of security is severely eroded. 683 Furthermore, increasing the number of relays and decreasing 684 the feedback delay will improve both the reliability and security 685 performance.

686

The RSCP of the JRJS strategy is presented in Fig. 5 for 687 different power sharing ratios between relaying and jamming. 688 Both the integration form (45) and the approximated closed 689 form in (31) match well with the Monte Carlo simulations. 690 The performance of the TBRS strategy is also included for 691 comparison. The JRJS scheme outperforms the TBRS operating 692 

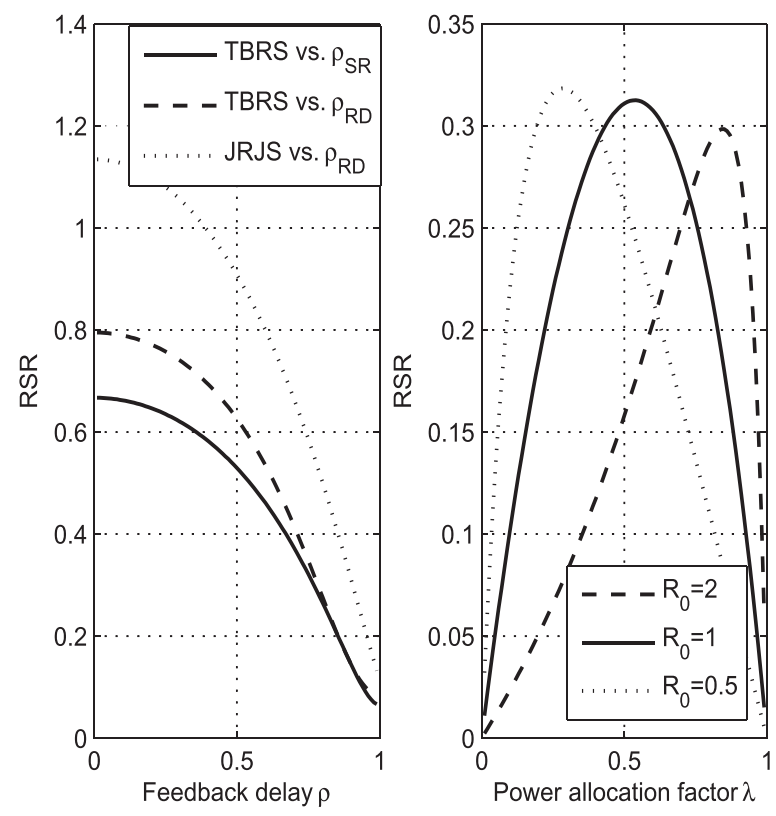

Fig. 6. RSR versus feedback delay coefficient $\left(R_{0}=1, R_{s}=R_{0} / 8, \lambda=\right.$ $3 / 4)$ and power sharing ratio $\lambda\left(R_{s}=R_{0} / 8, \rho_{S R}=\rho_{R D}=0.9\right)$ for the TBRS and JRJS strategies, with $N_{t}=K_{r}=3$.

693 without jamming under the scenario considered when encoun694 tering comparable relay-destination and relay-eavesdropper 695 channels. For some extreme configurations (when the relay696 eavesdropper links are comparatively weak), this statement 697 may not hold, but this scenario is beyond the scope of this 698 paper. The maximum RSCP appears at about $\eta=15 \mathrm{~dB}$ 699 for the JRJS strategy using $\lambda=3 / 4$, whereas it is $\eta=10 \mathrm{~dB}$ 700 for the TBRS strategy. Furthermore, as expected, increasing the 701 number of available relays and jamming nodes will always be 702 able to improve the reliability and security performance. How703 ever, the continued boosting of the jammer's power (decreasing $704 \lambda$ ) will not always improve the overall performance, because 705 the interference improves initially the security, but then, it starts 706 to reduce the reliability as $\lambda$ decreases. This further motivates 707 the designer to carefully take into account the power sharing 708 between relaying and jamming. The effect of the rate-pair 709 setting on the security and reliability of the JRJS strategy is 710 neglected here, which follows a similar trend to that of the 711 TBRS strategy.

712 Fig. 6 characterizes the RSR versus feedback delay and 713 power sharing ratio for both TBRS and JRJS, in which the 714 RSR curves are plotted by using (17) and (28), respectively. 715 The first illustration shows that the RSR decreases as the delay 716 coefficients $\left(\rho_{S R}\right.$ and $\left.\rho_{R D}\right)$, which confirms that the im717 provement of reliability becomes more pronounced than the 718 reduction of the security as the feedback delay decreases. 719 This observation implies an improvement in terms of the 720 security-reliability tradeoff. In addition, the RSR versus $\rho_{R D}$ 721 is larger than that of $\rho_{S R}$, which indicates that the impact of the 722 second-hop CSI feedback delay is more prominent. The other 723 illustration in the right demonstrates that the RSR is a concave 724 function of the power sharing ratio, which reflects the tradeoff 725 between the reliability and the security struck by adjusting $\lambda$.

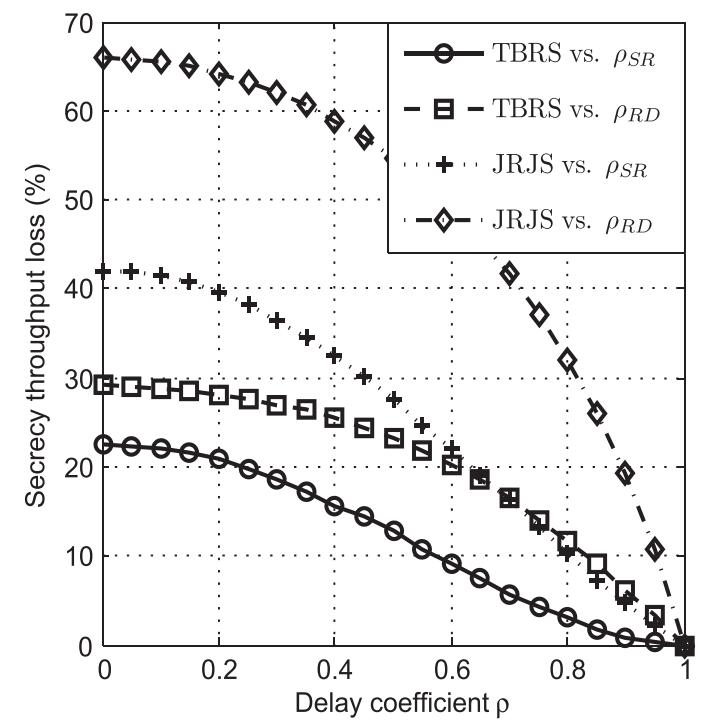

Fig. 7. Percentage secrecy throughput loss versus delay coefficients with $N_{t}=K_{r}=3, R_{0}=1, R_{s}=R_{0} / 8, \lambda=3 / 4$, and $\eta=10 \mathrm{~dB}$.

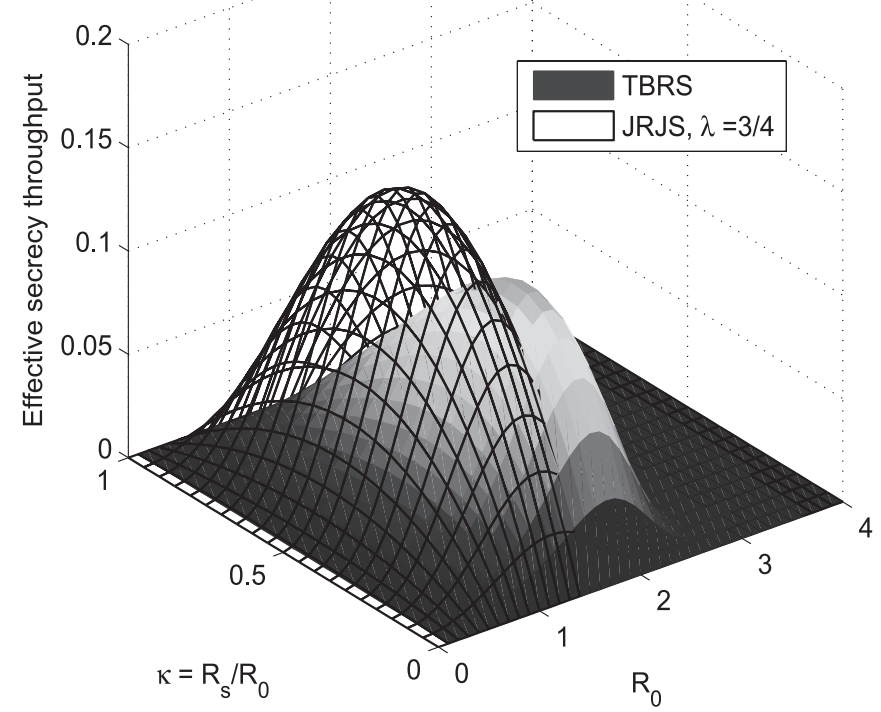

Fig. 8. Secrecy throughput versus $R_{0}$ and $\kappa=R_{s} / R_{0}$ for both the TBRS and JRJS strategies with $N_{t}=K_{r}=3, f_{d} T_{d}=0.1$, and $\eta=15 \mathrm{~dB}$.

To further evaluate the effect of feedback delays on the 726 secrecy performance, Fig. 7 plots the resultant percentage of 727 secrecy throughput loss versus the delay, which is defined as 728

$$
\varsigma_{\text {loss }}=\frac{\varsigma_{\text {no-delay }}-\varsigma_{\text {delay }}}{\varsigma_{\text {no-delay }}} \text {. }
$$

It can be seen from the figure that, compared with the TBRS 729 scheme, JRJS is more sensitive to the feedback delays. Further- 730 more, recalling that increasing the delay coefficient $\rho_{S R}$ of the 731 first hop improves the reliability, but at the same time also helps 732 the eavesdropper, it is not surprising that the secrecy throughput 733 loss due to the second-hop feedback delay is more pronounced. 734

Fig. 8 illustrates the achievable effective secrecy throughput 735 for both the TBRS and JRJS strategies versus the codeword 736 


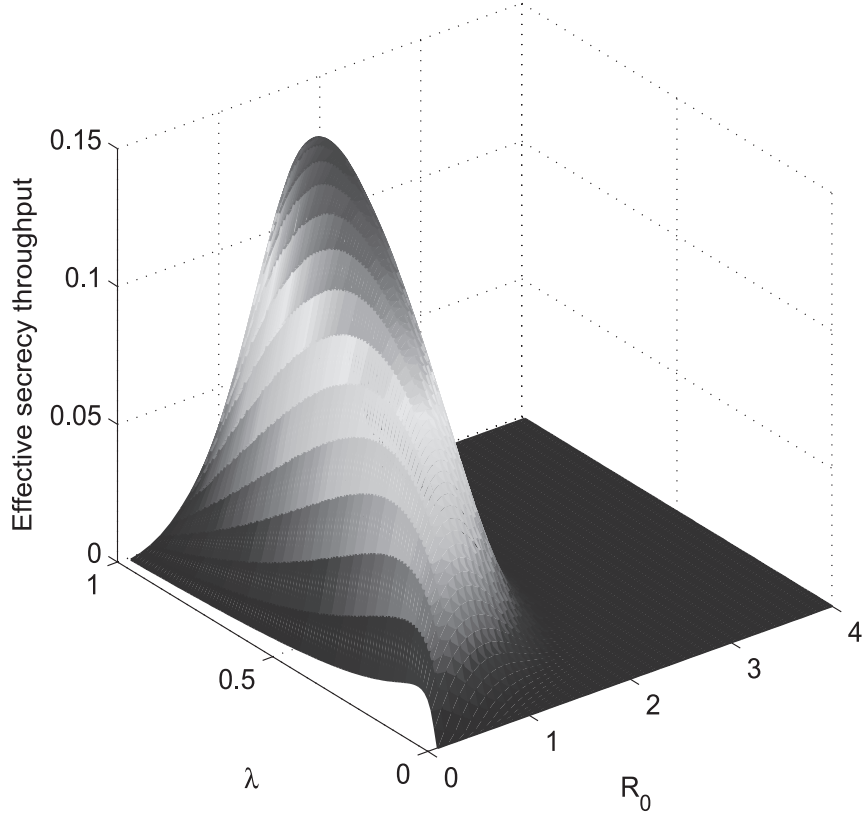

Fig. 9. Secrecy throughput versus $R_{0}$ and $\lambda$ for the JRJS strategy with $N_{t}=$ $K_{r}=3, f_{d} T_{d}=0.1, \eta=15 \mathrm{~dB}$, and $R_{s} / R_{0}=1 / 8$.

737 transmission rate $R_{0}$ and the secrecy code ratio $\kappa=R_{s} / R_{0}$ 738 with no outage constraints $(v=\delta=1)$. The values of the 739 effective secrecy throughput are plotted by using $\varsigma=R_{s} P_{R \& S}$. 740 We can observe in Fig. 8 that, subject to a fixed code rate 741 ratio $\kappa$, the effective secrecy throughput increases to a peak 742 value as $R_{0}$ reaches its optimal value and then decreases. This 743 phenomenon can be explained as follows. At a low transmission 744 rate, although the COP increases with $R_{0}$, which has a negative 745 effect on the effective secrecy throughput, both the secrecy 746 rate and the SOP performance will benefit. However, after 747 reaching the optimal $R_{0}$, the effective secrecy throughput drops 748 since the main link cannot afford a reliable transmission, and 749 the resultant COP increase becomes dominant. On the other 750 hand, subject to a fixed $R_{0}$ (which results in a constant COP), 751 the effective secrecy throughput is also a concave function 752 of $\kappa$, and increasing the code rate ratio ultimately results 753 in an increased secrecy information rate at the cost of an 754 increased SOP.

755 The achievable effective secrecy throughput for the JRJS 756 strategy is also presented in Fig. 8, and similar conclusions and 757 trends can be observed to that of the TBRS case. Additionally, 758 the comparison of the two strategies indicates that the JRJS 759 scheme attains a higher effective secrecy throughput than the 760 TBRS scheme operating without jamming, even if no power 761 sharing optimization has been employed.

762 Fig. 9 further illustrates the impact of power sharing between 763 the relay and the jammer on the achievable effective secrecy 764 throughput of the JRJS strategy versus $R_{0}$ in the absence of 765 outage constraints. Given a fixed code rate pair $\left(R_{0}, R_{s}\right)$, the 766 effective secrecy throughput follows the trend of the RSCP, 767 which is a concave function of $\lambda$, as shown in Fig. 6. The 768 interference introduced by the jammer initially improves both 769 the reliability and the security as $\lambda$ increases, but this trend is 770 reversed beyond a certain point.

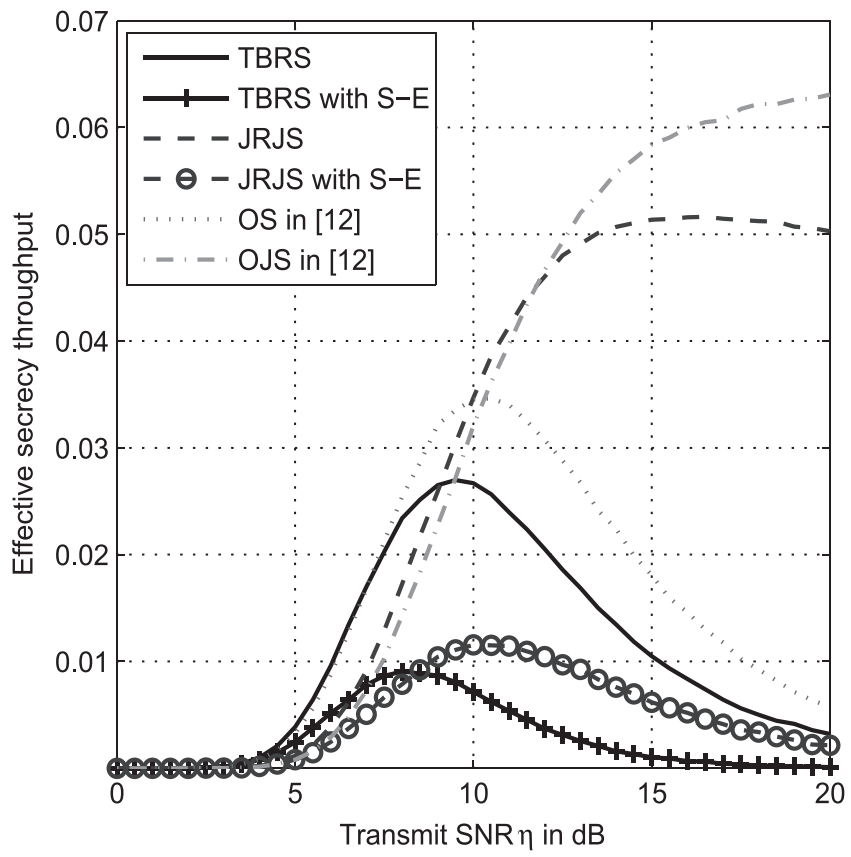

Fig. 10. Comparisons for different strategies with and without the S-E link, for $N_{t}=K_{r}=3, R_{0}=1, R_{s}=R_{0} / 8, f_{d} T_{d}=0.1$, and $\lambda=3 / 4$.

\section{DISCUSSION}

\section{A. Impact of the S-E Link}

We note that the introduction of the S-E link, i.e., the 773 information leakage in the first phase, is very critical to the 774 security. There are also some research studies focusing on 775 the corresponding secure transmission design and performance 776 evaluation for cooperative networks with the S-E link, such 777 as [15] and [16]. Here, we assume that the eavesdropper can 778 receive information directly from the source in the first phase. 779 Thus, following the steps in the prior sections, for the TBRS 780 and JRJS schemes, it is clear that the SNR experienced at the 781 eavesdropper should be rewritten as

$$
\tilde{\gamma}_{E}^{\tau}=\gamma_{S E}+\gamma_{E}^{\tau}
$$

where $\gamma_{S E}=P_{s}\left|\mathbf{w}_{\text {opt }}\left(t \mid T_{d_{S R}}\right) \mathbf{h}_{S E}(t)\right|^{2} / N_{0}$ follows the ex- 783 ponential distribution with the average value $\bar{\gamma}_{S E}, \tau=784$ \{TBRS, JRJS \}, and $\gamma_{E}^{\tau}$ has been defined in (4) and (7). $\quad 785$

Then, the corresponding SOP, RSCP, and effective secrecy 786 throughput have to be reconsidered. Unfortunately, to the best 787 of our knowledge, it is a mathematically intractable problem 788 to obtain closed-form results for the related performance eval- 789 uations. Therefore, we resorted to numerical simulations for 790 further investigating the impact of the S-E link. Fig. $10 \mathrm{com}-791$ pares the effective secrecy throughput of the TBRS and JRJS 792 schemes both with and without considering the direct S-E 793 link. It becomes clear that the information leakage in the first 794 phase will lead to a severe security performance degradation, 795 particularly for the JRJS scheme, which will no longer be 796 capable of maintaining a steady throughput at high SNRs. The 797 reason for this trend is that increasing the transmit SNR will 798 help the eavesdropper in the presence of the direct S-E link. 


\section{B. Comparisons}

801 Here, based on the outdated CSI assumption, we provide per802 formance comparisons with a range of other schemes advocated 803 in [12] with the aid of the proposed outage-based characteriza804 tion. Fig. 10 also incorporates our effective secrecy throughput 805 performance comparison, where the optimal selection (OS) 806 regime and the optimal selection combined with jamming (OSJ) 807 were proposed in [12]. They are formulated as

$$
\begin{gathered}
\text { OS }: R^{*}=\arg \max _{R_{k} \in \mathcal{R}}\left\{\frac{\tilde{\gamma}_{R_{k} D}}{\tilde{\gamma}_{R_{k} E}}\right\} \\
\text { OSJ : }\left\{\begin{array}{l}
R^{*}=\arg \max _{R_{k} \in \mathcal{R}}\left\{\frac{\tilde{\gamma}_{R_{k} D}}{\tilde{\gamma}_{R_{k} E}}\right\} \\
J^{*}=\arg \min _{R_{k} \in \mathcal{R}-R^{*}}\left\{\frac{\tilde{\gamma}_{R_{k} D}}{\tilde{\gamma}_{R_{k}} E}\right\}
\end{array}\right.
\end{gathered}
$$

808 where $\tilde{\gamma}_{R_{k} E}$ is the delayed version of the instantaneous CSI of 809 the R-E link. It should be noted that this constitutes an entirely 810 new performance characterization of these schemes from the 811 perspective of the effective secrecy throughput. It is shown in 812 Fig. 1 that the selection combined with jamming outperforms 813 the corresponding nonjamming techniques at high SNRs, albeit 814 this trend may no longer prevail at low SNRs. In comparison, 815 compared with those selections relying on the average SNRs of 816 the R-E link, the optimal selections relying on the idealized 817 simplifying assumptions of having global CSI (OS and OSJ 818 schemes) knowledge can only achieve throughput gains at high 819 SNRs due to the inevitable feedback delay.

\section{CONCLUSION}

821 An outage-based characterization of cooperative relay net822 works has been provided in the face of CSI feedback delays. 823 Two types of relaying strategies were considered, namely, the 824 TBRS strategy and the JRJS strategy. Closed-form expressions 825 of the COP, the SOP, and the RSCP, as well as of the RSR, 826 were derived. The RSR results demonstrated that the reliability 827 is improved more substantially than the security performance 828 when the CSI feedback delays are reduced. Furthermore, we 829 presented a modified effective secrecy throughput definition 830 and demonstrated that the JRJS strategy achieves a significant 831 effective secrecy throughput gain over the TBRS strategy. The 832 transmit SNR, the secrecy codeword rate setting, and the power 833 sharing ratio between the relay and jammer nodes play impor834 tant roles in striking a balance between the reliability and the 835 security in terms of the secrecy throughput. The impact of the 836 direct S-E link and the performance comparisons with other 837 selection schemes were also included. Additionally, our results 838 demonstrate that JRJS is more sensitive to the feedback delays 839 and that the secrecy throughput loss due to the second-hop 840 feedback delay is more pronounced than that due to the first841 hop one.
842

843
APPENDIX A

PROOF OF PROPOSITION 1
844 To simplify the asymptotic performance analysis, (3) can be 845 expressed in a more mathematically tractable form by the com846 monly used tight upper bound of $\gamma_{D}^{\mathrm{TBRS}} \leq \min \left\{\gamma_{S R}, \gamma_{R^{*} D}\right\}$ and $\gamma_{E}^{\mathrm{TBRS}} \leq \min \left\{\gamma_{S R}, \gamma_{R^{*} E}\right\}$. When we have $\eta \rightarrow \infty$, based 847 on the CDFs in (9) and (10) and closing the smallest order terms 848 of $x / \eta$, we have

$$
\begin{aligned}
F_{\gamma_{S R}}(x) \rightarrow 1- & {\left[\sum_{n=0}^{N_{t}-1}\left(\begin{array}{c}
N_{t}-1 \\
n
\end{array}\right) \rho_{S R}^{2\left(N_{t}-1-n\right)}\left(1-\rho_{S R}^{2}\right)^{n}\right.} \\
& +\sum_{n=0}^{N_{t}-2}\left(\begin{array}{c}
N_{t}-1 \\
n
\end{array}\right) \times \rho_{S R}^{2\left(N_{t}-1-n\right)} \\
& \left.\times\left(1-\rho_{S R}^{2}\right)^{n} \frac{x}{\bar{\gamma}_{S R}}+\mathcal{O}\left(\frac{x}{\bar{\gamma}_{S R}}\right)\right] \\
\times & {\left[1-\frac{x}{\bar{\gamma}_{S R}}+\mathcal{O}\left(\frac{x}{\bar{\gamma}_{S R}}\right)\right] } \\
=1- & {\left[1+\left(1-\left(1-\rho_{S R}^{2}\right)^{N_{t}-1}\right) \frac{x}{\bar{\gamma}_{S R}}+\mathcal{O}\left(\frac{x}{\bar{\gamma}_{S R}}\right)\right] } \\
\times & {\left[1-\frac{x}{\bar{\gamma}_{S R}}+\mathcal{O}\left(\frac{x}{\bar{\gamma}_{S R}}\right)\right] } \\
= & \left(1-\rho_{S R}^{2}\right)^{N_{t}-1} \frac{x}{\bar{\gamma}_{S R}}+\mathcal{O}\left(\frac{x}{\bar{\gamma}_{S R}}\right)
\end{aligned}
$$

where $\mathcal{O}(x)$ denotes the high-order infinitely small contribu- 850 tions as a function of $x$, and

$$
\begin{aligned}
F_{\gamma_{R^{*} D}}(x) \rightarrow 1 & -\sum_{k=0}^{K_{r}-1}(-1)^{k} \frac{K_{r}}{k+1}\left(\begin{array}{c}
K_{r}-1 \\
k
\end{array}\right) \\
& \times\left[1-\frac{k+1}{k\left(1-\rho_{R D}^{2}\right)+1} \frac{x}{\bar{\gamma}_{R D}}+\mathcal{O}\left(\frac{x}{\bar{\gamma}_{R D}}\right)\right] \\
= & \sum_{k=0}^{K_{r}-1}(-1)^{k}\left(\begin{array}{c}
K_{r}-1 \\
k
\end{array}\right) \frac{K_{r}}{k\left(1-\rho_{R D}^{2}\right)+1} \\
& \times \frac{x}{\bar{\gamma}_{R D}}+\mathcal{O}\left(\frac{x}{\bar{\gamma}_{R D}}\right) .
\end{aligned}
$$

Then, applying the upper bound of the receiver SNR, we may 852 rewrite the COP and the SOP of the TBRS strategy at high 853 SNRs as

$$
\begin{aligned}
P_{\mathrm{co}}^{\mathrm{TBRS}, \infty}= & 1-\left(1-F_{\gamma_{S R^{*}}}\left(\gamma_{t h}^{D}\right)\right)\left(1-F_{\gamma_{R^{*} D}}\left(\gamma_{t h}^{D}\right)\right) \\
= & {\left[\frac{\left(1-\rho_{S R}^{2}\right)^{N_{t}-1}}{\sigma_{S R}^{2}}+\sum_{k=0}^{K_{r}-1}(-1)^{k}\right.} \\
& \left.\times\left(\begin{array}{c}
K_{r}-1 \\
k
\end{array}\right) \frac{K_{r}}{\left[k\left(1-\rho_{R D}^{2}\right)+1\right] \sigma_{R D}^{2}}\right] \frac{2^{2 R_{0}}-1}{\eta}
\end{aligned}
$$

and according to the fact that $\gamma_{R^{*} E}$ is exponentially distributed, 855 we have

$$
\begin{aligned}
1-P_{\mathrm{SO}}^{\mathrm{TBRS}, \infty} & =1-\left(1-F_{\gamma_{S R^{*}}}\left(\gamma_{t h}^{E}\right)\right)\left(1-F_{\gamma_{R^{*} E}}\left(\gamma_{t h}^{E}\right)\right) \\
& =\left[\frac{\left(1-\rho_{S R}^{2}\right)^{N_{t}-1}}{\sigma_{S R}^{2}}+\frac{1}{\sigma_{R E}^{2}}\right] \frac{2^{2\left(R_{0}-R_{s}\right)}-1}{\eta} .
\end{aligned}
$$

Finally, substituting (41) and (42) into the definition of RSR 857 in (16), we can obtain (17). 


\section{PROOF OF LEMMA 1}

861 According to the description of COP and SOP, replacing $862 F_{\gamma_{R^{*} D}}(x)$ and $F_{\gamma_{R^{*} E}}(x)$ by $F_{\xi_{D}}(x)$ and $F_{\xi_{E}}(x)$ in (12) and (14) 863 will involve a mathematically intractable integration of the form

$$
\Upsilon(a, b, \mu, \nu)=\int_{0}^{\infty} \frac{z^{a}}{z+b} \exp \left(-\mu z-\frac{\nu}{z}\right) d z
$$

864 which, to the best of our knowledge, does not have a closed865 form solution. Alternatively, bearing in mind that the preceding 866 integration has a great matter with $\xi_{D}$, we now focus our 867 attention on the approximation of $\xi_{D}$. Based on the PDF 868 results in (23), it may be seen that $\gamma_{J * D}$ obeys an exponential 869 distribution. Then, we can approximate $\hat{\gamma}_{J^{*} D}=\gamma_{J^{*} D}+1$ by 870 the exponential distribution as well, with an average value 871 of $\mathbb{E}\left\{\hat{\gamma}_{J^{*} D}\right\}=\left(\left[\left(K_{r}-1\right)\left(1-\rho_{R D}^{2}\right)+1\right] \bar{\gamma}_{R D}+K_{r}\right) / K_{r}$ by 872 assuming that the AWGN term " 1 " is part of the stochastic 873 mean terms. The approximation based on this method provides 874 a very accurate analysis, and the accuracy of this method is 875 verified by the numerical results of [34]. Thus, the CDF of $876 \hat{\xi}_{D}=\gamma_{R^{*} D} / \hat{\gamma}_{J * D}$ can be derived as

$$
F_{\hat{\xi}_{D}}(x)=\sum_{k=0}^{K_{r}-1}(-1)^{k}\left(\begin{array}{c}
K_{r}-1 \\
k
\end{array}\right) \frac{K_{r}}{k+1} \frac{x}{x+\hat{\varphi}_{k}}
$$

877 where $\hat{\varphi}_{k}=\mathbb{E}\left\{\gamma_{R^{*} D}\right\} \mathbb{E} /\left\{\hat{\gamma}_{J^{*} D}\right\}$.

878 Then, substituting (44) into (11), we have

$$
\begin{aligned}
& F_{\gamma_{D}^{\mathrm{JRJS}}}(x) \\
& \approx \sum_{n=0}^{N_{t}-1} \sum_{k=0}^{K_{r}-1} \sum_{m=0}^{N_{t}-1-n}\left(\begin{array}{c}
N_{t}-1 \\
n
\end{array}\right)\left(\begin{array}{c}
K_{r}-1 \\
k
\end{array}\right)\left(\begin{array}{c}
N_{t}-1-n \\
m
\end{array}\right) \\
& \quad \times \frac{(-1)^{k} K_{r} \rho_{S R}^{2\left(N_{t}-1-n\right)}\left(1-\rho_{S R}^{2}\right)^{n} \varphi_{k} x^{N_{t}-1-n-m} e^{-\frac{x}{\gamma_{S R}}}}{\left(N_{t}-1-n\right) !(k+1) \bar{\gamma}_{S R}^{N_{t}-n}\left(x+\varphi_{k}\right)} \\
& \quad \times \int_{0}^{\infty} \frac{z^{m+1}}{z+\frac{x(x+1)}{x+\varphi_{k}}} \exp \left(-\frac{z}{\bar{\gamma}_{S R}}\right) d z .
\end{aligned}
$$

879 Using [33, eq. (3.383.10)], we can obtain the CDF of $\gamma_{D}^{\text {JRJS }}$ as

$$
\begin{aligned}
F_{\gamma_{D}^{\mathrm{JRJS}}}(x) \approx & 1-\sum_{n=0}^{N_{t}-1} \sum_{k=0}^{K_{r}-1} \sum_{m=0}^{N_{t}-1-n}\left(\begin{array}{c}
N_{t}-1 \\
n
\end{array}\right) \\
& \times\left(\begin{array}{c}
K_{r}-1 \\
k
\end{array}\right)\left(\begin{array}{c}
N_{t}-1-n \\
m
\end{array}\right) \\
& \times \frac{(-1)^{k}\left(K_{r}+1\right) \rho_{S R}^{2\left(N_{t}-1-n\right)}\left(1-\rho_{S R}^{2}\right)^{n}}{\left(N_{t}-1-n\right) !(k+1) \bar{\gamma}_{S R}^{N_{t}-n}} \\
& \times \frac{\Gamma(m+2) \hat{\varphi}_{k} x^{N_{t}-n}(x+1)^{m+1}}{\left(x+\hat{\varphi}_{k}\right)^{m+2}} \\
& \times \exp \left[-\frac{x\left(\hat{\varphi}_{k}-1\right)}{\bar{\gamma}_{S R}\left(x+\hat{\varphi}_{k}\right)}\right] \\
& \times \Gamma\left(-m-1, \frac{x(x+1)}{\bar{\gamma}_{S R}\left(x+\hat{\varphi}_{k}\right)}\right) .
\end{aligned}
$$

As far as the SOP is considered, we exploit the commonly 881 used tight upper bound of $\gamma_{E}^{\mathrm{JRJS}} \geq(1 / 2) \min \left\{\gamma_{S R}, \xi_{E}\right\}$ to 882 calculate it, which may be rewritten as

$$
\begin{aligned}
P_{\mathrm{SO}}^{\mathrm{JRJS}} & \approx \operatorname{Pr}\left\{\frac{1}{2} \min \left\{\gamma_{S R}, \xi_{E}\right\}>\gamma_{t h}^{E}\right\} \\
& =\left[1-F_{\gamma_{S R}}\left(2 \gamma_{t h}^{E}\right)\right]\left[1-F_{\xi_{E}}\left(2 \gamma_{t h}^{E}\right)\right]
\end{aligned}
$$

Substituting (9) and (25) into (47), we obtain $P_{\text {so }}^{\text {JRJS }}$.

\section{APPENDIX C}

\section{PROOF OF LEMMA 3}

According to the definition of the RSCP in (18), we can 887 calculate it by

$$
\begin{aligned}
& P_{R S}^{\mathrm{JRJS}}=\int_{0}^{\infty}\left[1-F_{\xi_{D}}\left(\gamma_{t h}^{D}+\frac{\gamma_{t h}^{D}\left(\gamma_{t h}^{D}+1\right)}{z}\right)\right] \\
& \quad \times F_{\xi_{E}}\left(\gamma_{t h}^{E}+\frac{\gamma_{t h}^{E}\left(\gamma_{t h}^{E}+1\right)}{z+\gamma_{t h}^{D}-\gamma_{t h}^{E}}\right) f_{\gamma_{S R^{*}}}\left(z+\gamma_{t h}^{D}\right) d z
\end{aligned}
$$

To make the integration mathematically tractable, we invoke 889 a simple approximation for $F_{\xi_{E}}(x)$ by treating the AWGN term 890 "1" in $\xi_{E}=\gamma_{R^{*} E} /\left(\gamma_{J^{*} E}+1\right)$ as part of the stochastic mean 891 terms. Hence, we have

$$
F_{\xi_{E}}(x)=\frac{x}{x+\hat{\phi}}
$$

where $\hat{\phi}=\lambda \eta \sigma_{R E}^{2} /\left((1-\lambda) \eta \sigma_{R E}^{2}+1\right)$.

Then, replacing the corresponding CDFs of the second hop 894 with $F_{\hat{\xi}_{D}}(x)$ and $F_{\hat{\xi}_{E}}(x)$ in (26), the integration can be derived as 895

$$
\begin{aligned}
& P_{R S}^{\mathrm{JRJS}} \approx 1-\sum_{n=0}^{N_{t}-1} \sum_{k=0}^{K_{r}-1} \sum_{m=0}^{N_{t}-1-n}(-1)^{k}\left(\begin{array}{c}
N_{t}-1 \\
n
\end{array}\right) \\
& \times\left(\begin{array}{c}
K_{r}-1 \\
k
\end{array}\right)\left(\begin{array}{c}
N_{t}-1-n \\
m
\end{array}\right) \\
& \times \frac{\left(K_{r}+1\right) \rho_{S R}^{2\left(N_{t}-1-n\right)}\left(1-\rho_{S R}^{2}\right)^{n}}{\left(N_{t}-1-n\right) !(k+1) \bar{\gamma}_{S R}^{N_{t}-n}} \\
& \times \frac{\hat{\varphi}_{k}\left(\gamma_{t h}^{D}\right)^{N_{t}-1-n-m}}{\gamma_{t h}^{D}+\hat{\varphi}_{k}} \exp \left(-\frac{\gamma_{t h}^{D}}{\bar{\gamma}_{S R}}-\frac{\gamma_{t h}^{D}}{\omega_{k} \bar{\gamma}_{R D}}\right) \\
& \times \int_{0}^{\infty} e^{\frac{-z}{\gamma_{S R}} z^{m+1}}\left[\frac{1}{z+\theta_{1, k}}-\frac{\hat{\phi}\left(z+\gamma_{t h}^{D}-\gamma_{t h}^{E}\right) e^{\frac{-\gamma_{t h}^{E}}{\gamma_{R E}}}}{\left(\gamma_{t h}^{E}+\hat{\phi}\right)\left(\theta_{1, k}-\theta_{2}\right)}\right. \\
&\left.\times\left(\frac{1}{z+\theta_{2}}-\frac{1}{z+\theta_{1, k}}\right)\right] d z
\end{aligned}
$$

where $\hat{\varphi}_{k}$ and $\hat{\phi}$ are introduced by relying on the similar approx- 896 imation as in Appendix B. Then, using [33, eq. (3.383.10)], we 897 obtain $P_{R \& S}^{J R J S}$. 


\section{REFERENCES}

1] B. Schneier, "Cryptographic design vulnerabilities," Computer, vol. 31, no. 9 , pp. $29-33$, Sep. 1998.

2] A. D. Wyner, "The wire-tap channel," Bell Syst. Techn. J., vol. 54, no. 8, pp. 1355-1387, Oct. 1975.

3] I. Csiszar and J. Korner, "Broadcast channels with confidential messages," IEEE Trans. Inf. Theory, vol. IT-24, no. 3, pp. 339-348, May 1978.

[4] W. K. Harrison, J. Almeida, M. R. Bloch, S. W. McLaughlin, and J. Barros, "Coding for secrecy: An overview of error-control coding techniques for physical-layer security," IEEE Signal Process. Mag., vol. 30, no. 5, pp. 41-50, Sep. 2013.

[5] P. K. Gopala, L. Lai, and H. E. Gamal, "On the secrecy capacity of fading channels," IEEE Trans. Inf. Theory, vol. 54, no. 10, pp. 4687-4698, Oct. 2008.

6] Y. W. P. Hong, P. C. Lan, and C. C. J. Kuo, "Enhancing physical-layer secrecy in multi-antenna wireless systems: An overview of signal processing approaches," IEEE Signal Process. Mag., vol. 30, no. 5, pp. 29-40, Sep. 2013.

[7] R. Bassily et al., "Cooperative security at the physical layer: A summary of recent advances," IEEE Signal Process. Mag., vol. 30, no. 5, pp. 16-28, Sep. 2013.

[8] L. Dong, Z. Han, A. P. Petropulu, and H. V. Poor, "Improving wireless physical layer security via cooperating relays," IEEE Trans. Signal Process., vol. 58, no. 3, pp. 1875-1888, Mar. 2010.

[9] J. Huang and A. L. Swindlehurst, "Cooperative jamming for secure communications in MIMO relay networks," IEEE Trans. Signal Process., vol. 59, no. 10, pp. 4871-4884, Oct. 2011.

0] Y. Zou, X. Wang, and W. Shen, "Optimal relay selection for physical-layer security in cooperative wireless networks," IEEE J. Sel. Areas Commun., vol. 31, no. 10, pp. 2099-2111, Oct. 2013.

11] Y. Zou, X. Wang, W. Shen, and L. Hanzo, "Security versus reliability analysis of opportunistic relaying," IEEE Trans. Veh. Technol., vol. 63, no. 6, pp. 2653-2661, Jul. 2014.

2] I. Krikidis, J. S. Thompson, and S. McLaughlin, "Relay selection for secure cooperative networks with jamming," IEEE Trans. Wireless Commun., vol. 8, no. 10, pp. 5003-5011, Oct. 2009.

3] J. Chen, R. Zhang, L. Song, Z. Han, and B. Jiao, "Joint relay and jammer selection for secure two-way relay networks," IEEE Trans. Inf. Forensic Security, vol. 7, no. 1, pp. 310-320, Feb. 2012.

14] Z. Ding, M. Xu, J. Lu, and F. Liu, "Improving wireless security for bidirectional communication scenarios," IEEE Trans. Veh. Technol., vol. 61, no. 6, pp. 2842-2848, Jul. 2012.

5] C. Wang, H. M. Wang, and X. G. Xia, "Hybrid opportunistic relaying and jamming with power allocation for secure cooperative networks," IEEE Trans. Wireless Commun., vol. 14, no. 2, pp. 589-605, Feb. 2015.

16] H. Deng, H. M. Wang, W. Guo, and W. Wang, "Secrecy transmission with a helper: To relay or to jam," IEEE Trans. Inf. Forensic Security, vol. 10, no. 2, pp. 293-307, Feb. 2015.

7] B. He, X. Zhou, and T. D. Abhayapala, "Wireless physical layer security with imperfect channel state information: A survey," ZTE Commun., vol. 11, no. 3, pp. 11-19, Sep. 2013.

8] A. Mukherjee and A. L. Swindlehurst, "Robust beamforming for security in MIMO wiretap channels with imperfect CSI," IEEE Trans. Signal Process., vol. 59, no. 1, pp. 351-361, Jan. 2011.

19] J. Zhang and M. C. Gursoy, "Relay beamforming strategies for physicallayer security," in Proc. CISS, Princeton, NJ, USA, Mar. 2010, pp. 1-6.

20] M. Bloch, J. Barros, M. R. D. Rodrigues, and S. W. McLaughlin, "Wireless information-theoretic security," IEEE Trans. Inf. Theory, vol. 54, no. 6, pp. 2515-2534, Jun. 2008.

21] X. Zhou, M. R. McKay, B. Maham, and A. Hjorungnes, "Rethinking the secrecy outage formulation: A secure transmission design perspective," IEEE Commun. Lett., vol. 15, no. 3, pp. 302-304, Mar. 2011.

22] J. Hu, Y. Cai, N. Yang, and W. Yang, "A new secure transmission scheme with outdated antenna selection," IEEE Trans. Inf. Forensics Security, to be published.

23] J. Hu, W. Yang, N. Yang, X. Zhou, and Y. Cai, "On-off-based secure transmission design with outdated channel state information," IEEE Trans. Veh. Technol., to be published.

24] N. E. Wu and H. J. Li, "Effect of feedback delay on secure cooperative networks with joint relay and jammer selection," IEEE Wireless Commun. Lett., vol. 2, no. 4, pp. 415-418, Aug. 2013.

25] X. Guan Y. Cai and Y. Yang, "Secure transmission design and performance analysis for cooperation exploring outdated CSI," IEEE Commun. Lett., vol. 18, no. 9, pp. 1637-1640, Sep. 2014.
[26] L. Wang, S. Xu, W. Yang, W. Yang, and Y. Cai, "Security performance 975 of multiple antennas multiple relaying networks with outdated relay 976 selection," in Proc. WCSP, Hefei, China, Oct. 2014, pp. 1-6.

[27] J. Huang and A. L. Swindlehurst, "Buffer-aided relaying for two-hop 978 secure communication," IEEE Trans. Wireless Commun., vol. 14, no. 1, 979 pp. 152-164, Jan. 2015.

[28] S. I. Kim, I. M. Kim, and J. Heo, "Secure transmission for multiuser relay 981 networks," IEEE Trans. Wireless Commun., vol. 14, no. 7, pp. 3724-3737, 982 Jul. 2015.

[29] Y. Ma, D. Zhang, A. Leith, and Z. Wang, "Error performance of transmit 984 beamforming with delayed and limited feedback," IEEE Trans. Wireless 985 Commun., vol. 8, no. 3, pp. 1164-1170, Mar. 2009.

[30] Z. Rezki, A. Khisti, and M. S. Alouini, "Ergodic secret message capac- 987 ity of the wirechannel with finite-rate feedback," IEEE Trans. Wireless 988 Commun., vol. 13, no. 6, pp. 3364-3379, Jun. 2014.

[31] X. Tang, R. Liu, P. Spasojevic, and H. V. Poor, "On the throughput of 990 secure hybrid-ARQ protocols for Gaussian block-fading channels," IEEE 991 Trans. Inf. Theory, vol. 55, no. 4, pp. 1575-1591, Apr. 2009.

[32] H. A. Suraweera, M. Soysa, C. Tellambura, and H. K. Garg, "Performance 993 analysis of partial relay selection with feedback delay," IEEE Signal 994 Process. Lett., vol. 17, no. 6, pp. 531-534, Jun. 2010.

[33] I. S. Gradshteyn and I. M. Ryzhik, Table of Integrals, Series and Products, 996 6th ed. San Diego, CA, USA: Academic, 2000.

[34] S. Kim and J. Heo. "Outage probability of interference-limited amplify- 998 and-forward relaying with partial relay selection," in Proc. IEEE VTC, 999 Yokohama, Japan, May 2011, pp. 1-5.

1000

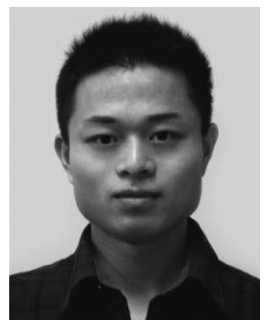

Lei Wang (S'11) received the B.S. degree in elec- 1001 tronics and information engineering from Central 1002 South University, Changsha, China, in 2004 and 1003 the M.S. degree in communications and informa- 1004 tion systems from PLA University of Science and 1005 Technology, Nanjing, China, in 2011. He is currently 1006 working toward the Ph.D. degree in communications 1007 and information systems with PLA University of 1008 Science and Technology.

1009

His current research interests include cooperative 1010 communications, signal processing in communica- 1011 tions, and physical layer security.

1012

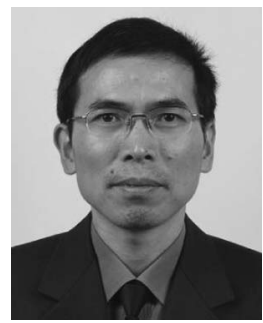

Yueming Cai (M'05-SM'12) received the B.S. 1013 degree in physics from Xiamen University, 1014 Xiamen, China, in 1982 and the M.S. degree in 1015 microelectronics engineering and the Ph.D. degree in 1016 communications and information systems from 1017 Southeast University, Nanjing, China, in 1988 and 1018 1996, respectively.

1019

He is currently with the College of Communica- 1020 AQ5 tions Engineering, PLA University of Science and 1021 Technology, Nanjing, China. His current research 1022 interests include multiple-input-multiple-output sys- 1023 tems, orthogonal frequency-division multiplexing systems, signal processing in 1024 communications, cooperative communications, and wireless sensor networks. 1025 


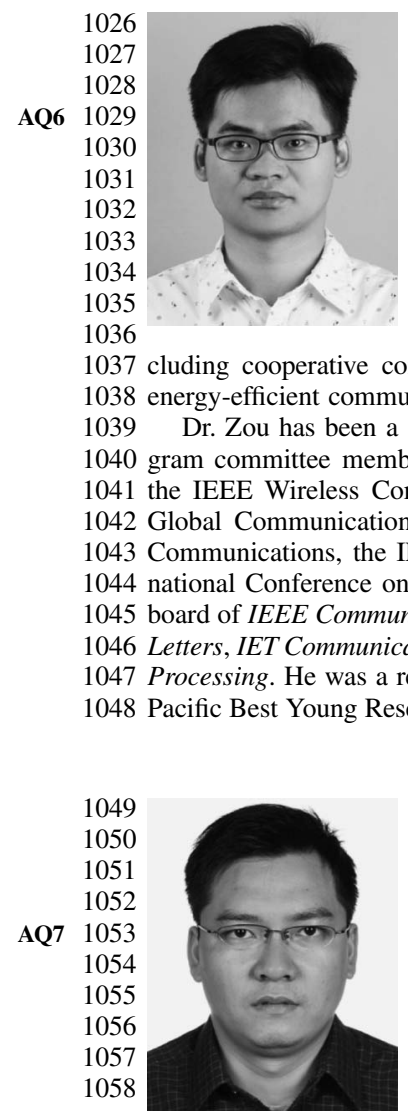

Yulong Zou (SM'13) received the B.Eng. degree in information engineering from Nanjing University of Posts and Telecommunications (NUPT), Nanjing, China, in July 2006; the Ph.D. degree in electrical engineering from Stevens Institute of Technology, Hoboken, NJ, USA, in May 2012; and the Ph.D. degree in signal and information processing from NUPT in July 2012.

$\mathrm{He}$ is currently a Professor with NUPT. His research interests span a wide range of topics in wireless communications and signal processing, inmunications, cognitive radio, wireless security, and

1038 energy-efficient communications. 1040 gram committee member for several IEEE-sponsored conferences, including 1041 the IEEE Wireless Communications and Networking Conference, the IEEE Global Communications Conference, the IEEE International Conference on Comunications, the IEEE Vehicular Technology Conference, and the InterConference on Communications in China. He serves on the editoria Communications Surveys and Tutorials, IEEE Communication was a received the 2014 IEEE Communications Society Asia-

Weiwei Yang (S'08-M'12) received the B.S., M.S. and Ph.D. degrees from PLA University of Science and Technology, Nanjing, China, in 2003, 2006, and 2011, respectively.

$\mathrm{He}$ is currently with the College of Communications Engineering, PLA University of Science and Technology. His research interests are orthogonal frequency-domain multiplexing systems, signal processing in communications, cooperative communications, cognitive networks, and network security.

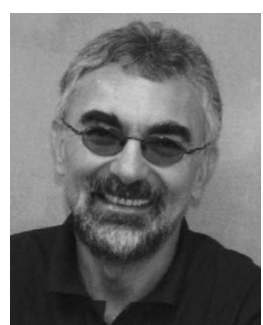

Lajos Hanzo (M'91-SM'92-F'04) received the 1059 M.S. degree in electronics and the Ph.D. de- 1060 gree from the Technical University of Budapest, 1061 Budapest, Hungary, in 1976 and 1983, respectively; 1062 the D.Sc. degree from the University of Southampton, 1063 Southampton, U.K., in 2004; and the "Doctor Honoris 1064 Causa" degree from the Technical University of 1065 Budapest in 2009. 1066

During his 38-year career in telecommunications, 1067 he has held various research and academic posts in 1068 Hungary, Germany, and the U.K. Since 1986, he has 1069 been with the School of Electronics and Computer Science, University of 1070 Southampton, where he holds the Chair in Telecommunications. He is currently 1071 directing an academic research team, working on a range of research projects 1072 in the field of wireless multimedia communications sponsored by industry, the 1073 Engineering and Physical Sciences Research Council (EPSRC), the European 1074 Research Council's Advanced Fellow Grant, and the Royal Society's Wolfson 1075 Research Merit Award. During 2008-2012, he was a Chaired Professor with 1076 Tsinghua University, Beijing, China. He is an enthusiastic supporter of in- 1077 dustrial and academic liaison and offers a range of industrial courses. He 1078 has successfully supervised about 100 Ph.D. students, coauthored 20 John 1079 Wiley/IEEE Press books on mobile radio communications totaling in excess of 1080 10000 pages, and published more than 1400 research entries on IEEE Xplore. 1081

Dr. Hanzo is a Fellow of the Royal Academy of Engineering, the Institution 1082 of Engineering and Technology, and the European Association for Signal 1083 Processing. He is also a Governor of the IEEE Vehicular Technology Society. 1084 During 2008-2012, he was the Editor-in-Chief of IEEE Press. He has served 1085 as the Technical Program Committee Chair and the General Chair of IEEE 1086 conferences, has presented keynote lectures, and has received a number of 1087 distinctions. His published work has more than 20000 citations. Further in- 1088 formation on research in progress and associated publications is available at 1089 http://www-mobile.ecs.soton.ac.uk. 


\section{AUTHOR QUERIES}

\section{AUTHOR PLEASE ANSWER ALL QUERIES}

AQ1 = RV was expanded as "random variable." Please check if appropriate. Otherwise, please make the necessary changes.

AQ2 = Equations (29) and (30) are missing in the document. Please check.

AQ3 = Please provide publication update in Ref [22].

AQ4 = Please provide publication update in Ref [23].

AQ5 = Current affiliation of author Yueming Cai was provided as captured from the first footnote. Please check if appropriate. Otherwise, please make the necessary changes.

AQ6 $=$ Please confirm that Dr. Zou has received two Ph.D. degrees.

AQ7 = Current affiliation of author Weiwei Yang was provided as captured from the first footnote. Please check if appropriate. Otherwise, please make the necessary changes.

END OF ALL QUERIES 